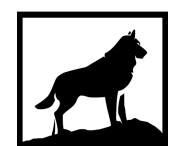

Michigan Technological

1 8 8 5 University
Michigan Technological University

Digital Commons @ Michigan Tech

\title{
INTEGRATION OF ROBOTIC AND ELECTRO-PNEUMATIC SYSTEMS USING ADVANCED CONTROL AND COMMUNICATION SCHEMES
}

Chinmay Kondekar

Michigan Technological University, crkondek@mtu.edu

Copyright 2021 Chinmay Kondekar

\section{Recommended Citation}

Kondekar, Chinmay, "INTEGRATION OF ROBOTIC AND ELECTRO-PNEUMATIC SYSTEMS USING ADVANCED CONTROL AND COMMUNICATION SCHEMES", Open Access Master's Thesis, Michigan Technological University, 2021.

https://doi.org/10.37099/mtu.dc.etdr/1218

Follow this and additional works at: https://digitalcommons.mtu.edu/etdr

Part of the Controls and Control Theory Commons, Digital Communications and Networking Commons, Electrical and Electronics Commons, Engineering Education Commons, Hardware Systems Commons, Other Electrical and Computer Engineering Commons, Robotics Commons, and the Systems and Communications Commons 
INTEGRATION OF ROBOTIC AND ELECTRO-PNEUMATIC SYSTEMS USING ADVANCED CONTROL AND COMMUNICATION SCHEMES

\author{
By \\ Chinmay R Kondekar
}

\begin{abstract}
A THESIS
Submitted in partial fulfillment of the requirements for the degree of MASTER OF SCIENCE

In Electrical Engineering
\end{abstract}

MICHIGAN TECHNOLOGICAL UNIVERSITY

2021

(C) 2021 Chinmay R Kondekar 
This thesis has been approved in partial fulfillment of the requirements for the Degree of MASTER OF SCIENCE in Electrical Engineering.

Department of Electrical and Computer Engineering

$\begin{array}{cc}\text { Thesis Advisor: } & \text { Aleksandr Sergeyev } \\ \text { Committee Member: } & \text { John E. Pakkala } \\ \text { Committee Member: } & \text { David M. Labyak } \\ \text { Committee Member: } & \text { John L. Irwin } \\ \text { Department Chair: } & \text { Glen E. Archer }\end{array}$




\section{Table of Contents}

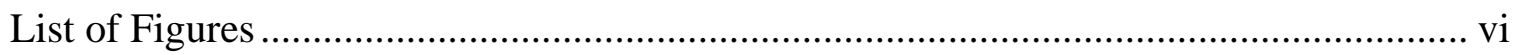

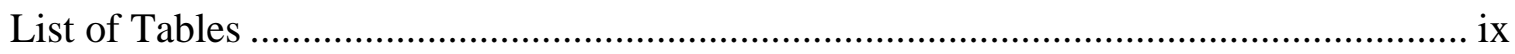

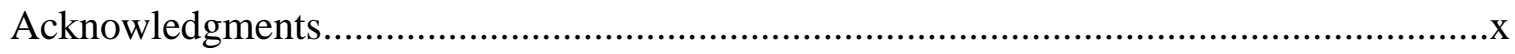

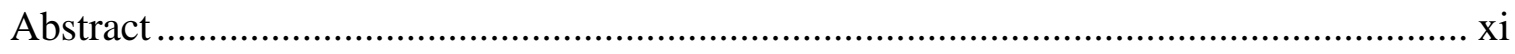

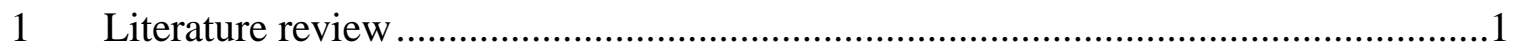

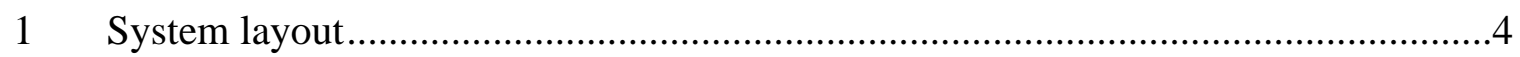

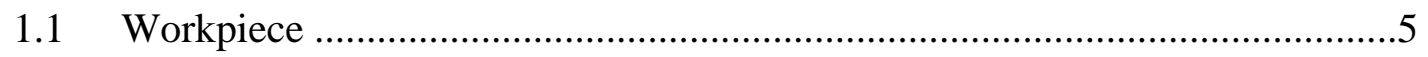

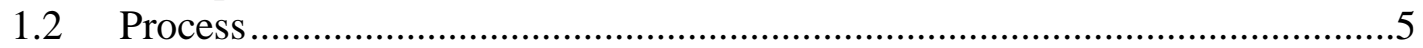

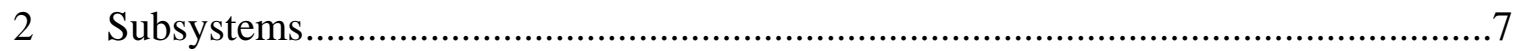

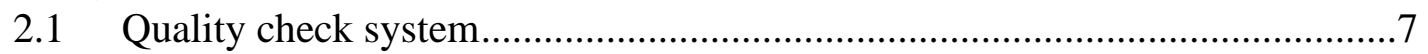

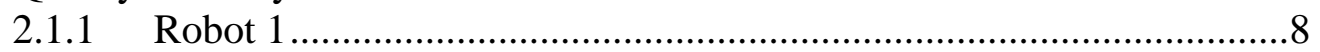

2.1.2 Robot 1 end of the arm tooling ...................................................

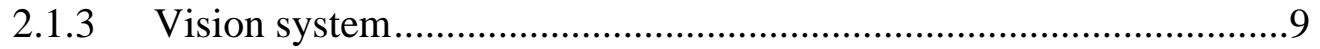

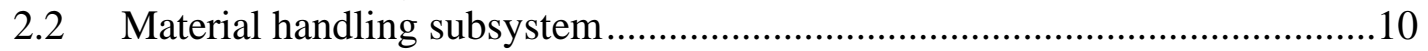

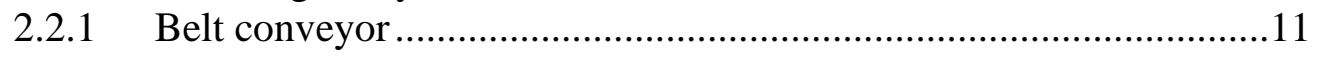

2.2.2 Inductive proximity sensors ..........................................................11

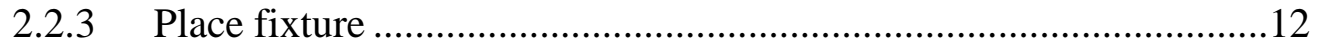

2.2.4 Photoelectric sensor .................................................................

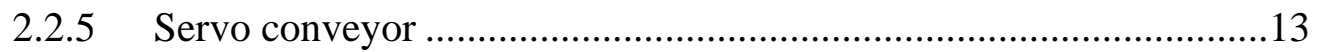

2.2.6 Donald Engineering station one ......................................................13

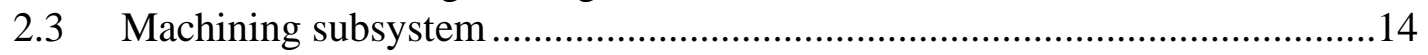

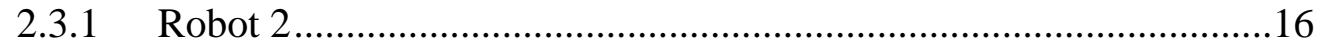

2.3.2 Robot-2 End Of the Arm Tooling .................................................16

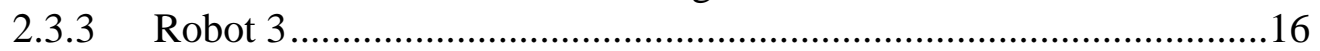

2.3.4 Robot-3 End Of the Arm Tooling .................................................17

2.3.5 Dremel Tooling .............................................................................18

2.3.6 Gripper tooling .......................................................................19

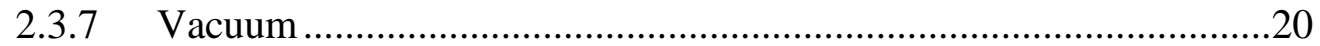

2.4 Control and communication subsystem ……………...............................20

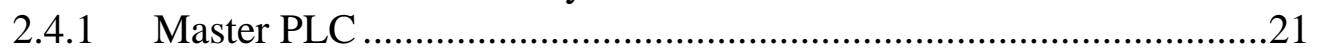

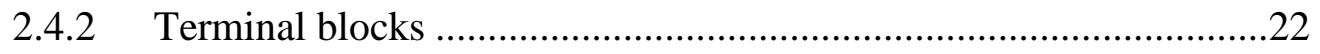

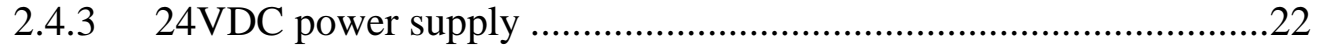

2.4.4 Ethernet switch..........................................................................22

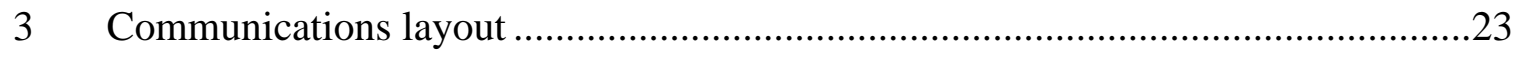

3.1 Network topology and IP addresses .........................................................24

3.2 Configuring EthernetIP on the Robots ......................................................24

3.3 Configuring EthernetIP on the master PLC …………................................26 


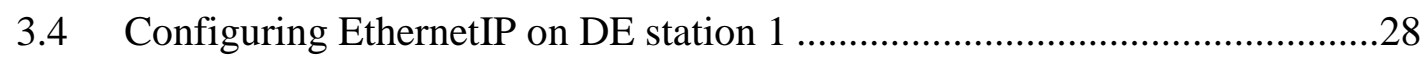

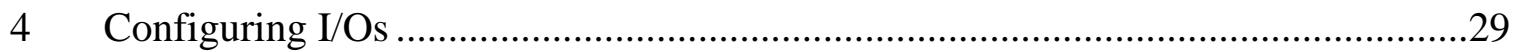

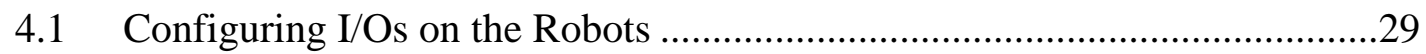

4.1.1 Setting EthernetIP I/Os on the robots .........................................29

4.1.2 Setting hardwired I/Os on the robots ............................................

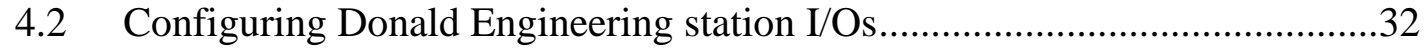

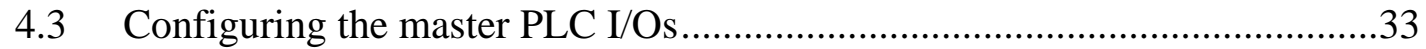

4.3.1 Setting EthernetIP I/Os on the master PLC .................................33

4.3.2 Setting hardwired I/Os on the master PLC ..................................35

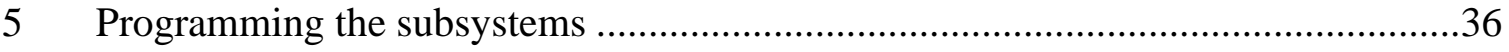

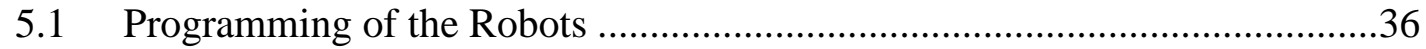

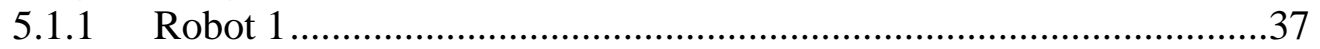

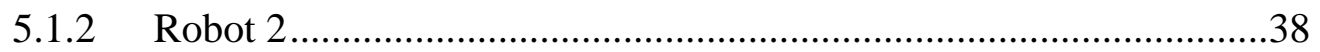

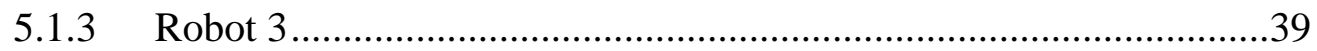

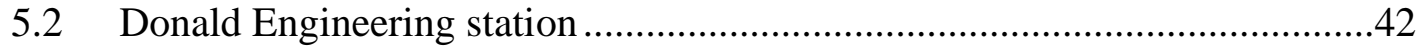

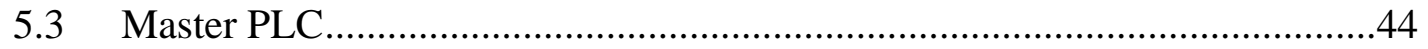

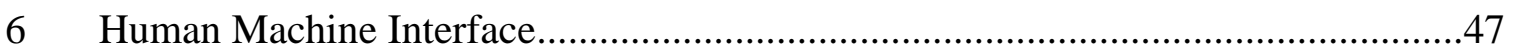

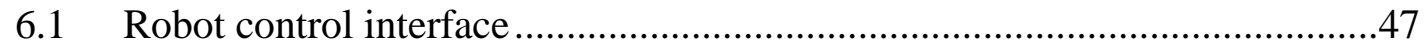

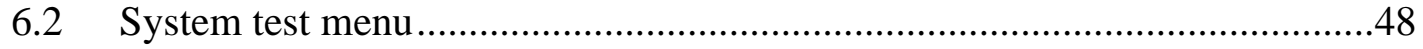

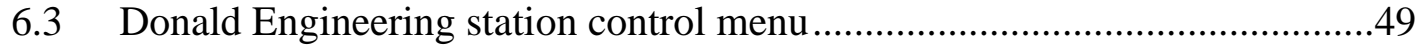

6.4 Process control menu........................................................................50

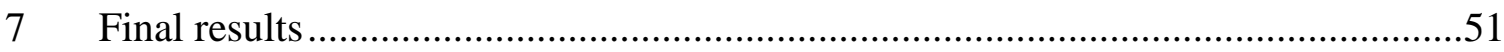

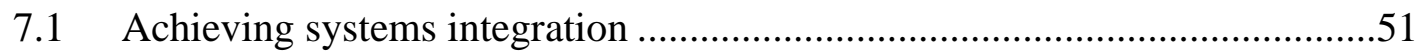

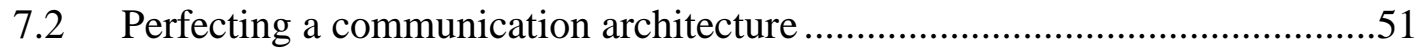

7.3 Standardization and reconfigurability ...................................................52

7.4 Validating the accuracy of robotic machining .........................................52

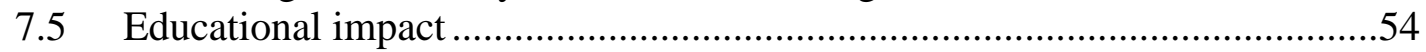

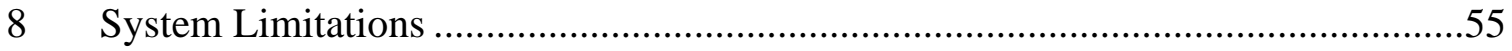

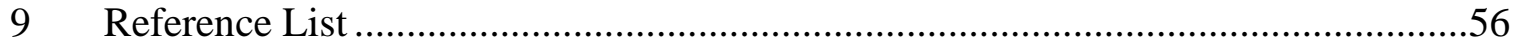

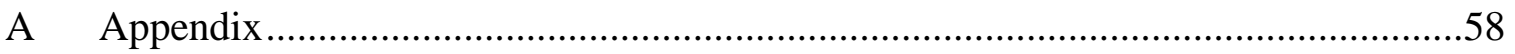

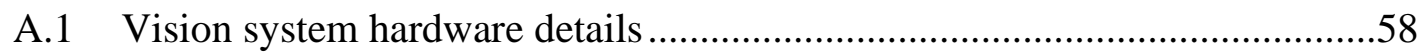

A.2 Donald Engineering station PLC hardware details ..................................58

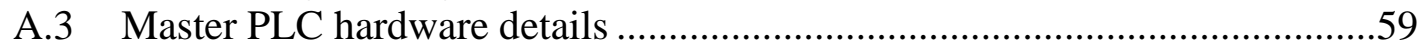

A.4 Robot 2 end of the arm tooling details ....................................................59

A.5 Quick tool changer hardware details .......................................................60

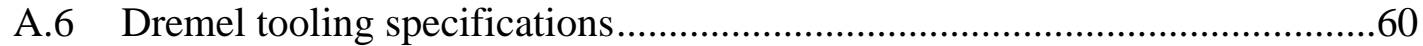

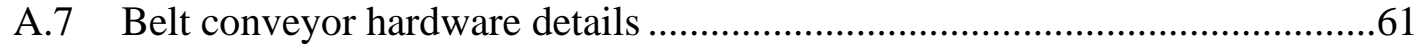

A.8 Photoelectric sensors specifications ........................................................61

A.9 Servo conveyor hardware details ............................................................62 
A.10 Inductive proximity sensor hardware details.........................................62 


\section{List of Figures}

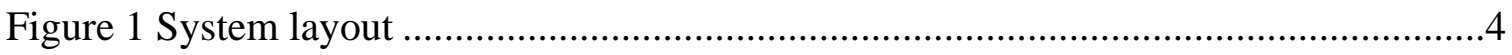

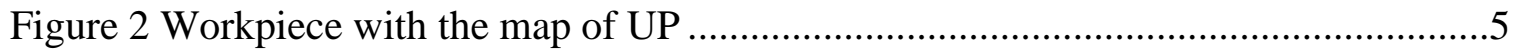

Figure 3 Workpiece with DE and MTU logos................................................................

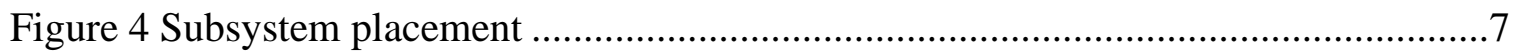

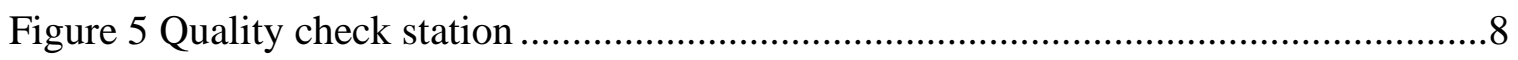

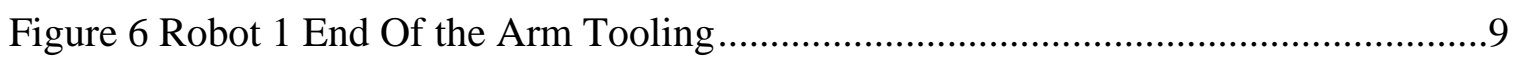

Figure 7 [1] SONY XC-56 camera [2] LED spotlight..................................................

Figure 8 Material handling subsystem operation........................................................10

Figure 9 Belt conveyor, driver, and motor ...................................................................11

Figure 10 Inductive proximity sensors ……………….............................................11

Figure 11 Place fixture ................................................................................12

Figure 12 Photoelectric emitter and receiver ...........................................................12

Figure 13 Servo conveyor and servo driver .................................................................13

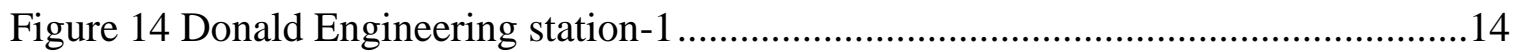

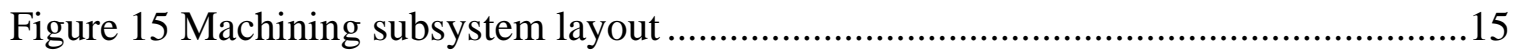

Figure 16 Sequence of machining operation ………………........................................15

Figure 17 Robot-2 End Of the Arm Tooling …………….........................................16

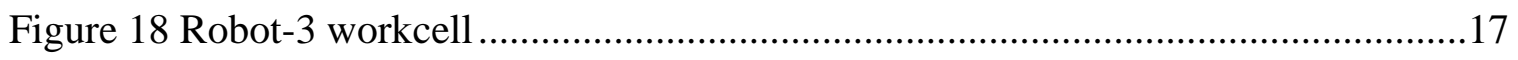

Figure 19 [1] Electronic manifold [2] Relays ………………………………………....18

Figure 20 Dremel tooling .........................................................................................18

Figure 21 Gripper tooling ................................................................................19

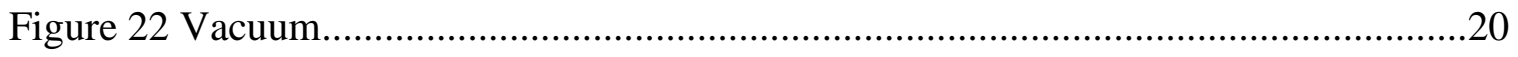

Figure 23 Control and communication subsystem......................................................20

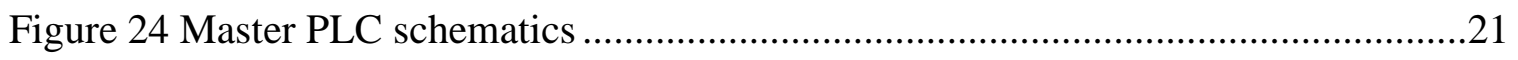

Figure 25 Ethernet switch ...................................................................................22

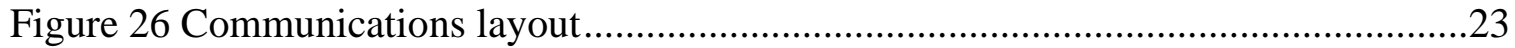

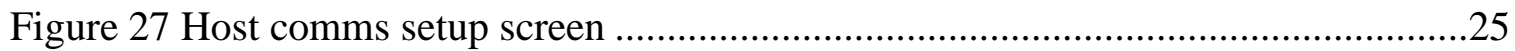

Figure 28 Setting the Laptop IP address ..................................................................26

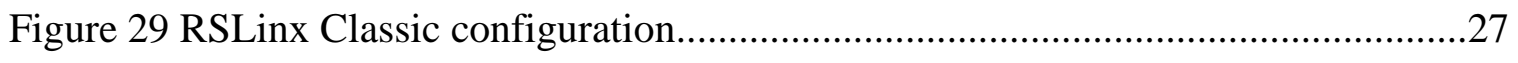

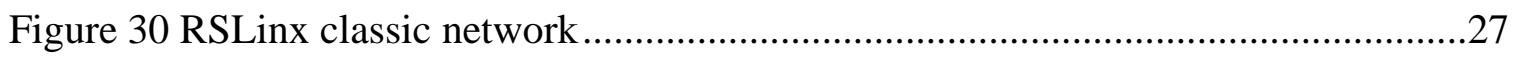

Figure 31 Unitronics PLC IP address configuration...................................................28 


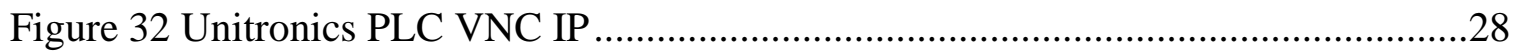

Figure 33 I/O configuration menu on FANUC robots ................................................29

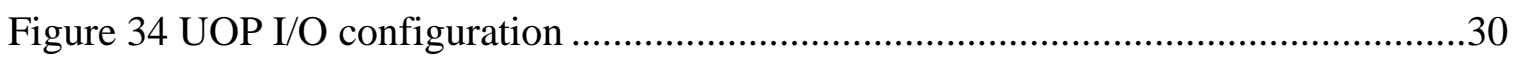

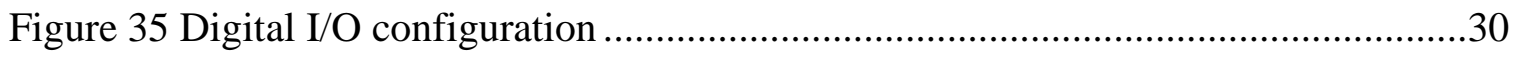

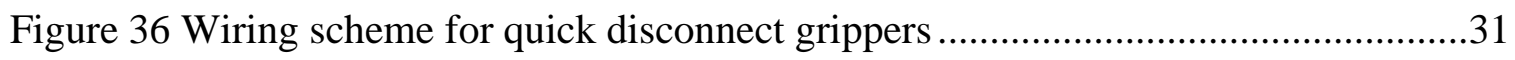

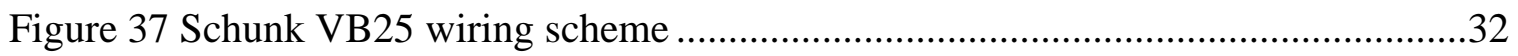

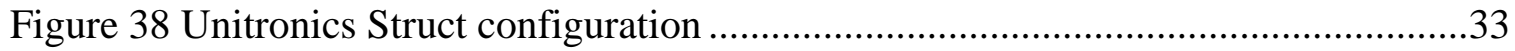

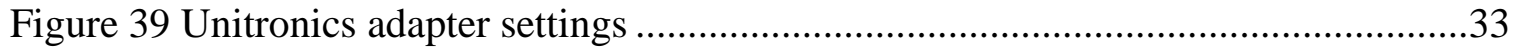

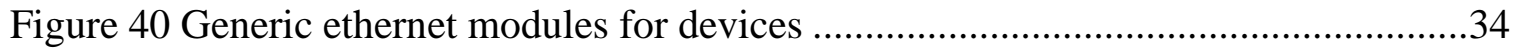

Figure 41 Generic ethernet module in the device tree .................................................34

Figure 42 Master PLC hardwired I/O specification.....................................................35

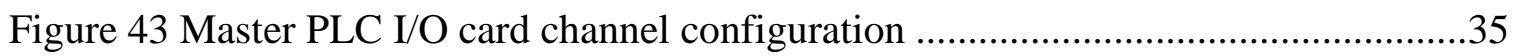

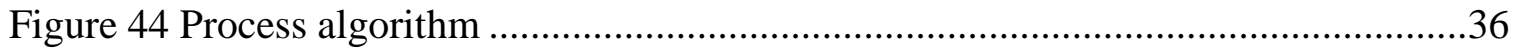

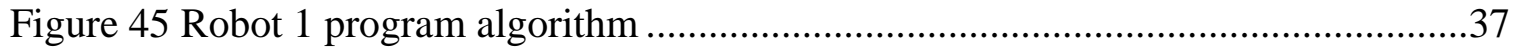

Figure 46 FANUC Roboguide showing Robot 2s position ..........................................38

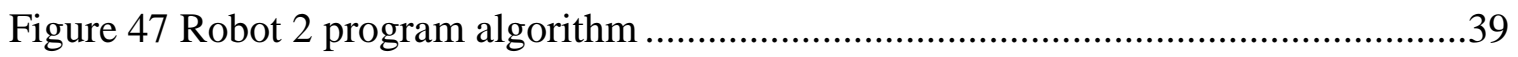

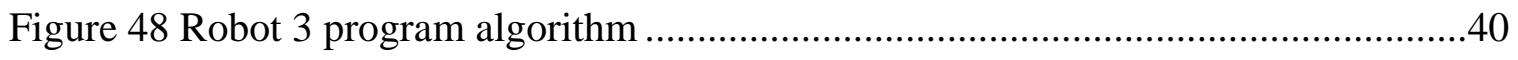

Figure 49 CAD to path on Roboguide ................................................................ 40

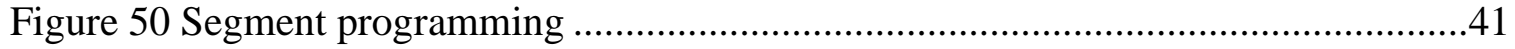

Figure 51 Tool orientation for Roboguide ...........................................................41

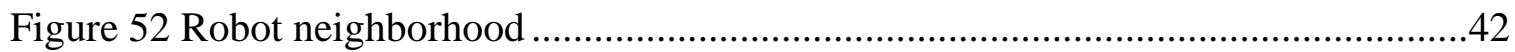

Figure 53 Output commands from Unitronics PLC to the master PLC..........................43

Figure 54 Actuator control logic on the Unitronics PLC ............................................43

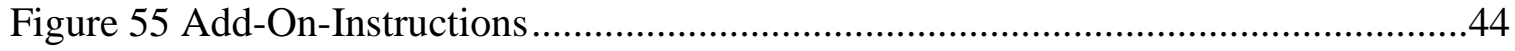

Figure 56 Robot_ops Add-On-instruction called in the program for Robot 1.................44

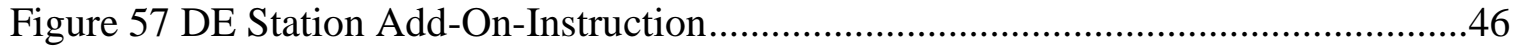

Figure 58 Algorithm for Donald Engineering station machining operations ..................46

Figure 59 FactoryTalk View Studio interface ........................................................47

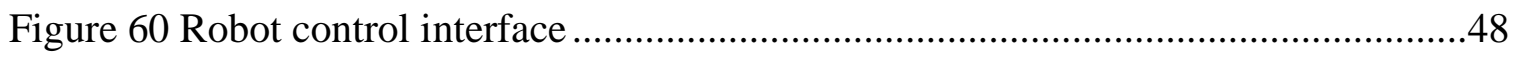

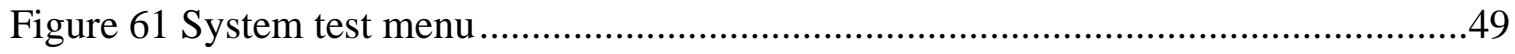

Figure 62 Donald Engineering station control menu ...............................................49

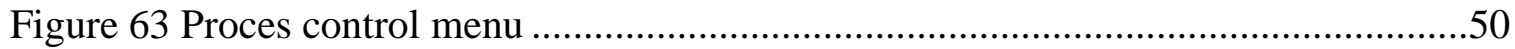




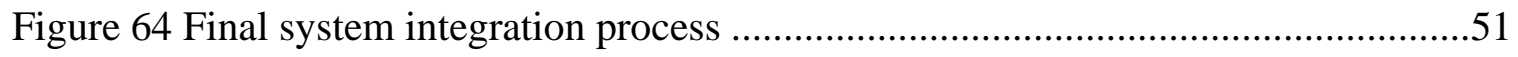

Figure 65 Standardization of robot control interface and algorithm.............................52

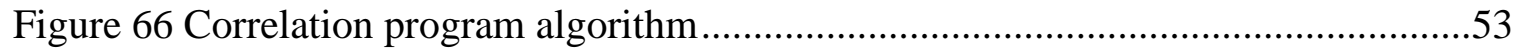

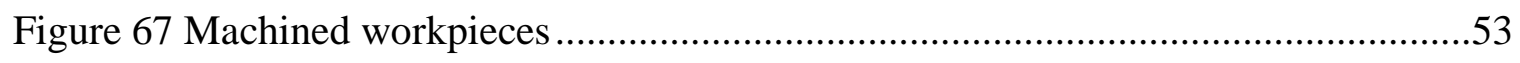

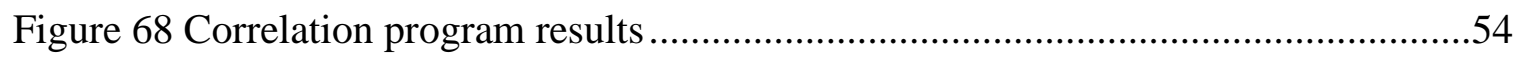




\section{List of Tables}

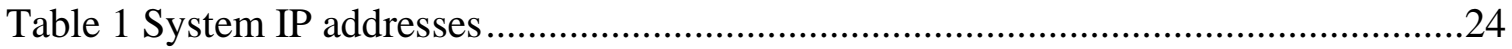

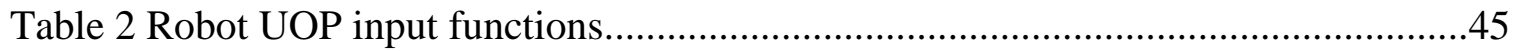

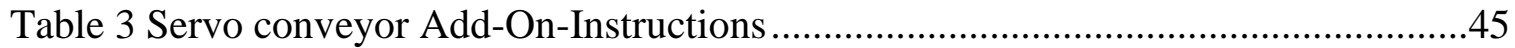

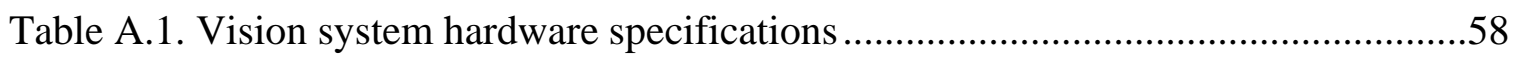

Table A.2. Donald Engineering station PLC hardware specifications ...........................58

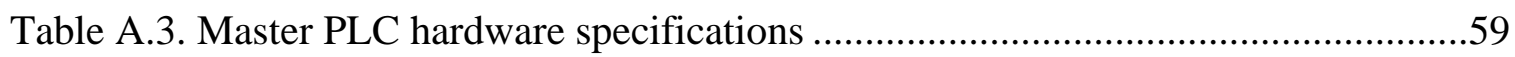

Table A.4. Robot 2 end of the arm tooling gripper specifications ................................59

Table A.5. Quick tool changer hardware specifications .............................................60

Table A.6. Dremel tooling hardware specifications .................................................60

Table A.7. Belt conveyor specifications ............................................................61

Table A.8. Photoelectric sensor specifications ........................................................61

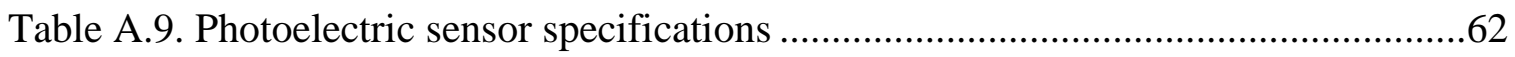

Table A.10. Inductive proximity sensor specifications .............................................62 


\section{Acknowledgments}

First and foremost, I would like to thank my advisor, Dr. Aleksandr Sergeyev, for guiding and supporting me during my graduate studies. His drive towards continuous improvement has been my motivation to achieve my research project goals.

I would also like to express my gratitude to Mark Gauthier, President, Donald Engineering company, for supporting engineering education at Michigan Technological University. This project would not have been possible without his generous donation of industrial equipment and his expertise in electro-pneumatic systems.

I want to thank Professor David M. Labyak for his guidance in machining and material selection. The hardware customization in the project would not have been possible without Mr. Nick Hendrickson, who 3D printed parts for the project and processed the raw workpiece. I also had the pleasure of working with my fellow graduate students Gaurav Pathak and Pratik Korgaonkar, who helped in developing the CAD models for the custom gripper fingers in the project.

I would also like to acknowledge FANUC tech support, the forum members of plctalk.net, and robot-forum.com for providing tech support during the project's development.

Finally, I want to express my profound gratitude to my family and friends for supporting and encouraging me to achieve my dreams and goals. 


\section{Abstract}

Modern industrial automation systems are designed by interconnecting various subsystems which work together to perform a process. The thesis project aims to integrate fragmented subsystems into a flexible and reconfigurable system through advanced communication protocols and perform a process to demonstrate the effectiveness of interconnected systems.

The system consists of three six-axis robots, one electro-pneumatic robot, and two conveyors connected using EthernetIP communication and hardwired connections. The interconnected system works together to perform machining of a workpiece using advanced control methods of CAD to robot path generation, central control through a PLC, and process control through HMI.

Standardized programming blocks and HMI interfaces were developed to make the system highly reconfigurable and flexible for any future projects. The knowledge gained from the project is used to create lab manuals to educate students about communication and control methods for systems integration. 


\section{Literature review}

Modern industrial processes rely on sensor technology to carry out precise functions and involve technologies from touchscreen tablets and phones to robotic assembly machines. Advanced manufacturing incorporates complicated electromechanical systems with cutting-edge control systems to increase production quality and throughputs. Today's manufacturing is highly dependent on automation to optimize the workforce by relieving repetitive and dull tasks while enabling creative work, research, and development. While the automation systems can significantly vary in complexity of controls, sensors, and actuators, the critical parts of the automation infrastructure are the PLCs (Programmable Logic Controllers) and industrial robots. Once integrated with field devices like pneumatic systems, motion control systems, and sensors, they serve as powerful platforms for complete automated systems with reconfigurable functionality

In the proposed automation system, a three-robot workcell performs a machining operation. Depending on the requirements, robotically equipped automation solutions can utilize one robot or multiple. In many cases, multi-robot automation solutions use a PLC as a master controller designated to handle the safety interlocks, program execution of the robot, and Human Machine Interface (HMI). Frequently, the subsystems are connected using EthernetIP communication protocol in the "Adapter - Scanner" configuration, eliminating the need for wired connections between remotely located components. The PLC is the scanner device initiating connection requests, and the robot is an adapter device, providing data to scanners. The process of combining the various subsystem under one control and ensuring synchronized operation is called systems integration.

This literature review aims to find the extent of available information and the level of research done on this topic. This literature review is split into three sections.

\section{Role of PLC's and need of an HMI system}

A PLC is an industrial computer used in manufacturing to control the process based on the inputs and outputs from sensors. A large-scale production facility uses a distributed control system to exchange data. Still, there is a need to acquire production-related data from the factory floor and present it to the production staff. The data collection is done by connecting the PLCs with databases through various communication protocols such as EthernetIP, the workhorse of industrial automation communications. Anthony, Mahato, Sharma, and Chitranshi [1] have reinforced this claim and proposed architecture for such an EthernetIP based system. The authors have used a Linux-based server to collect data and a python program to analyze the collected data, process the information, and present it on a graphical user interface. The system architecture is explained clearly with the help of diagrams and is easy to understand. The paper concludes with the potential applications in the industry.

The system in the proposed consists of many subsystems stitched together. Jeong, Ji, Jung, and Koo [2] offer a software structure having a PLC-controlled robot in which the PLC acts as a master controller. The authors examine the languages presented under IEC61131 and designed the control philosophy according to it. The software structure consists of 
interconnections between different subsystems, and the control algorithm is deployed on a laptop to control three stepper motors.

Any industrial control system requires human supervision and control, achieved through a Supervisory, Control and Data Acquisition System (SCADA) and Human Machine Interface (HMI). Brandin [3] breaks down the individual components of a manufacturing environment by analyzing Discrete Event Systems (DES), an event-driven system and not time-driven systems. Event-driven systems are much better and safer systems to integrate different units as timers may drift after a while, but event-based feedback doesn't have this problem. The authors provide a detailed breakdown of various Supervisory Control and Data Acquisition (SCADA) system elements with a description of the development cycle. The authors also elaborated on the description of programming strategies available in a PLC and the techniques for deploying them. Using these strategies, the SCADA and the PLC have been integrated into an experimental setup to coordinate a robot, conveyor, and vision system. The paper [3] demonstrates methods to integrate different systems and the control methods through an HMI interface and reinforces the claim that an event-based system is better than a time-based system.

Manufacturing applications can be diverse, as stated by Malakanagouda, Harisha [4], who have taken the concept of industrial automation and applied it to automating a brake actuator test rig. The authors have stated the methodology to design the system by explaining the CAD modeling, pneumatic circuit design, ladder programming, and SCADA system design. The results show an exact comparison of execution times in automatic and manual operations. The source establishes the advantage of automated systems and enforces the fact of improvement in the system efficiency.

\section{Challenges and limitations of industrial robots -}

The proposed project aims to integrate a highly flexible and customizable system that can perform any task by reconfiguring the system. The application for the proposed project is machining a CNC foam block by using two six-axis robots. Usually, robots have lower accuracy than dedicated CNCs due to differences in their drive system. T Kubela [5] analyzes why robots have lower accuracy than $\mathrm{CNCs}$ for machining applications by building a system having a six-axis Kuka robot with a milling spindle and Delcam PowerMILL for programming. The error in the machined part's precision is calculated by subtracting the measured path from the predefined path. The calculated error is compensated by implementing a closed-loop control system with a Proportional, Integral, and Derivative (PID) control algorithm to correct the servo governor.

The industrial robotic arm is a manipulator that can repeatedly perform a task with accuracy in microns. However, if the robot is incorrectly calibrated, any errors get compounded due to forward kinematics. Lee, Park, Shin, and Woo [6] investigate the application of Denavit - Hatenberg (DH) parameters using forward kinematics to calibrate industrial robots. The experimental setup consists of a six-axis robot and a laser tracker to monitor the robot's motion and calculate the offset. Once the offset is calculated, the tooling position is computed using DH parameters, and the robot is repositioned accordingly. 
In certain situations, a robot might present infinite solutions to move from one point to another. This event is referred to as a singularity, which should be avoided while programming the robot. Abderrahmane, Djuric, Chen, and Yeh [7] have studied singularities for a FANUC LRMate 200iC robot by analyzing the linkage lengths and joint angles correlating to the Denavit - Hartenberg parameters. The robot's Jacobian matrix is calculated to find the velocities, and the joints are decoupled by using inverse kinematics to simplify the calculations. The author introduces different joint angle combinations triggering singularity conditions in FANUC Robot and provides a visual interpretation of these occurrences using Matlab environment.

Robots have existed since the 1960s but have grown into advanced multi-purpose systems capable of doing repeatable tasks and with high accuracy. PLC and robots are significant parts of the proposed project's subsystems being coordinated over EthernetIP. Moon, Lee, and Kim in [8] propose a mathematical model of a two-robot cell with simultaneous, coordinated, and overlapping motions. A logic model shows the order of tasks to be completed, while a time-based model performance model demonstrates the execution of tasks as a vector model per unit time. The authors also have a fault handling algorithm that restarts the execution if any fault is detected.

\section{Role of EtherNetIP Communications in industrial automation -}

With modern communication protocols such as EthernetIP, fast transmission speeds allow optimal data connection, analysis, and transmission of I/O signals. The interconnectivity of subsystems with EthernetIP communication capabilities is the basis for the Industrial Internet of Things (IIoT), also known as Industry 4.0, which promotes continuous improvement through data collection and analysis. Bassi [9] analyses interconnectivity and data collection aspects of IIoT. Manufacturing execution systems (MES) take advantage of the interconnectivity by collecting the data from PLCs on the factory floor simultaneously tracking individual components on the production line from raw material to finished parts. These data collected by the MES system is analyzed and is helps in production planning.

\section{Future work and research -}

The findings indicate that EthernetIP based systems are much better than hardwired systems as it reduces the clutter and enables a more significant size of data to be exchanged.

The findings also indicate that industrial automation technology is application-driven, where the system is designed to perform a single task, and flexibility is not the primary concern. The proposed system's design philosophy is flexibility, where the master PLC acts as the task manager for the robotic subsystems while communicating over EthernetIP. The robots in the proposed system have additional flexibility due to quick tool changers that enable the rapid exchange of the end-effectors without stopping the production process. 


\section{System layout}

The proposed project aims to integrate subsystems into a system and demonstrate its capabilities by executing a process. The subsystems individually are capable of performing singular tasks without much flexibility. The flexibility and useability of the system are increased by connecting these subsystems to enable signal handshaking between them.

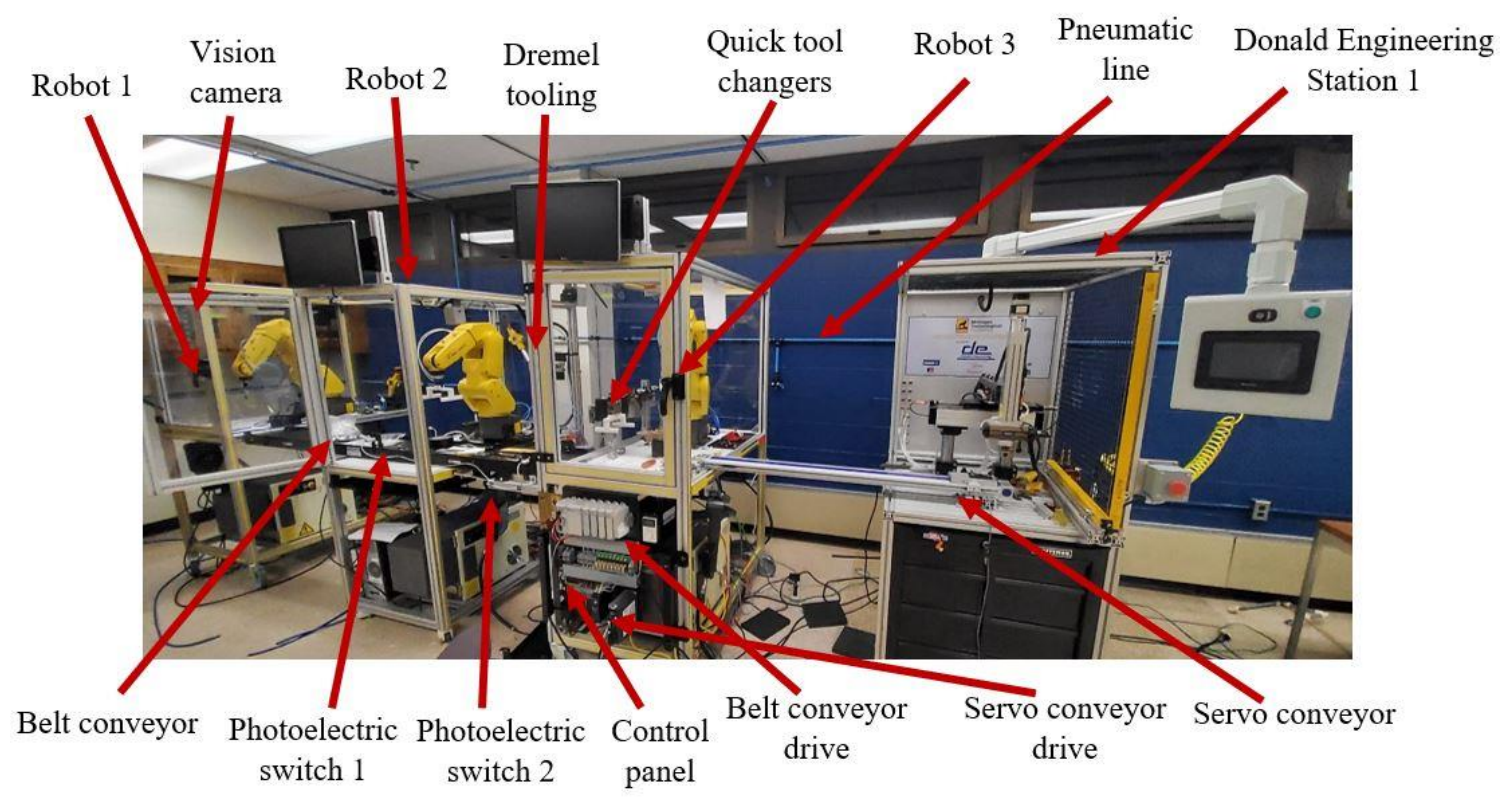

Figure 1 System layout

Figure 1 shows the layout of the system. The system consists of three robots labeled Robot 1, Robot 2, and Robot 3 from left to right. The robots are six-axis FANUC LRMate 200iC with five kilograms of payload, equipped with the vision system, and mounted on a FANUC educational training cart.

A belt conveyor connects three robots and transfers the workpiece between the robotic stations. Photoelectric Switches 1 and 2 detect the presence of a workpiece.

In the proposed process, Robot 3 performs various operations requiring different tooling. Robot 3 has access to quick tool changers and Dremel tooling, which enable tool changing without interrupting the process.

The servo conveyor transfers the workpiece to Donald Engineering station one after being processed by Robot 3. The Donald Engineering station one is a four-axis electro-pneumatic robot. It picks up the workpiece from the servo conveyor and drops it off on a chute to be picked by the operator.

The control panel houses PLC, I/O cards, power supply, terminal busses, and relays mounted on DIN rails. The panel also has mounting points for the belt conveyor and the servo conveyor drivers. 


\subsection{Workpiece}

The operator can select two different patterns on the HMI for the machining operation. The first pattern, shown in Figure 2, is the outline of the Upper Peninsula of Michigan.

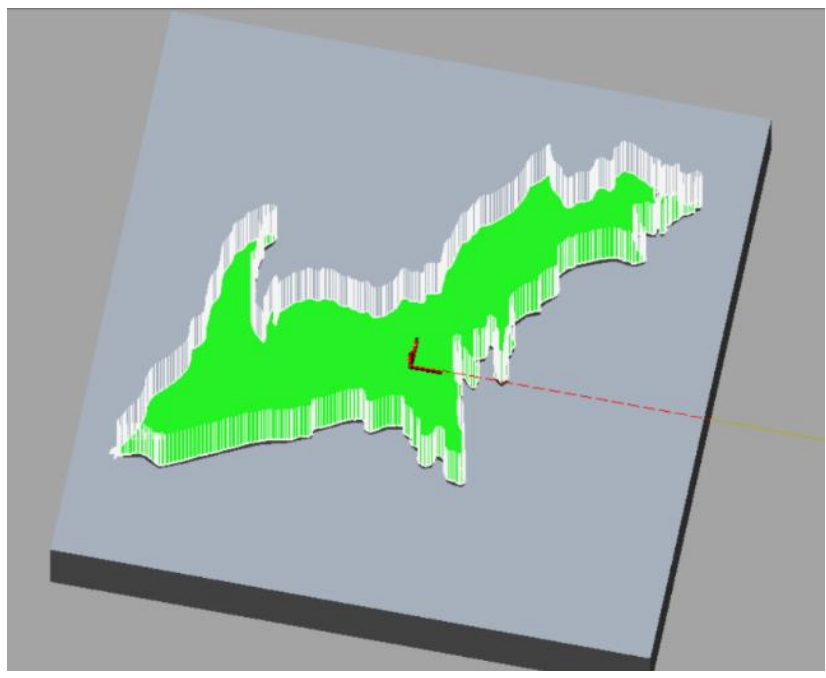

Figure 2 Workpiece with the map of UP

The second pattern is an outline of a robot and logos of Donald Engineering and Michigan Tech, shown in Figure 3. Both designs are machined on a piece of CNC foam measuring $150 \mathrm{~mm} \times 120 \mathrm{~mm}$ X $20 \mathrm{~mm}$ with a density of $81 \mathrm{~b} / \mathrm{ft} 3$.

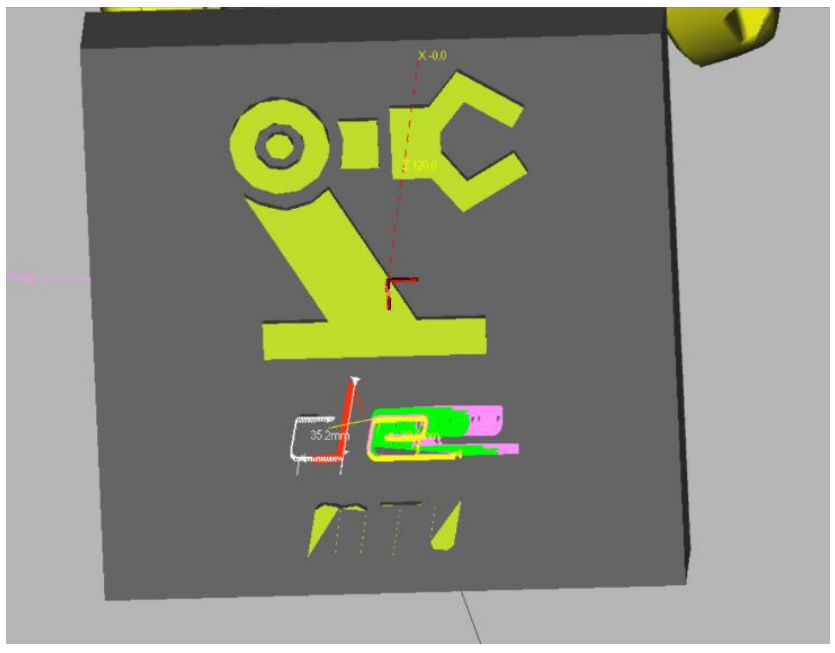

Figure 3 Workpiece with DE and MTU logos

\subsection{Process}

As shown in Figure 1, the process flow is as follows: 
1. The raw workpiece is placed manually in front of Robot 1. The operator selects either map of UP or DE and MTU logos patterns on HMI

2. The vision system on Robot 1 detects the presence of the workpiece. If the workpiece is present and the dimensions match, it is placed on the belt conveyor. Otherwise, the workpiece gets rejected

3. The workpiece moves on the belt conveyor until detected by the photoelectric switch 1

4. Robot 2 picks up the workpiece from the belt conveyor and positions it in front of Robot 3

5. Robot 3 picks the Dremel tooling to machine the previously selected pattern on the workpiece

6. Once the machining operation is complete, Robot 2 places the workpiece on the belt conveyor, and it moves until being detected by the photoelectric switch 2

7. Robot 3 changes the Dremel tooling to the gripper, picks the workpiece from the belt conveyor, and places it on the servo conveyor

8. DE station collects the workpiece from the servo conveyor and delivers it to the operator 


\section{Subsystems}

The proposed system has four major subsystems quality check, material handling, machining, control and communication. The subsystems are interconnected using various communication protocols, enabling them to exchange data to coordinate process execution. The master PLC handles the coordination of the entire system and acts as a master controller.

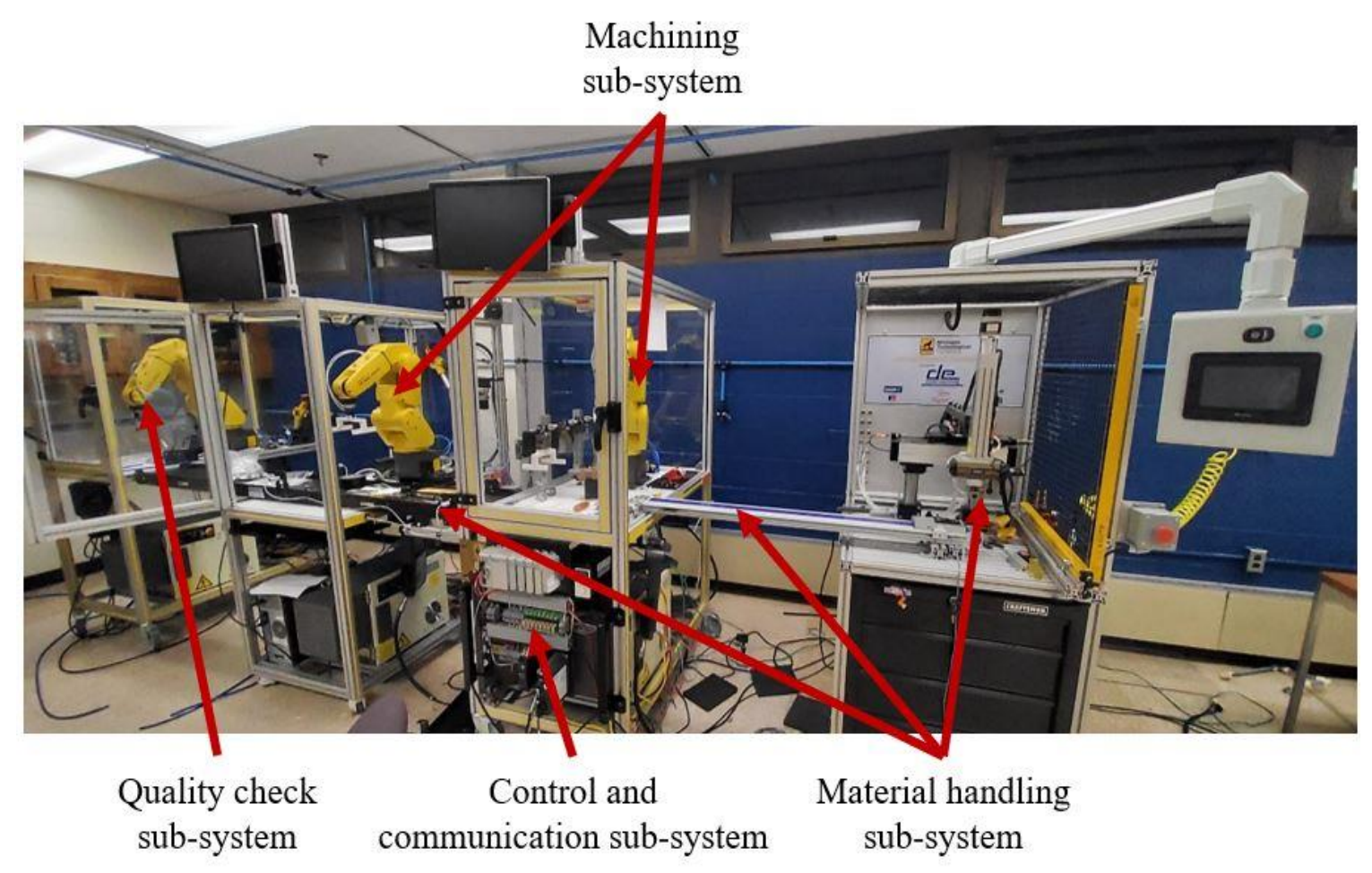

Figure 4 Subsystem placement

\subsection{Quality check system}

The quality check subsystem, shown in Figure 5, is the first part of the process and is designated for checking the dimensions of the raw workpiece. The workpiece gets machined if it matches the required dimensions or rejected if the dimensions are out of tolerance.

The system has a FANUC educational training cart with a six-axis robot and a camera for machine vision integrated into the robot controller.

The robot uses a vacuum gripping end effector to adapt to different shapes and sizes of workpieces. 


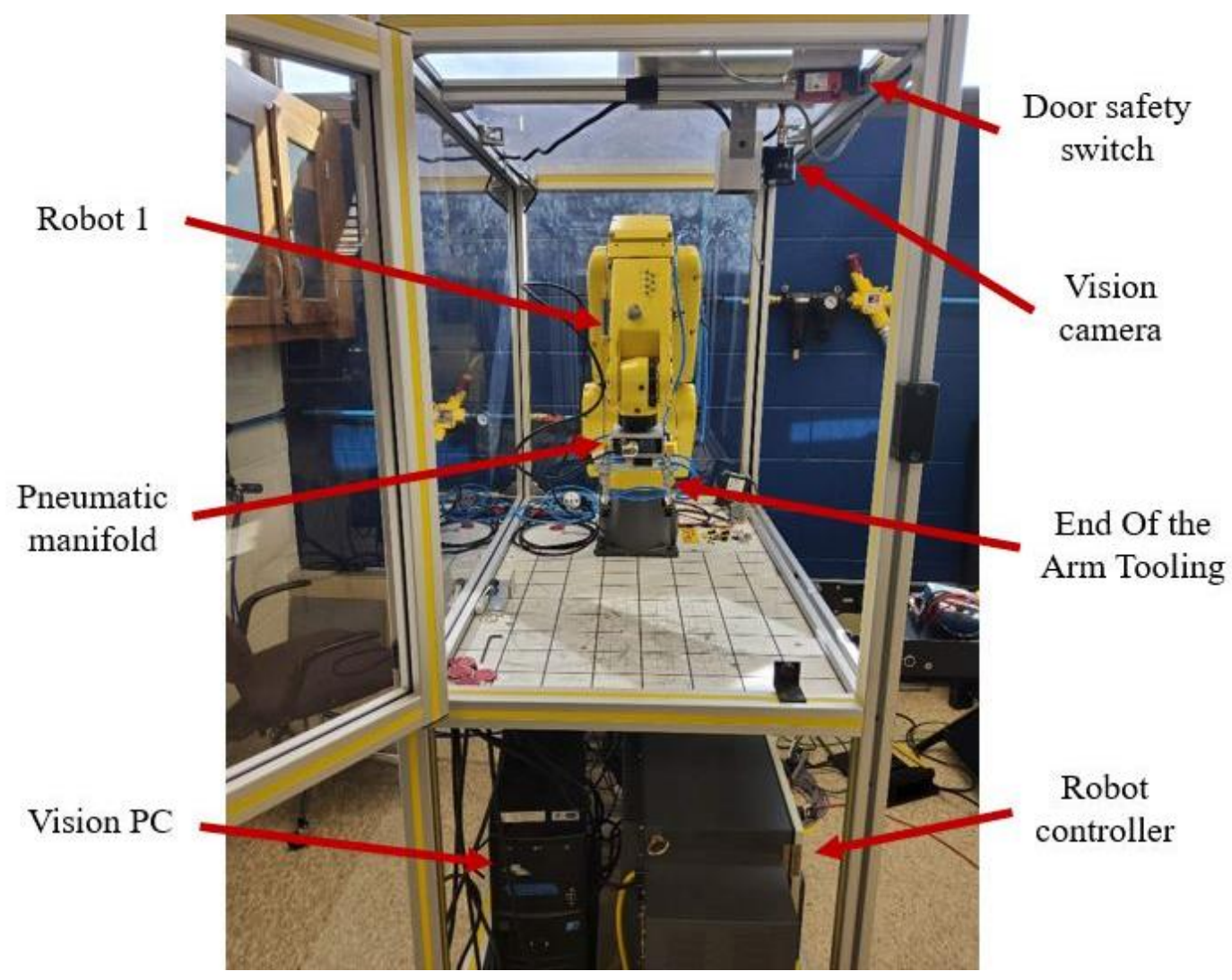

Figure 5 Quality check station

\subsubsection{Robot 1}

Robot 1 is the first robotic cell in the system. If the workpiece matches the specified dimensions during the quality check, the robot places it on the conveyor. The station has a six-axis FANUC LRMate200iC robot with a five $\mathrm{kg}$ payload mounted on a FANUC training cart, as shown in Figure 5. The robot is enclosed and equipped with a door safety switch for additional protection.

Robot 1 uses an R30iA Mate controller with handling tool package V7.30 and R538 EthernetIP adapter software to enable communications with the master PLC. The robot has a teach pendant for online programming and also supports FANUC Roboguide simulation software for offline programming.

\subsubsection{Robot 1 end of the arm tooling}

The robot uses vacuum suction end of the arm tooling, as shown in Figure 6, to grab the workpiece. The gripper has suction cups mounted on spring-loaded buffers, keeping the cups in place by pushing them down on the workpiece. The buffers are attached to an extruded aluminum frame by brackets. 


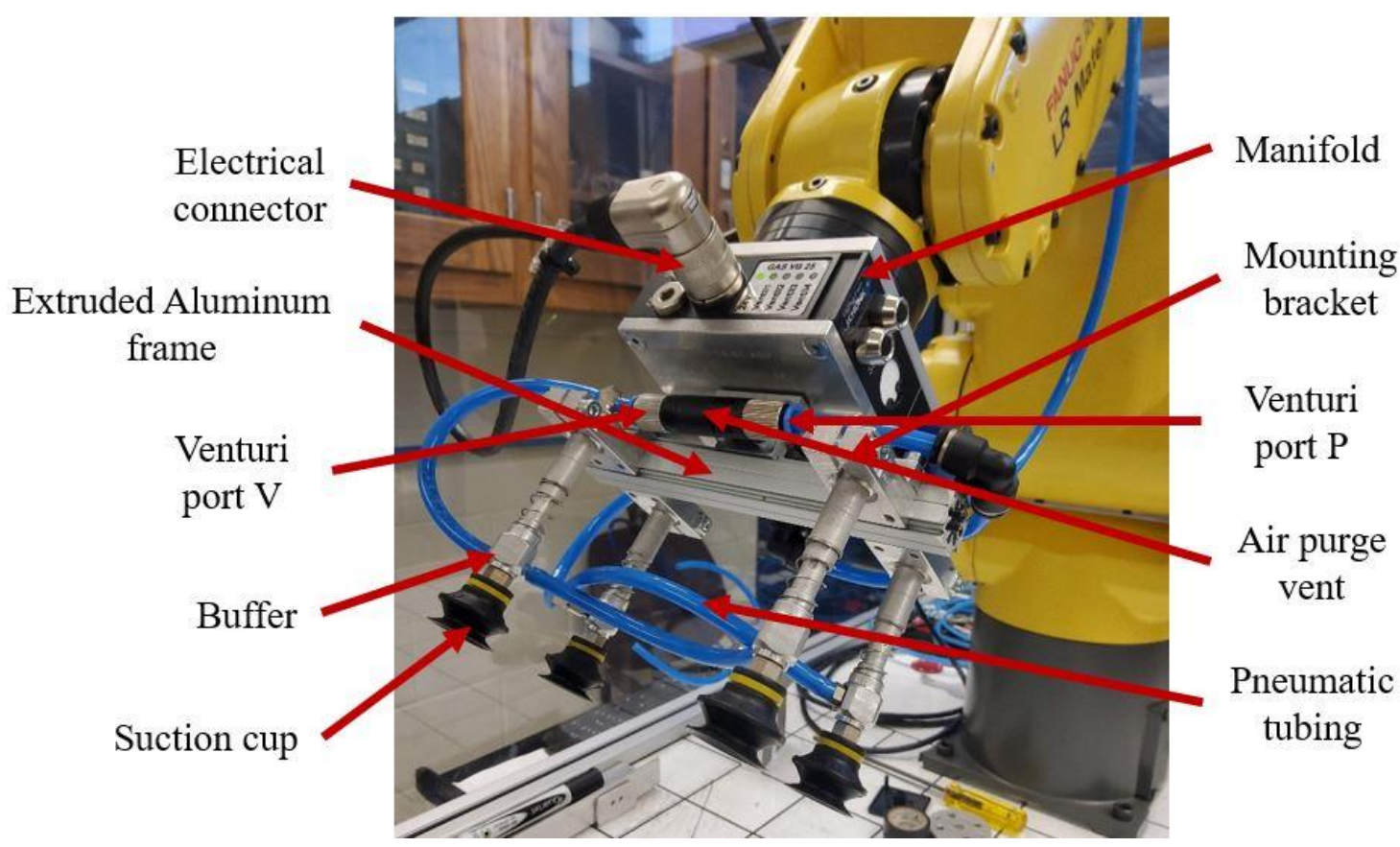

Figure 6 Robot 1 End Of the Arm Tooling

The end effector uses two channels from a Schunk Ventiblock VB25 manifold to control positive pressure to a venturi tube port $P$. The venturi purges air and generates a vacuum on port $\mathrm{V}$. When the pair of suction cups are connected to port $\mathrm{V}$ by pneumatic tubing engages with the workpiece, the vacuum generated by the venturi grips the workpiece. The robot now picks the workpiece held firmly by the suction cups.

\subsubsection{Vision system}

The vision system uses a SONY XC-56 black and white CCD camera with a 659 X 494pixel array. The spotlight is a high-intensity white LED light used to illuminate the object uniformly. The camera and spotlight are both mounted on the top of the cart, as shown in Figure 4.

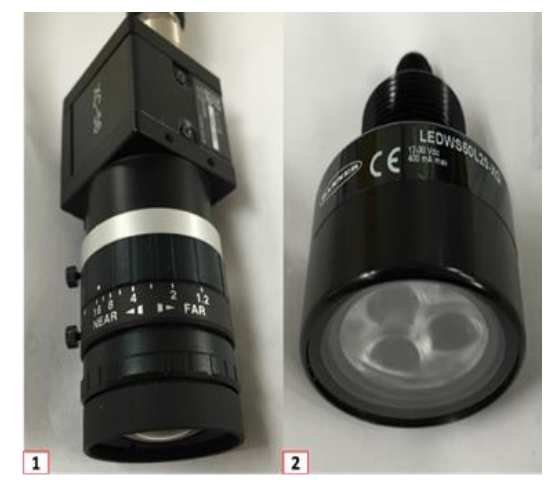

Figure 7 [1] SONY XC-56 camera [2] LED spotlight 
The robots have FANUC 2D iRVision system as the processing software. The Vision PC mounted on the cart is connected to the robot controller via an ethernet cable enabling the communication

\subsection{Material handling subsystem}

The material handling subsystem, shown in Figure 8, is responsible for transferring the workpiece between various stations and consists of a belt conveyor, a servo conveyor, and Donald Engineering Station 1.

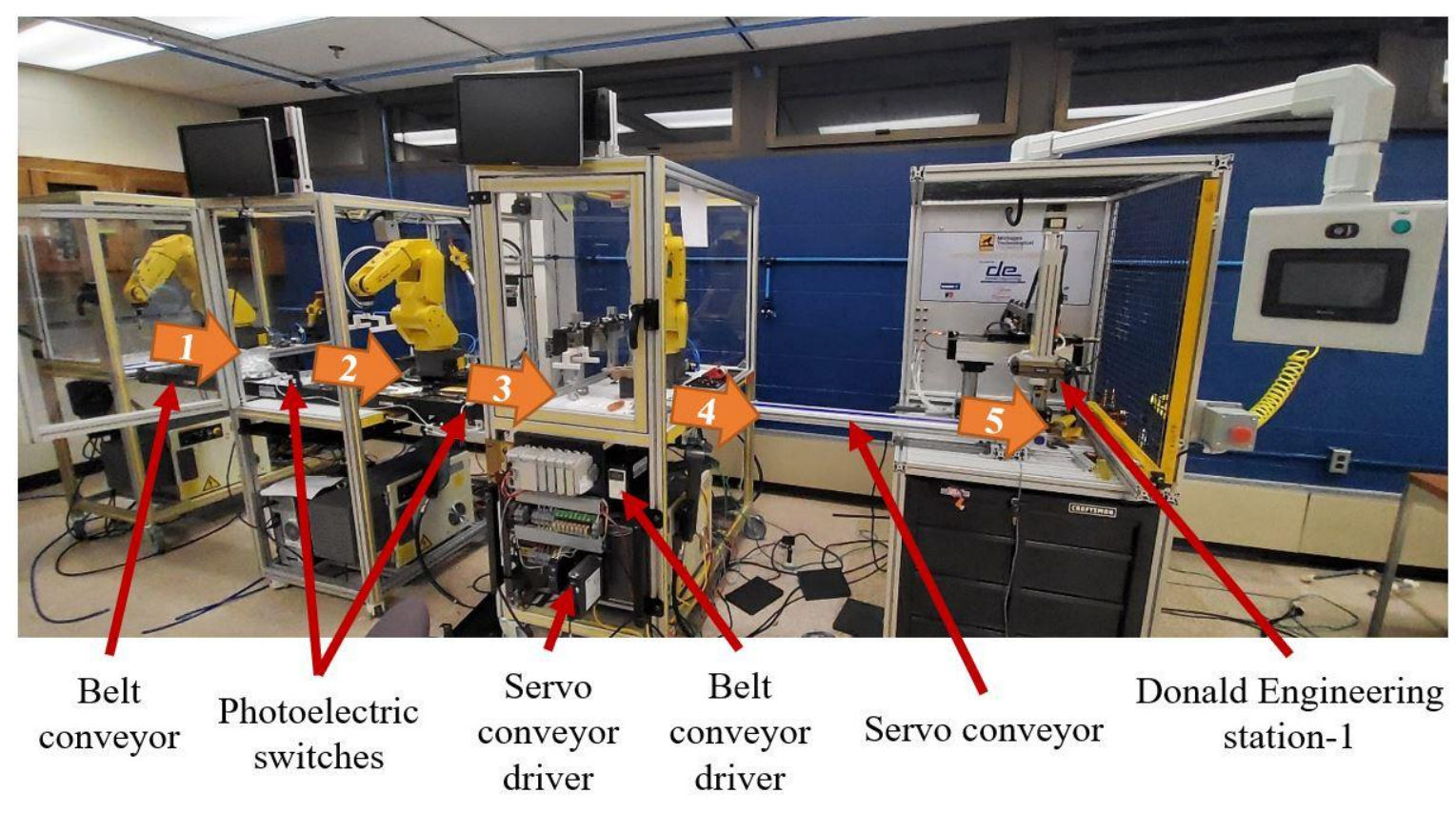

Figure 8 Material handling subsystem operation

The sequence of operation of this subsystem is as follows:

1. Robot 1 places the workpiece on the place fixture kept on the belt conveyor at position one upon the workpiece passing the quality check.

2. The belt conveyor moves the workpiece until being detected by the photoelectric sensor at position two, and Robot 2 picks up the workpiece for machining.

3. After the workpiece is machined, Robot 2 places it back on the belt conveyor.

4. The belt conveyor moves the workpiece until being detected by the inductive proximity sensor at position three. It then stops, and robot-3 picks up the workpiece.

5. Robot 3 picks up the workpiece from position three and places it on the servo conveyor at position four. 
6. The servo conveyor moves the workpiece from position four to position five, where Donald Engineering station one picks it up and places the workpiece to be picked by the operator.

7. The belt conveyor moves from Robot 3 to Robot 1 until the inductive proximity sensor detects the place fixture at position one.

\subsubsection{Belt conveyor}

The belt conveyor transfers the workpiece between the quality check and machining subsystems (Robots 1,2, and 3). The belt conveyor is a Mini mover conveyor LITE series model and comes with a geared brushless DC motor to move the belt and a variable speed drive to control direction and speed.
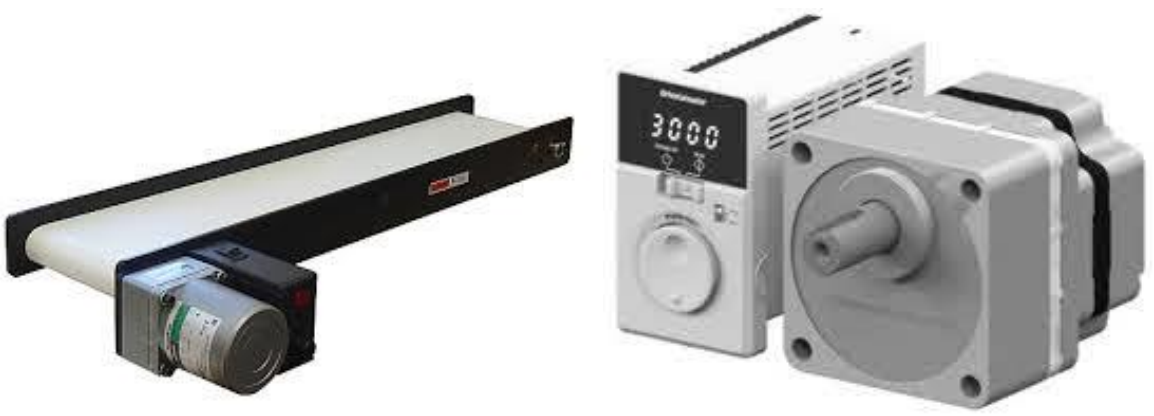

Figure 9 Belt conveyor, driver, and motor

After Robot 3 picks the workpiece, the conveyor reverses direction and moves from position three to position one, as shown in Figure 8. The master PLC controls the conveyor by using on/off and forward/reverse commands.

\subsubsection{Inductive proximity sensors}

The inductive proximity sensors detect the place fixture and signal the master PLC to stop the belt conveyor. The sensor is Kissling L408 inductive proximity sensor and works by generating a high-frequency magnetic field and sensing the disturbances.

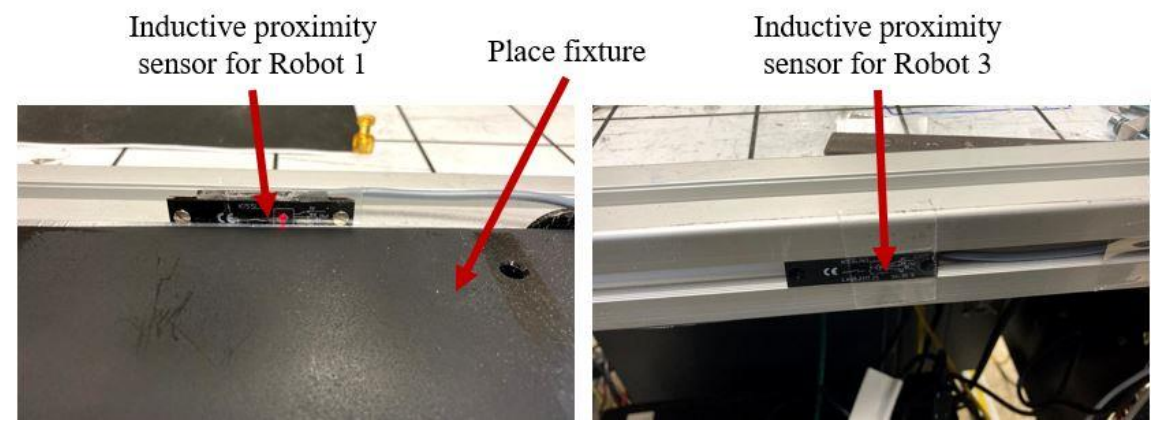

Figure 10 Inductive proximity sensors 
The sensors are wired to the master PLC on the digital input card and are mounted near Robot 1 and Robot 3 at positions one and three, shown in Figure 8.

\subsubsection{Place fixture}

The place fixture kept on the belt conveyor transports the workpiece to various robotic stations. The workpiece is $20 \mathrm{~mm}$ thick, which is lower than the photoelectric sensor's minimum detection height, resulting in the workpiece not being detected. The robots in the process place the workpiece on the place fixture to account for the low height resulting in detection by the photoelectric sensors.

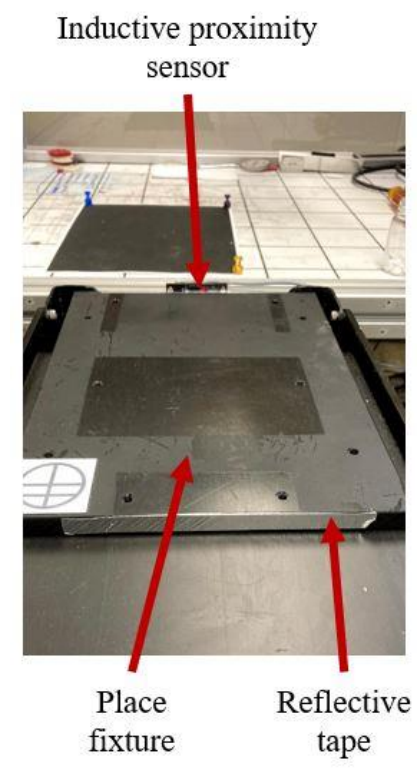

Figure 11 Place fixture

The place fixture has reflective tape on it, which enables detection by the inductive proximity switches.

\subsubsection{Photoelectric sensor}

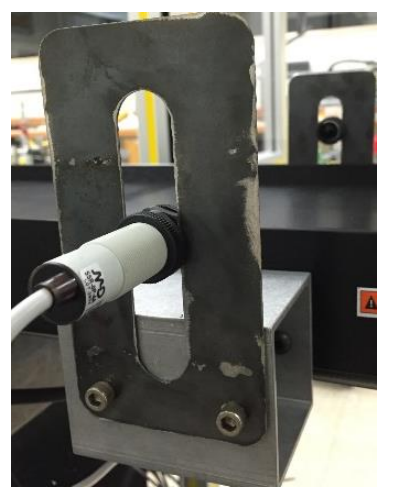

Figure 12 Photoelectric emitter and receiver 
The photoelectric sensors detect objects on the belt conveyor. They are mounted near Robot 2 and Robot 3 on the height-adjustable brackets to account for various workpiece heights.

The photoelectric sensors come in a pair of an emitter and a receiver. The emitter sends infrared light to the receiver placed opposite it. When the workpiece traveling on the belt conveyor cuts the beam of infrared light, a $24 \mathrm{~V}$ digital output is sent to the master PLC stopping the belt conveyor.

\subsubsection{Servo conveyor}

The servo conveyor is a Schunk Delta series linear actuator, controlled by an Applied Motion SV2A3 IP-DE servo driver mounted between Robot 3 and Donald Engineering Station one. It comes with a roller guide-based guidance system and a toothed belt drive connected to a servo motor. The servo driver accepts commands from the master PLC over EthernetIP.
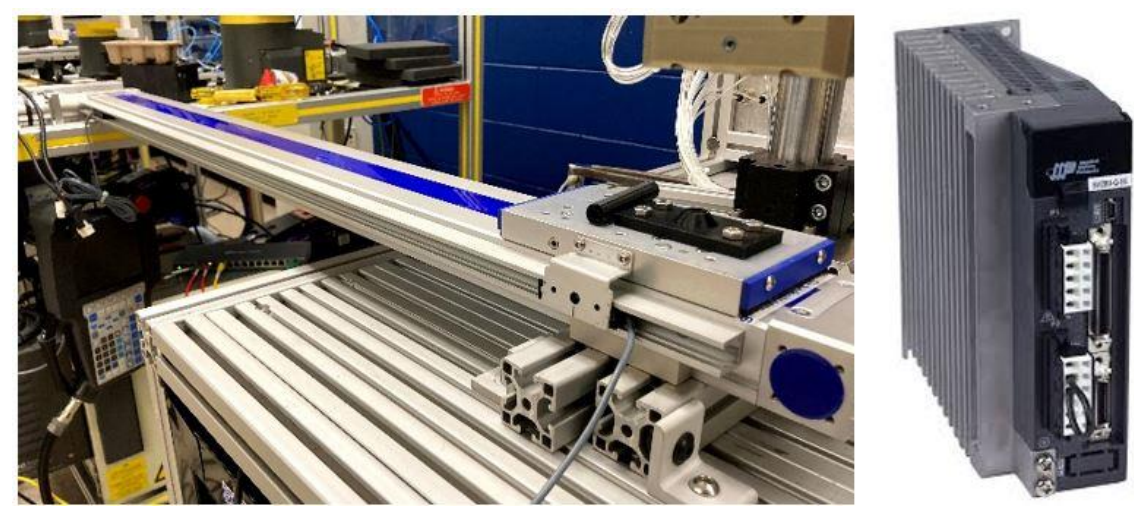

Figure 13 Servo conveyor and servo driver

The servo conveyor transports the workpiece from Robot 3 to Donald Engineering station one. When Robot 3 places the workpiece on the servo conveyor, it receives a command from the master PLC to move from position four to position five, as shown in Figure 8. The rotary encoder on the motor shaft sends the conveyor position feedback to the master PLC and stops the conveyor at desired positions.

\subsubsection{Donald Engineering station one}

The Donald Engineering station one, abbreviated as DE station-1, is a four-axis prismatic robot. The robot is assembled and fabricated by Donald Engineering from Grand Haven, Michigan. The X, Y, R axis at the wrist are Schunk pneumatic actuators, and Z is a Schunk electric actuator shown in Figure 14. The robot is mounted on a station fabricated using $\mathrm{T}$ slot extruded aluminum parts.

The station has multiple safety features which allow for a safe operation. The station has a light curtain in front that will cut the air supply to the pneumatic actuators when crossed. A mushroom-style emergency push button on the front of the station, when pressed, will 
purge all the air from the system and disable the robot. All sides are enclosed with a safety fence to prevent entry into the operating area.

The operator controls the robot actuators with a boom-mounted Unitronics HMI and PLC. The Unitronics PLC supports two-way communication with the master PLC over EthernetIP communication protocol. This feature enables the robot actuators to be controlled remotely by receiving commands from the master PLC over EthernetIP.

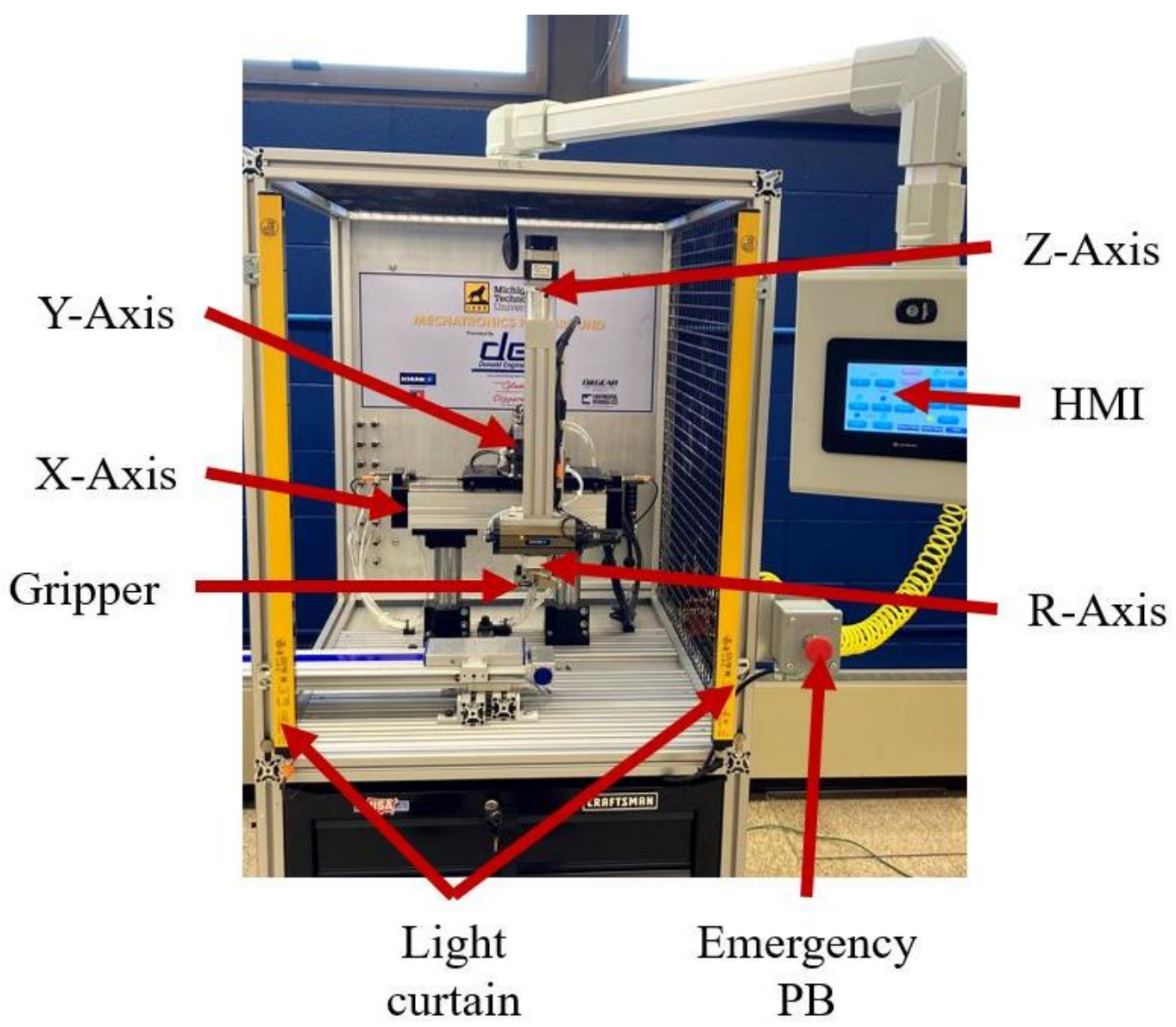

Figure 14 Donald Engineering station-1

The Donald Engineering station is the final part of the process. The workpiece is picked by the DE station from position five, shown in Figure 8, and delivers to the operator.

\subsection{Machining subsystem}

The purpose of the machining subsystem, comprised of Robot 2 and Robot 3, is to machine the workpiece according to the given specification. The end of the arm tooling on Robot 3 has a quick tool changer attachment to enable rapid tool swap to accommodate various material handling functions. 


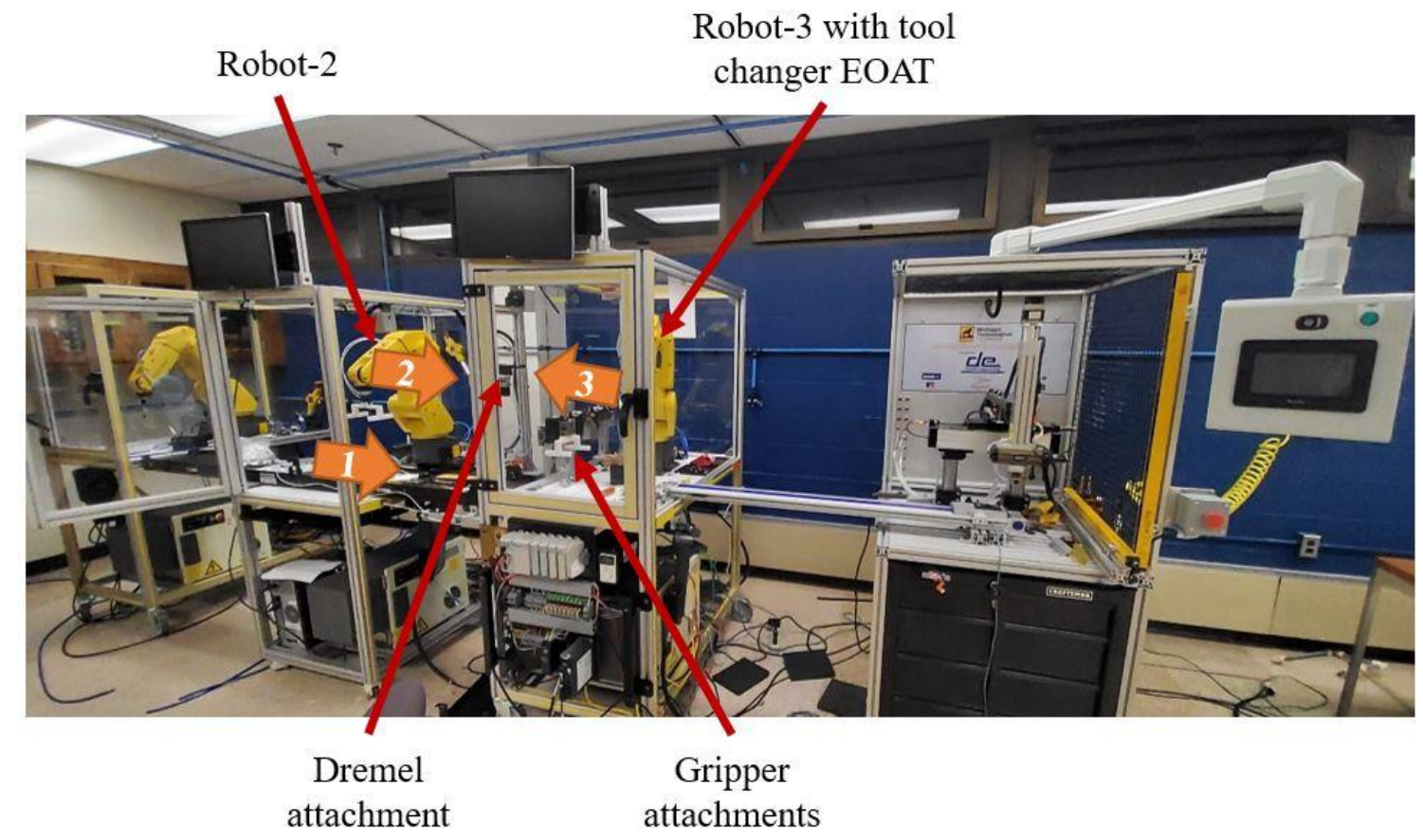

Figure 15 Machining subsystem layout

The subsystem follows the sequence of operation shown below,

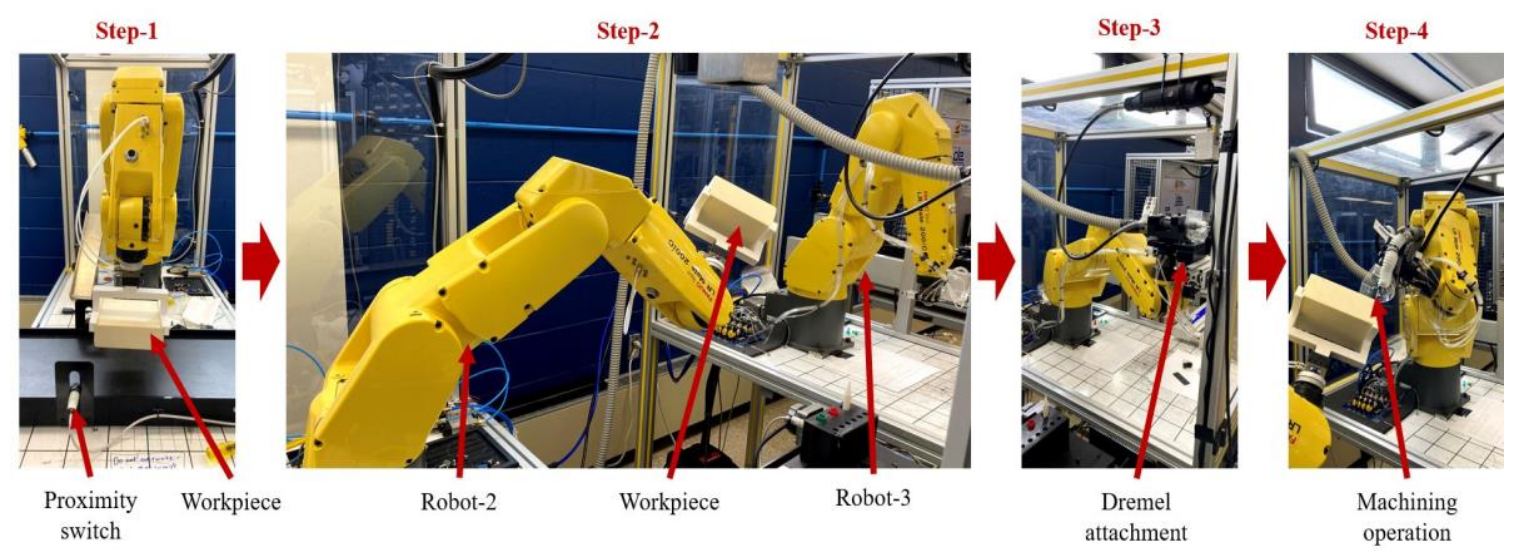

Figure 16 Sequence of machining operation

1. The workpiece passes in front of the proximity sensor, which transmits a 24VDC signal to the master PLC. The master PLC stops the belt conveyor, and Robot 2 picks the workpiece off the belt conveyor from position one, as shown in Figure 15

2. Robot 2 holds the part at position two to be machined by Robot 3, shown in Figure 16 
3. Robot 3 uses Dremel attachment to machine predefined by the user pattern

4. Once operation in step 3 is complete, the Robot 3 places the Dremel attachment back in its storage rack

\subsubsection{Robot 2}

Robot 2 has similar to Robot 1 specifications and hardware and software options installed.

\subsubsection{Robot-2 End Of the Arm Tooling}

Robot 2 is equipped with a gripper end of the arm tooling for picking and placing the workpiece on the conveyor, as shown in Figure 17. The gripper is a Schunk PGN-40 pneumatic gripper operating on $80 \mathrm{psi}$. The pneumatic lines provide the air supply to the robot.

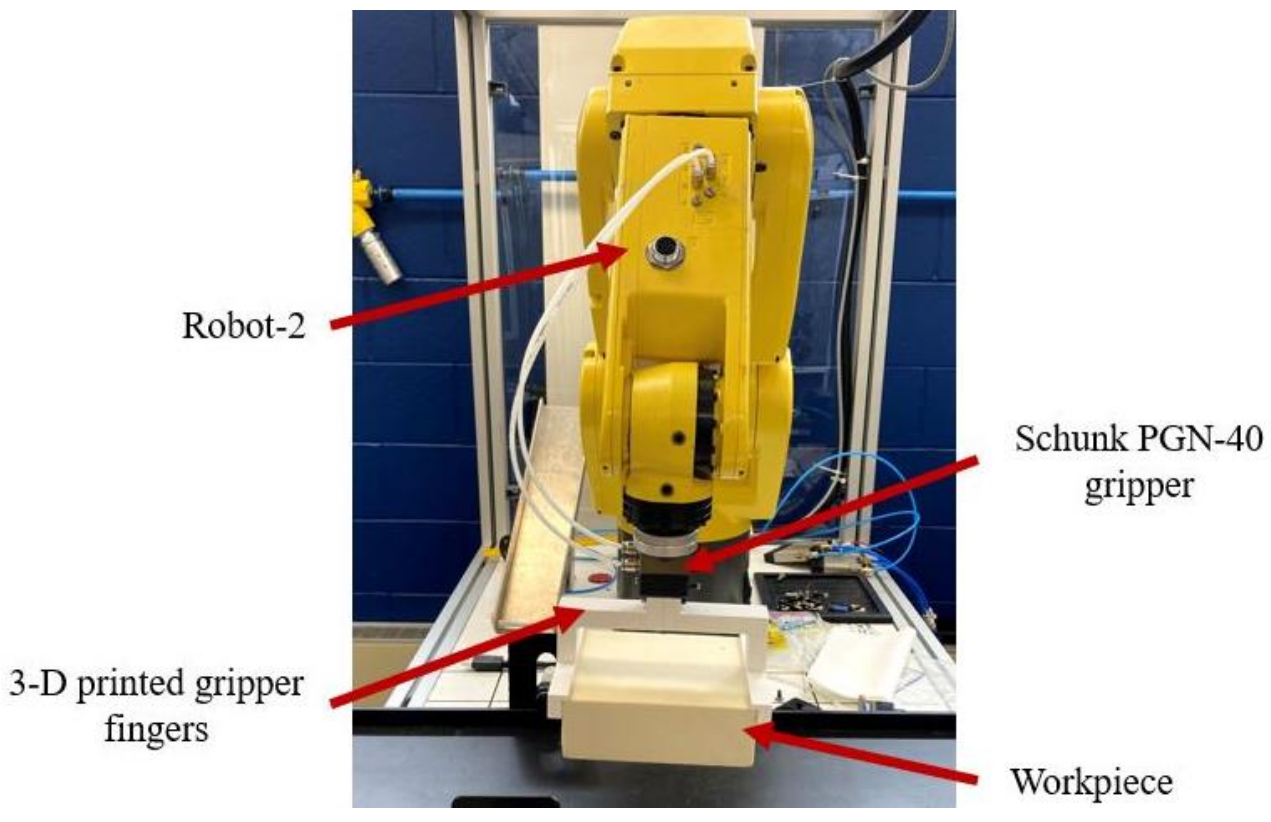

Figure 17 Robot-2 End Of the Arm Tooling

The gripper has custom 3-D printed fingers to adjust for the size of the workpiece. When closed, the fingers can hold a workpiece up to $150 \mathrm{~mm}$ in length.

\subsubsection{Robot 3}

Robot 3 is the third robotic cell in the system and has similar hardware and software specifications to Robot 1 and 2. The robot performs machining operations on the workpiece. After the machining is complete, the robot picks up the machined workpiece from the belt conveyor and places it on the servo conveyor. 


\subsubsection{Robot-3 End Of the Arm Tooling}

The Robot 3, equipped with a quick tool changer, machines the workpiece using the Dremel tool and transfers the workpiece on the servo conveyor.

The end of arm tooling on Robot 3 is a Schunk quick-change ZK master, shown in Figure 18. The ZK master allows the robot to lock to various tool changer attachments. The locking and unlocking action on the quick-change master is pneumatically controlled.

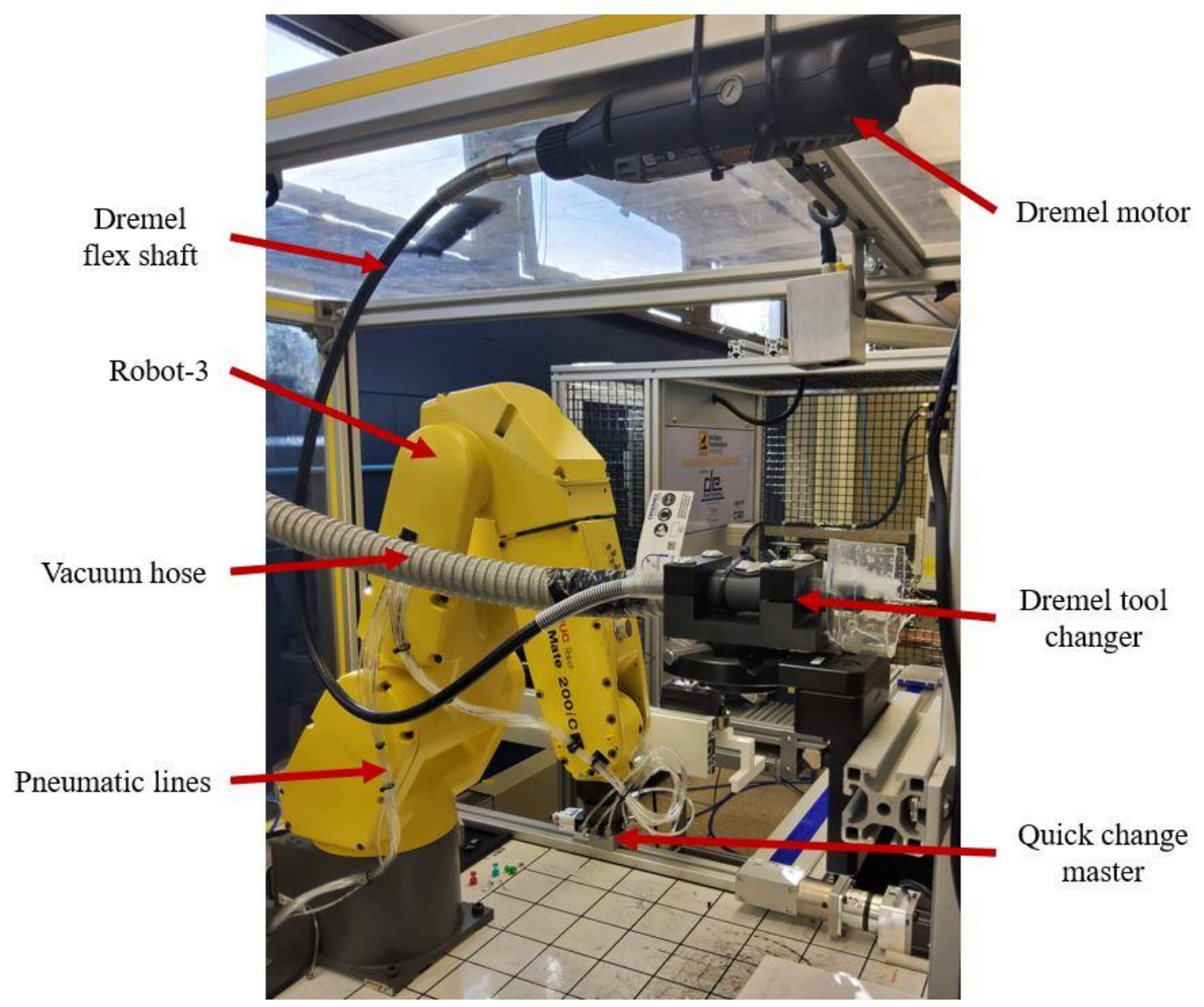

Figure 18 Robot-3 workcell

A Clippard EMC-08-24 electronic manifold controls the air supply to the quick change master, as shown in [1] from Figure 19. The manifold has pre-assembled eight valves on a $\mathrm{PCB}$ with reverse polarity protection. 


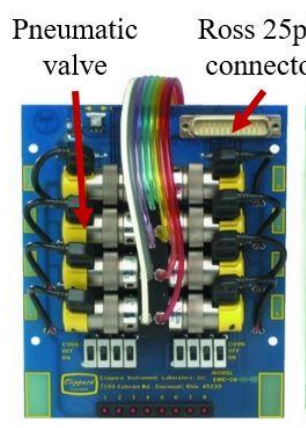

1

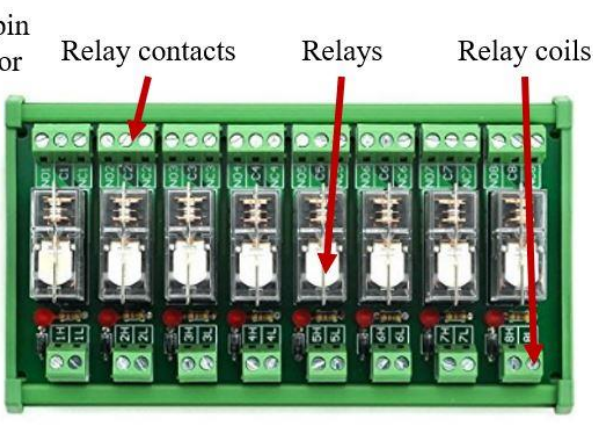

2

Figure 19 [1] Electronic manifold [2] Relays

These individual valves control is through a Ross 25-pin connector that accepts -24VDC as signal and $+24 \mathrm{VDC}$ as common. The $-24 \mathrm{VDC}$ signals are passed through the relay contacts as shown in [2] from Figure 19. The relay coils are wired to Robot 3 digital outputs on the robot controller.

\subsubsection{Dremel Tooling}

Robot-3 uses the Dremel tooling for machining the workpiece. Figure 20 shows the variable speed Dremel tooling system equipped with the flex shaft with the spindle.

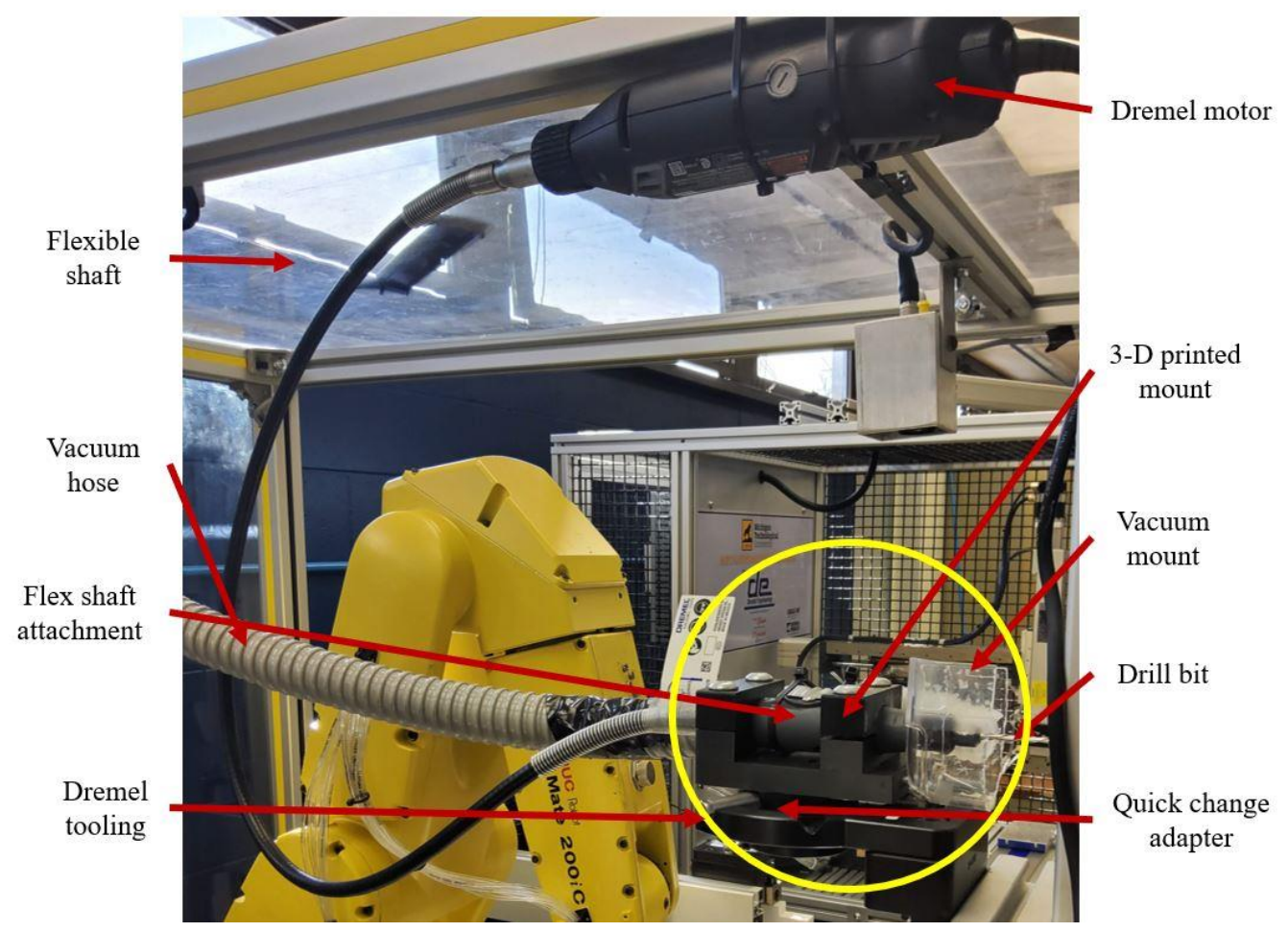

Figure 20 Dremel tooling 
The Dremel motor is switched ON/OFF by Robot 3 via a relay. The flex shaft attachment houses a 1/8 inch two-flute drill bit on its chuck with a mount for a vacuum hose. The flex shaft attachment is clamped to a 3-D printed mount bolted to a Schunk quick-change ZA adapter.

\subsubsection{Gripper tooling}

Robot 3 uses a Schunk PGN64 pneumatically actuated gripper to pick the belt conveyor's workpiece and place it on the servo conveyor. When the robot attaches the gripper tooling to the ZK master, the air for open and close gripper functions is passed via the ZK master to the ZA adapter via air supply channels.

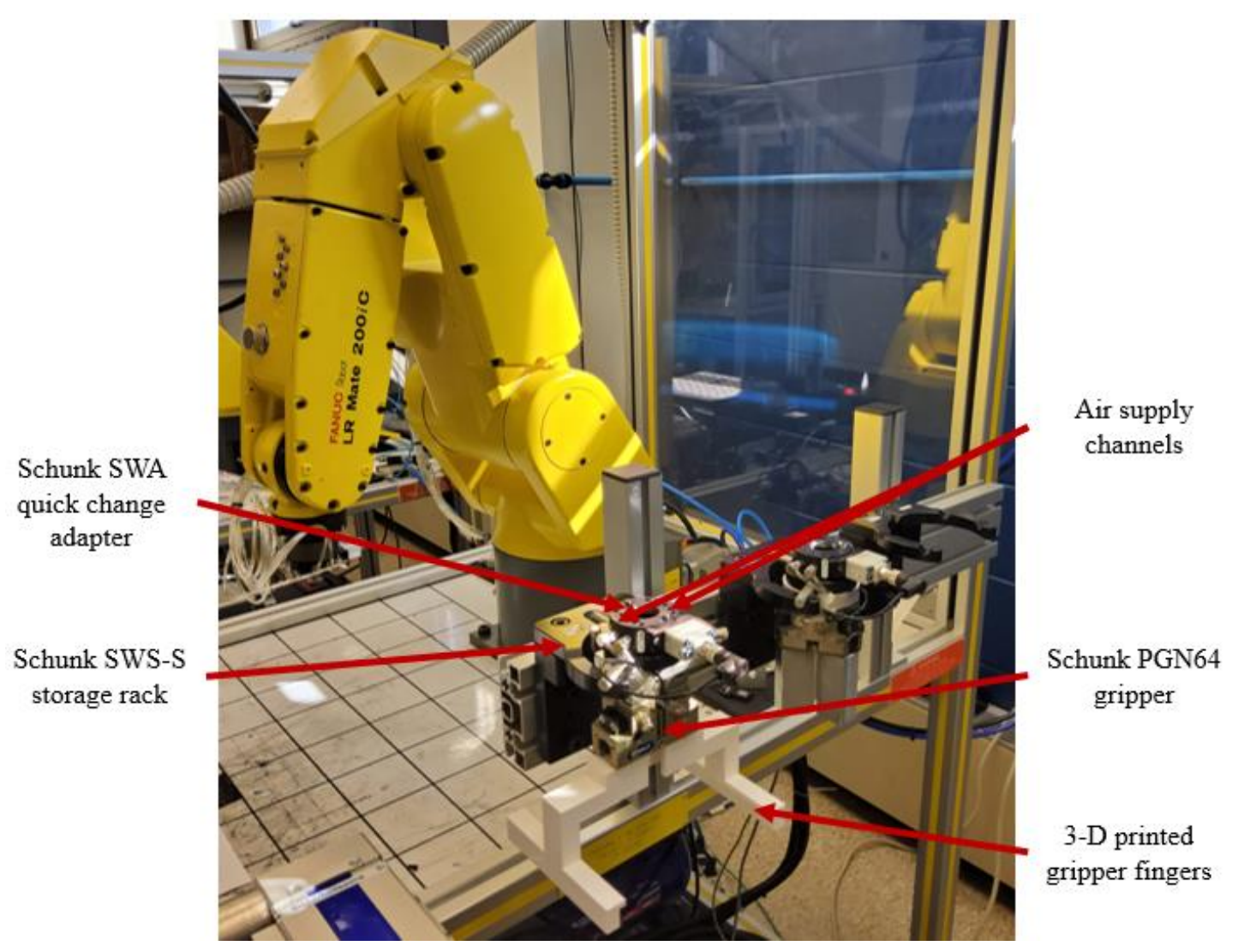

Figure 21 Gripper tooling 


\subsubsection{Vacuum}

The dust generated during the machining process needs to be evacuated from the workcell to keep it clean. A special-purpose vacuum cleaner with the extension hose secured on the Dremel tooling performs this function

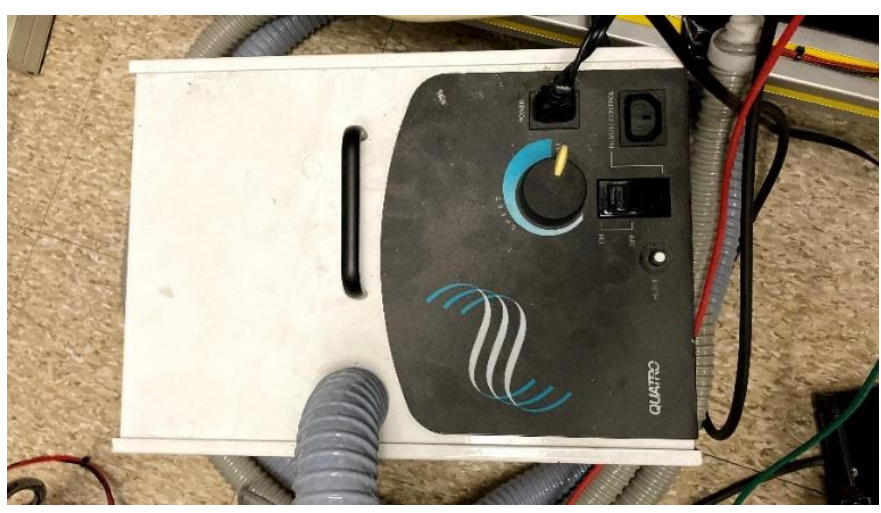

Figure 22 Vacuum

The vacuum is controlled by routing $110 \mathrm{~V}$ single phase through relay contacts and controlling the coil by the master PLC.

\subsection{Control and communication subsystem}

The process involves all the subsystems working together to perform quality control, material handling, and machining requires interconnectivity and coordination. The control and communication subsystem is responsible for providing a central control through the master PLC.

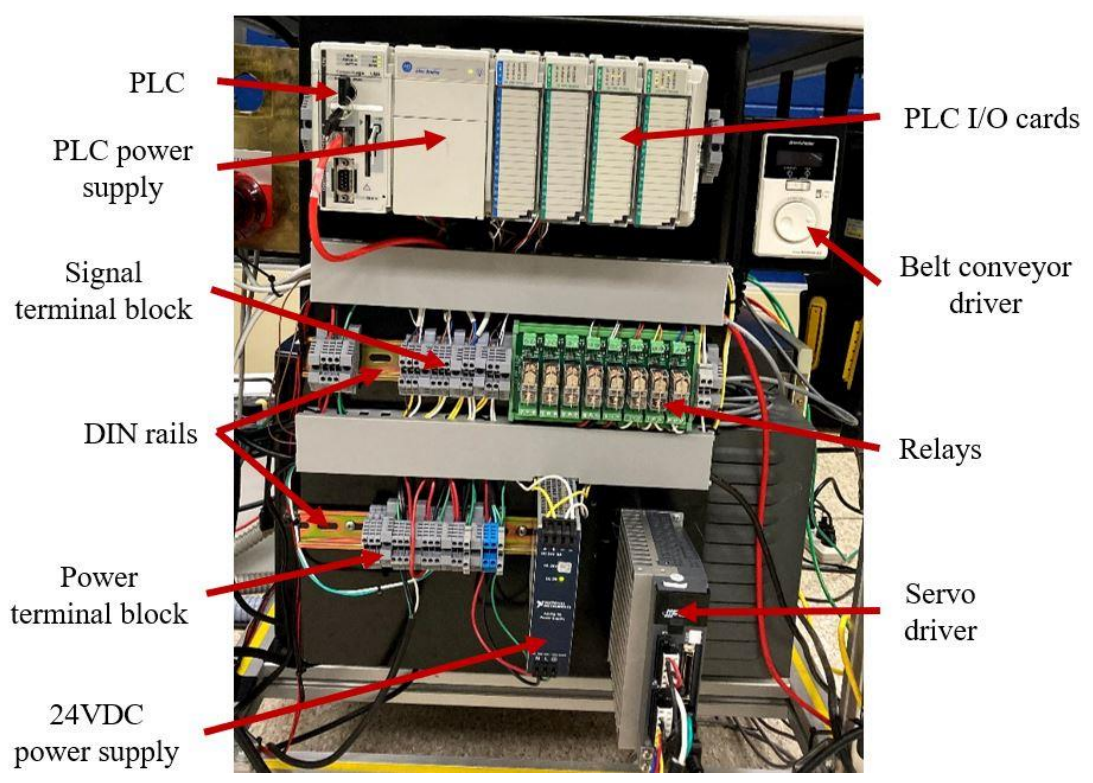

Figure 23 Control and communication subsystem 
An electric panel houses all the hardware for controlling the process, as shown in Figure 23. The panel has terminal blocks that transfer feedback from the photoelectric sensors and inductive proximity sensors to the master PLC I/O card. The relays control the Dremel tooling, vacuum, and the quick tool changers on Robot 3.

The servo drivers and belt conveyor drivers that control the conveyors from the material handling subsystem are mounted on the panel, as shown in Figure 23. The 24VDC power supply powers components running on 24VDC and transfers 24VDC control signals through the relay contacts.

\subsubsection{Master PLC}

The control subsystem has a Rockwell Automation Allen Bradley L32E PLC functioning as the master controller. The PLC schedules the execution and selection of programs on all subsystems. The PLC supports communication over EthernetIP, a communication protocol by Open DeviceNet Vendors Association (ODVA) and supported by Rockwell Automation.

Robots 1, 2, 3, and the servo driver are capable of two-way communication over EtherNetIP. The Donald Engineering station one is controlled by a Unitronics PLC, which also supports EtherNetIP. The master PLC communicates with these stations over EtherNetIP. The belt conveyor and the photoelectric sensors are connected to the PLC I/O cards using hardwired connections.

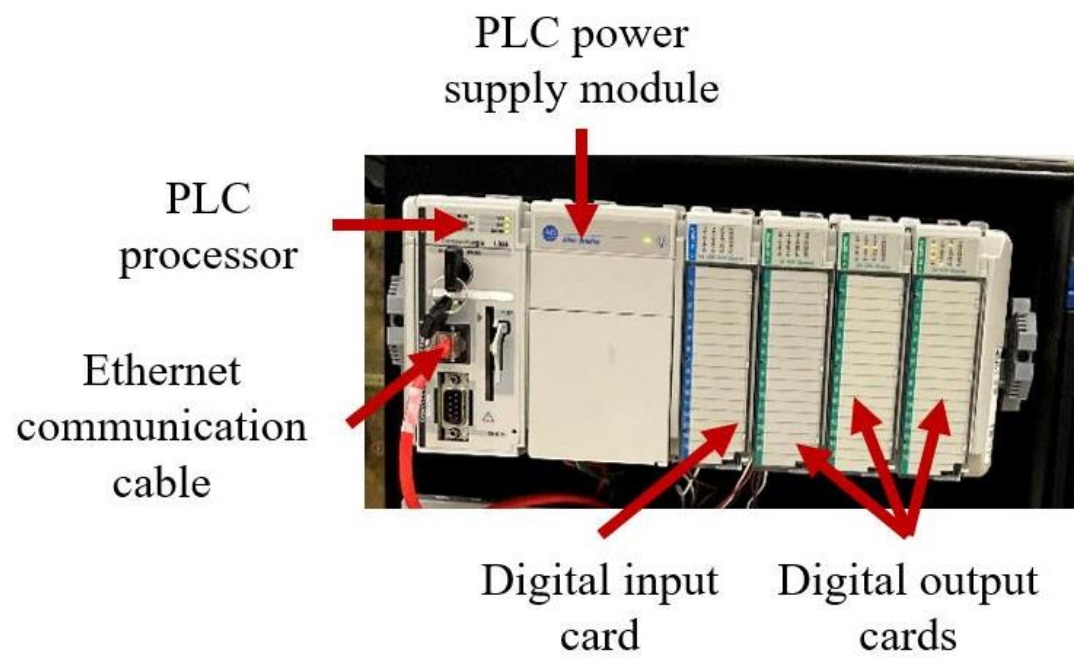

Figure 24 Master PLC schematics

The PLC processor has a power supply module running $120 \mathrm{~V}$. It also has one digital input card, two digital output cards mounted on the chassis. The PLC runs firmware V20.00 and is programmed using Rockwell RSLogix 5000 software. 


\subsubsection{Terminal blocks}

Terminal blocks mounted on DIN rails act as peripheral to connect the sensors and devices to the master PLC I/O cards. The feedbacks from photoelectric sensors and inductive proximity sensors are transferred to the master PLC via the terminal block.

The signal terminal blocks connect the inputs from photoelectric sensors one and two to the digital input card. The power terminal block connects a single-phase $110 \mathrm{~V}$ power supply to the servo diver, the $24 \mathrm{VDC}$ power supply, and the relay contacts. The relay contacts power the vacuum and the Dremel tooling, as shown in Robot-3 End Of the Arm Tooling.

\subsubsection{VDC power supply}

The power supply module is a National Instruments 24VDC power supply. The input for the module is single phase $120 \mathrm{~V} \mathrm{AC}$ and outputs $24 \mathrm{VDC}$ on two output ports. The photoelectric sensor and the PLC I/O cards are powered through the 24VDC power supply.

\subsubsection{Ethernet switch}

The 16-port TP-Link model TL-SG116 ethernet switch connects The switch connects Robots 1,2,3, the master PLC, Donald engineering station-1, and the robot vision system PCs, as shown in Figure 25.

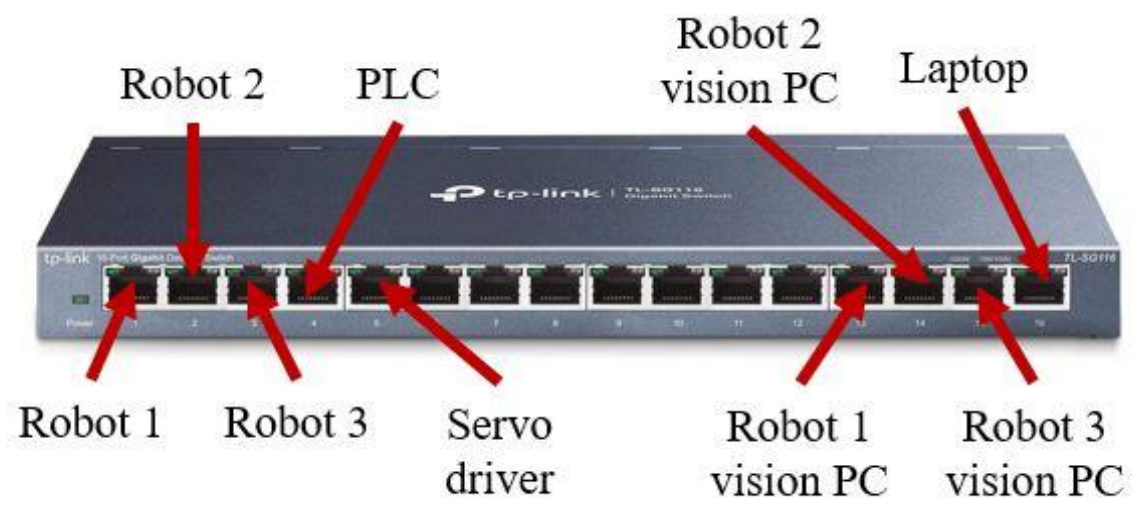

Figure 25 Ethernet switch 


\section{Communications layout}

The proposed project aims to integrate different subsystems and create a base for intersystem collaboration utilizing a communications network for information exchange. The communication network enables inter subsystem signal handshaking to obtain the device and program status for coordinating the process.

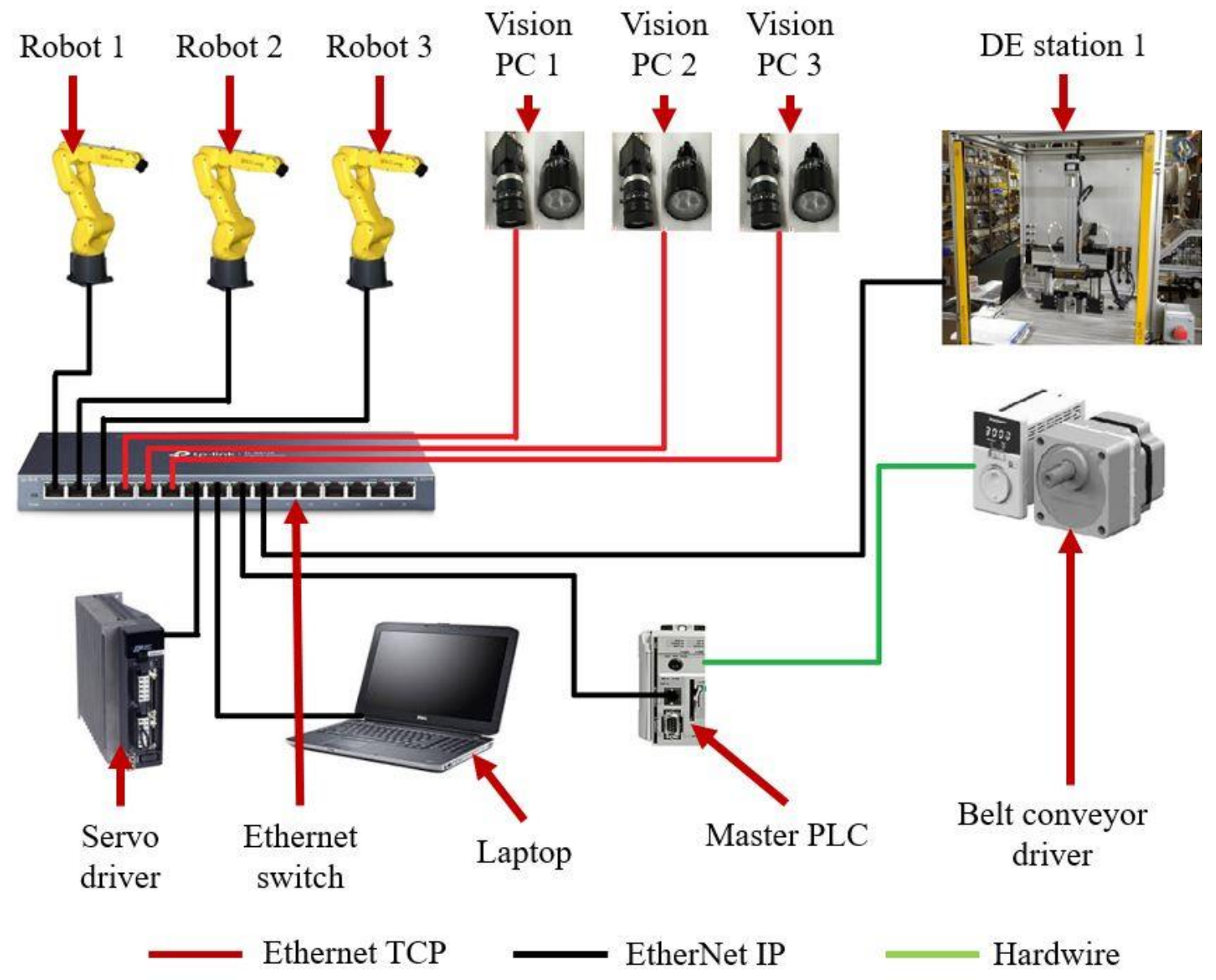

Figure 26 Communications layout

The majority of subsystems support Ethernet-based communication protocols such as a local area network (LAN) or a wide area network (WAN). All the devices shown in Figure 26 are connected using a twisted pair or a fiber optic cable for Ethernet communication.

All FANUC Robots, servo driver, the master PLC, DE station 1, and the laptop support communication over EthernetIP communication protocol. EthernetIP is a communication protocol developed by ODVA Inc. and is one of the most popular industrial protocols allowing the transport of I/O messages between devices. 
Ethernet TCP handles connections between the vision PC client and the robot controller server while the belt conveyor driver is connected to the PLC by hardwire connection to exchange 24VDC signals handshaking.

\subsection{Network topology and IP addresses}

Network topology is the arrangement of the elements in a communication network. It is essential to select the correct topology as it affects system performance and health. In the proposed system, all nodes are connected through an unmanaged Ethernet switch in a star topology which allows access to information from any node on the network. The star topology allows for simpler network architecture, accessible information access, fast data transfer speeds, and control from multiple locations. The IP addresses of each node on the network are shown in Table 1.

Table 1 System IP addresses

\begin{tabular}{lll}
\hline Sr No. & Component name & IP address \\
\hline 1 & Robot 1 & 192.168 .1 .21 \\
\hline 2 & Robot 2 & 192.168 .1 .22 \\
\hline 3 & Robot 3 & 192.168 .1 .23 \\
\hline 4 & Vision PC 1 & 192.168 .1 .24 \\
\hline 5 & Vision PC 2 & 192.168 .1 .25 \\
\hline 6 & Vision PC 3 & 192.168 .1 .26 \\
\hline 7 & DE Station 1 & 192.168 .1 .4 \\
\hline 9 & PLC & 192.168 .1 .11 \\
\hline
\end{tabular}

\subsection{Configuring EthernetIP on the Robots}

FANUC robots come equipped with Ethernet TCP support and do not support EthernetIP protocol by default. The additional EthernetIP adapter option (ID R538) is obtained from FANUC and installed on all robot controllers to enable communication between PLC and the robots. The following steps are executed on the teach pendant to install and enable the EthernetIP communication protocol

1. Insert the memory stick with the correct version of the core software

2. Go to $[\mathrm{MENU}]>[\mathrm{FILE}]>[\mathrm{S} / \mathrm{W}$ Install]

3. Press [F4 [CHOICE]] > [USB(UD1:)]

4. Press [F3 Done] 
5. Select the correct option from the left window

6. Enter the PAC code under the PAC window

7. Press [F4 AUTH]

8. Press [F2 INSTALL]

9. If asked, press [F5 AUTOUPDT]

10. Cold start the controller

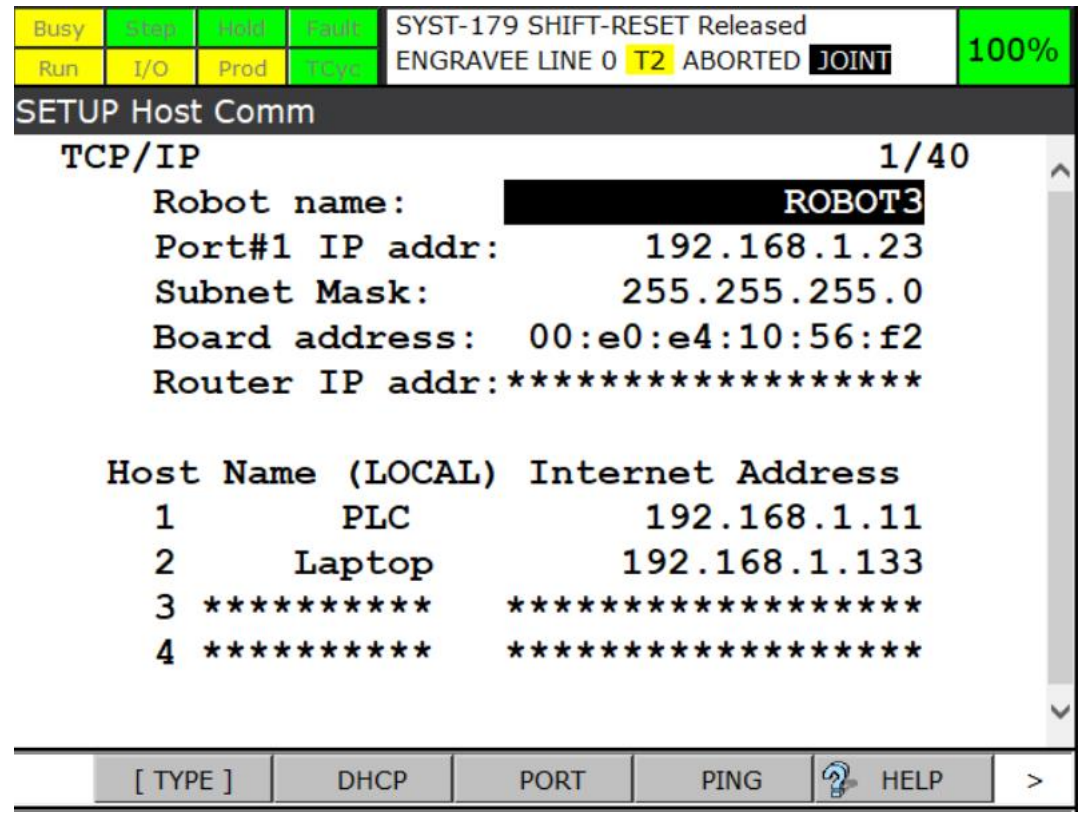

Figure 27 Host comms setup screen

After installing the EthernetIP adapter option on the controller, the robot is configured for communication over EthernetIP. Below are the steps to set the IP address through the teach pendant

1. Go to $[\mathrm{MENU}]>[$ SETUP $]>[$ Host Comm $]$

2. Select TCP/IP. A window will open up, as shown in Figure 27

3. Enter the robot name as Robot 1, Robot 2, and Robot 3 for the respective robots.

4. Enter the IP for the robots as specified in Table 1

5. Enter subnet mask as 255.255.255.0

6. Under the host name, enter the PLC IP address in the first entry

7. Enter the laptop IP address under the second entry

8. To confirm the availability of these devices on the network, press F4 to ping each IP

9. If the device is available, then it will show a successful ping on the screen 


\subsection{Configuring EthernetIP on the master PLC}

The master PLC supports EthernetIP communications by default through RSLinx Classic software, the communication driver for all Rockwell Automation PLCs. The PLC browses for any nodes on the Ethernet switch through RSLinx Classic and can access all the devices connected on EthernetIP from Figure 26. The settings for RSLinx Classic are accessed through the Laptop, which has an IP address shown in Table 1. To set the Laptop IP address, follow the steps below,

1. Under Windows setting open Ethernet

2. Click on Change adapter options from related settings to open the network connections window

3. Click on Ethernet on the new window > Select Internet Protocol Version 4(TCP/IPv4)

4. Enter the IP address as shown in Figure 28

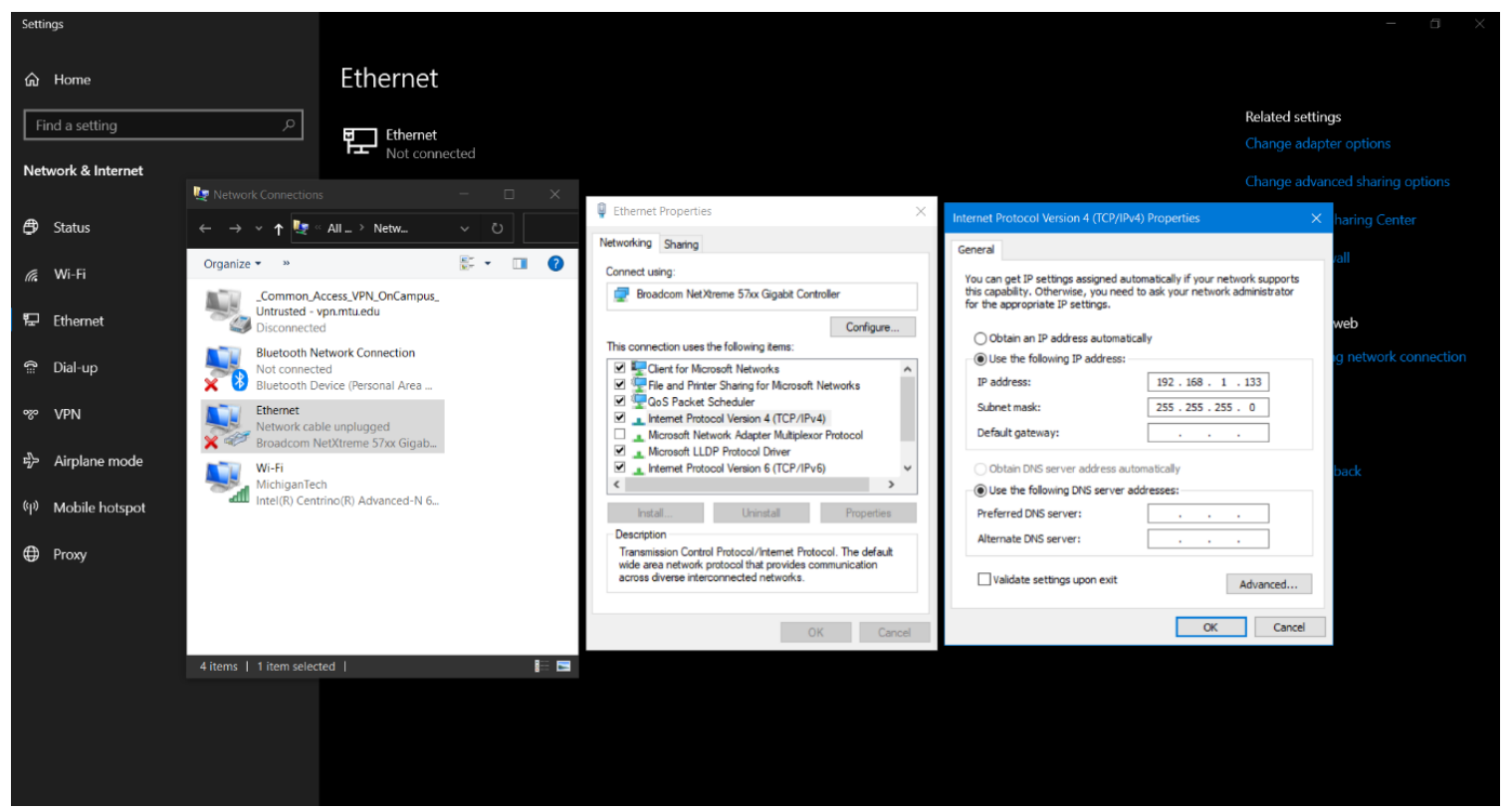

Figure 28 Setting the Laptop IP address

RSLinx Classic is configured after setting the Laptop IP address by following the steps below,

1. Open RSLinx Classic software from the start menu.

2. On the software, click on configure driver at position one, as shown in Figure 29, to open a new window

3. Select EtherNet/IP Driver from the Available Driver Types menu and click on Add at position two, as shown in Figure 29, to open a new window 


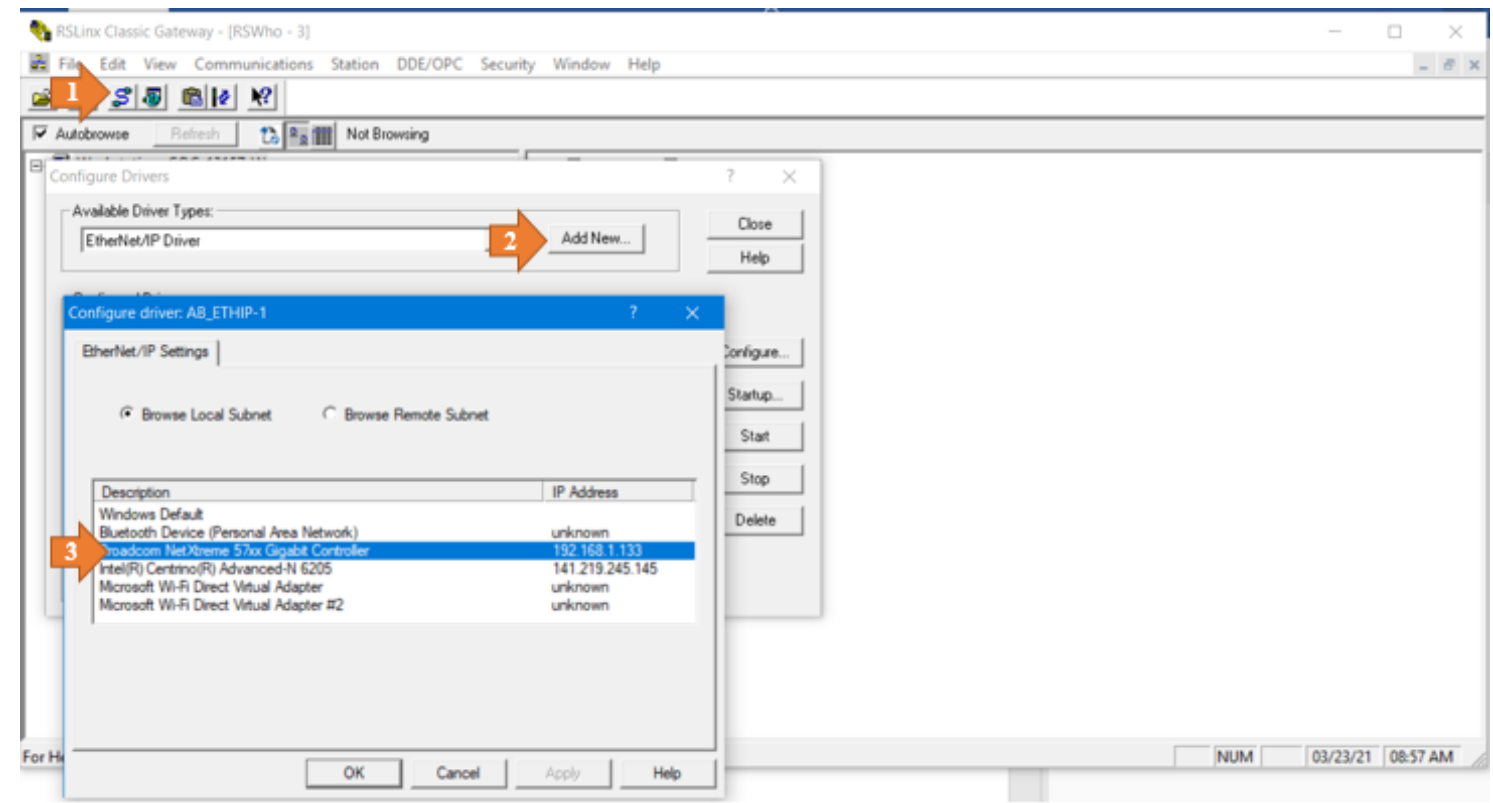

Figure 29 RSLinx Classic configuration

4. Select the Laptop IP address from the new window and click OK to finish

5. A correctly set EthetnetIP drive will show all the devices connected on EthernetIP from Error! Reference source not found. on the software as shown in Figure 30

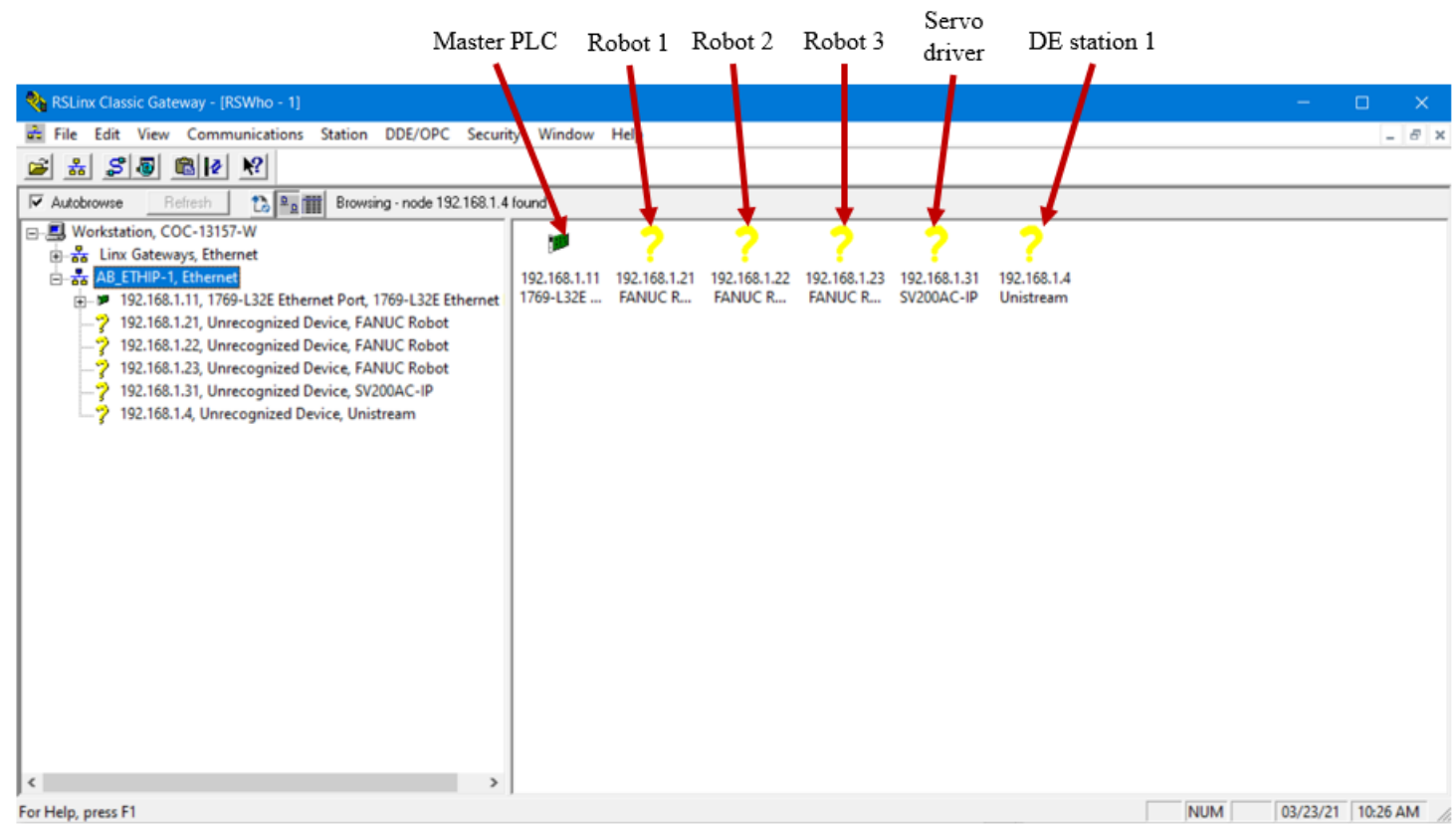

Figure 30 RSLinx classic network 


\subsection{Configuring EthernetIP on DE station 1}

A Unitronics USC-B5-B1 PLC controls the DE stations and supports EthernetIP communication by default.

The PLC's IP is set on the Unilogic programming software by going to Solutions Explorer $>$ PLC communications > Physical > Panel Ethernet > Properties Window, as shown in Figure 31. Under CPU Ethernet, put IP address as 192.168.1.4, Subnet mask as 255.255.255.0, Default Gateway as 192.168.1.1

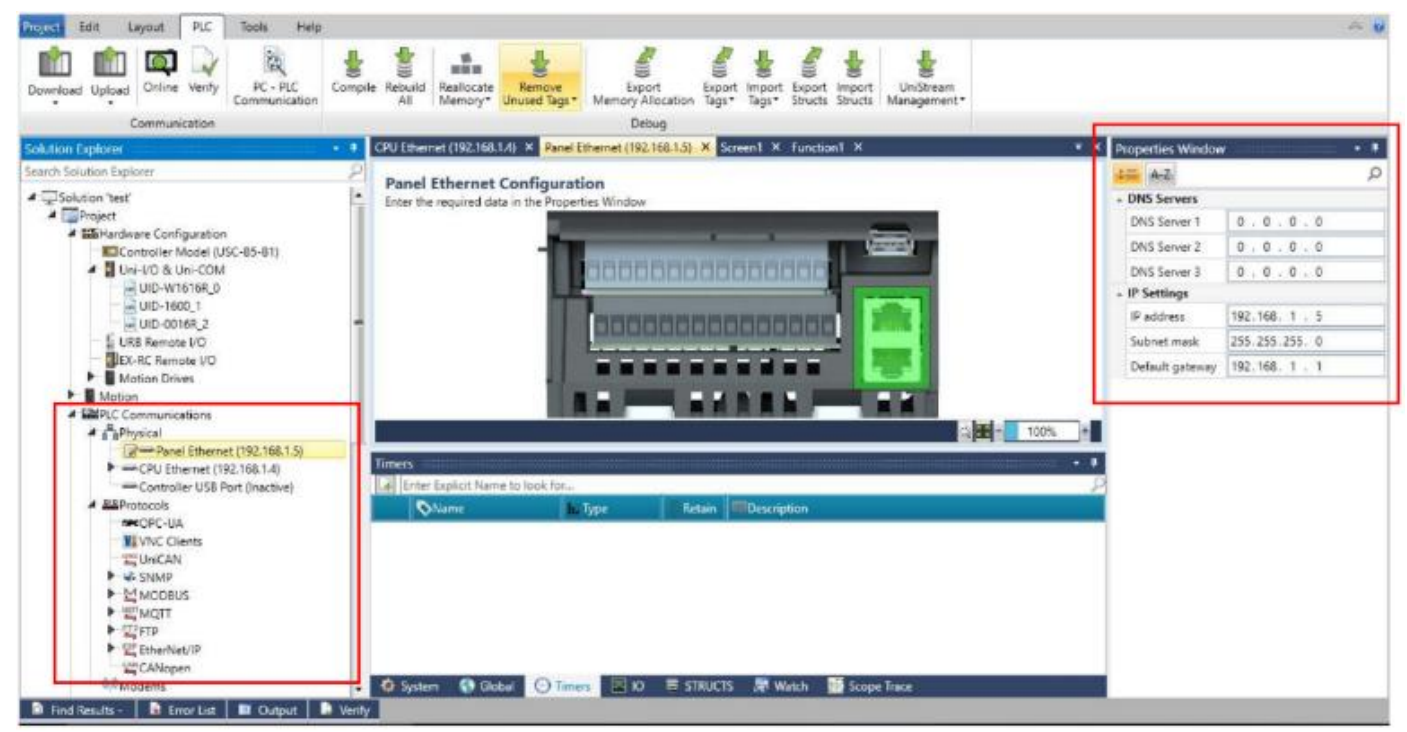

Figure 31 Unitronics PLC IP address configuration

The Unitronics hardware uses a VNC client-based communication. It has an IP address for a client to which both the HMI and the PLC can connect. To set the IP address for the VNC client go to Solutions explorer > PLC communications > VNC Clients > Connection 1 > Put IP Address as 192.168.1.6 > Tick show exit box as shown in Figure 32.

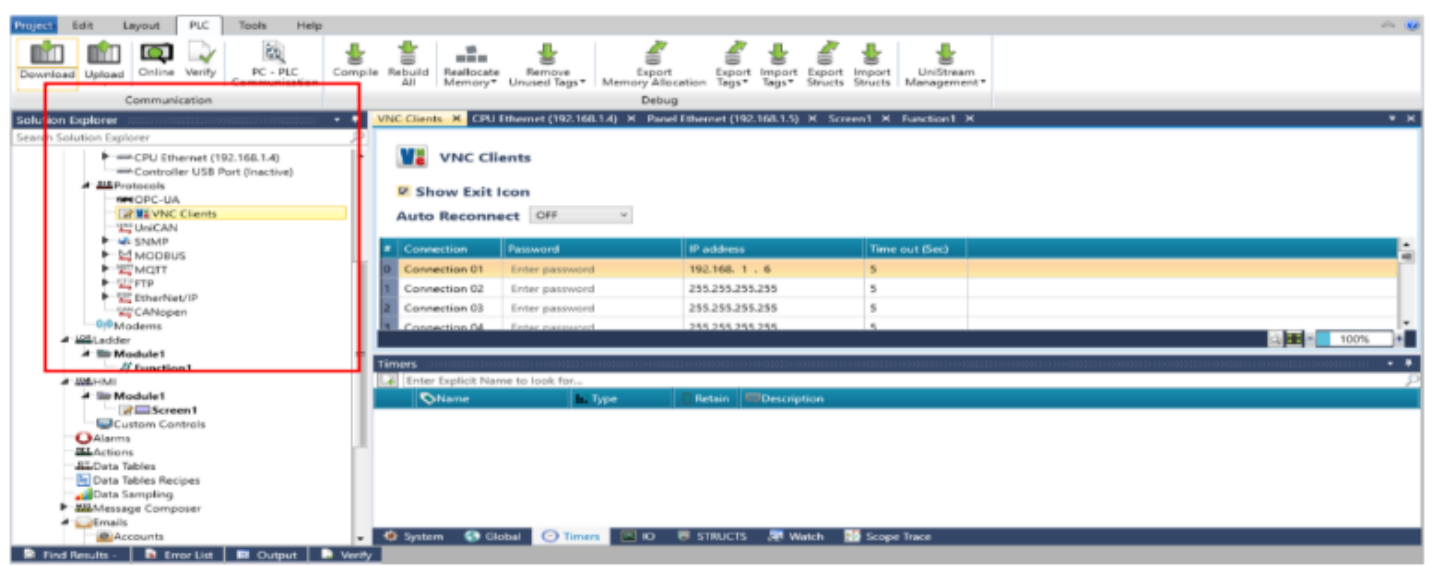

Figure 32 Unitronics PLC VNC IP 


\section{Configuring I/Os}

Systems integration involves connecting different subsystems into a more extensive system and ensuring synchronous function. The subsystems transmit data through inputs/outputs (I/Os) using either hardwired connections or a communication protocol like EthernetIP.

\subsection{Configuring I/Os on the Robots}

The robot I/Os are configured for two-way data transmission with the master PLC over EthernetIP by exchanging digital handshakes as binary inputs and binary outputs. The inputs from the master PLC help execute the programs on the robots, and the outputs from the robots give the status feedback to the master PLC. Subsystems that don't support communication over EthernetIP are controlled using hardwired signals from the robot's I/O boards.

\subsubsection{Setting EthernetIP I/Os on the robots}

The master PLC controls the enable, hold, fault reset and program selection functions of the robots through the User Operator Panel (UOP) I/Os.

On FANUC robots, the UOP I/Os can be configured by following the steps below.

1. Press the IO button on the teach pendant. A window will open, shown in Figure 33.

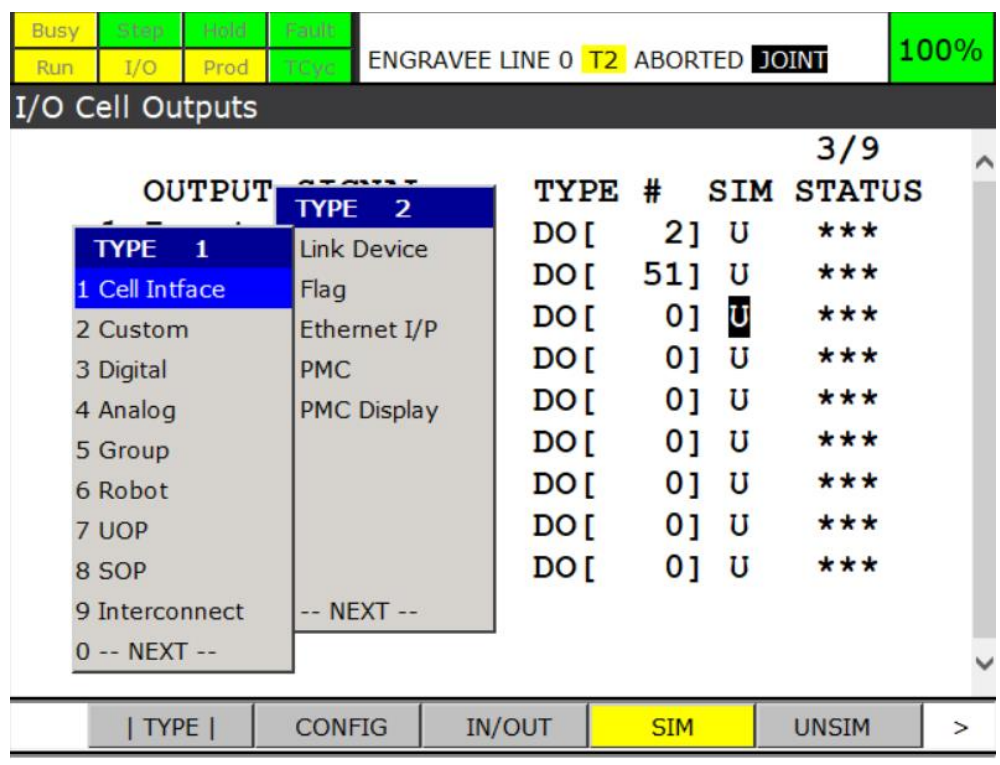

Figure 33 I/O configuration menu on FANUC robots

2. Press F1 to open the [TYPE] menu and select UOP. 


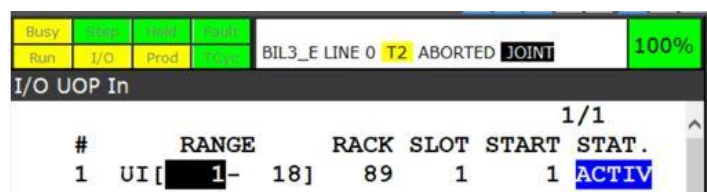

\begin{tabular}{|l|l|l|l|l} 
[ TYPE ] & MONITOR & IN/OUT & DELETE & \% HELP \\
\hline
\end{tabular}

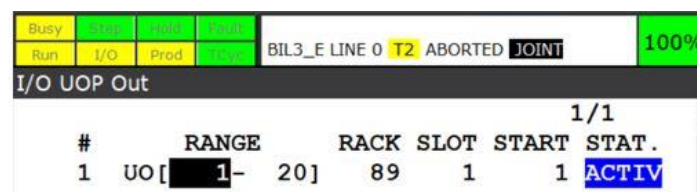

\begin{tabular}{l|l|l|l|l|l}
\hline [TYPE ] & MONITOR & IN/OUT & DELETE & भै HELP \\
\hline
\end{tabular}

Figure 34 UOP I/O configuration

3. Select [F3 CONFIG] menu a window opens shown in Figure 34

4. Enter the values in RANGE, RACK, SLOT, START columns shown in Figure 34. A RACK value of 89 connects the I/Os over Ethernet with the master PLC.

5. START value indicates the bit address one to start the UI

6. Switch between UOP inputs and UOP outputs by pressing [F3 IN/OUT]

Although UOP I/Os control the robots from the master PLC, they cannot be used as handshakes to indicate the progress of a program or control any hardware. FANUC robots use digital I/Os to confirm the execution of a program segment or for controlling I/Os on the master PLC or the robot.

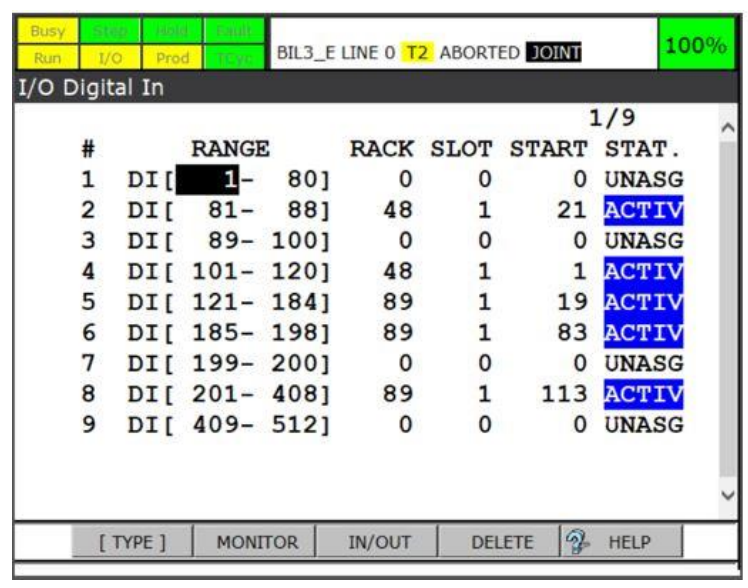

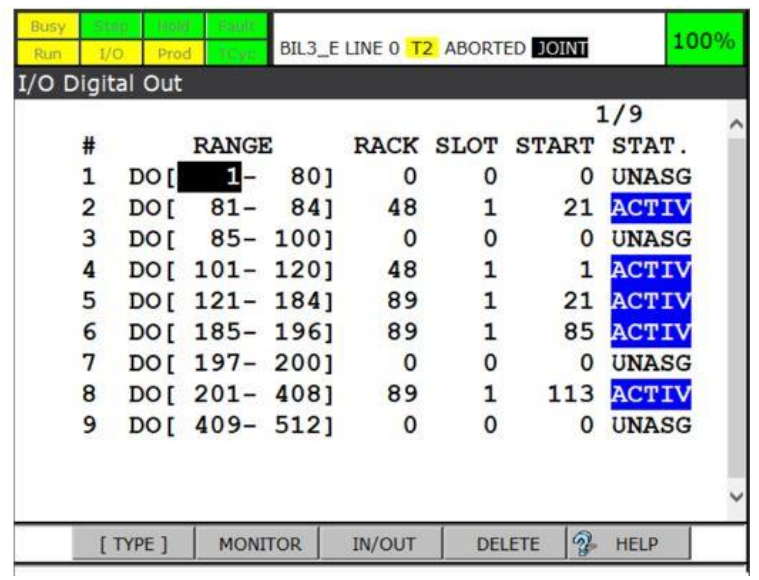

Figure 35 Digital I/O configuration

The digital I/O setup can be done by following the steps below,

1. From the TYPE menu, select Digital as shown in Figure 33 
2. Enter the values in RANGE, RACK, SLOT, START columns as shown in Figure 35Figure 35. A RACK value of 89 connects I/Os over EthernetIP, and a RACK value of 48 connects the I/Os on the robot controller I/O board

3. The START value is 19 for DI[101-120] and 21 for DO[121-184] because UOP I/Os occupy the first 18 digital inputs and the first 20 digital outputs

4. The START for the next DI and DO ranges is set to account for the bit length of the word of the previous ranges

5. Switch between digital inputs and digital outputs by pressing [F3 IN/OUT]

\subsubsection{Setting hardwired I/Os on the robots}

Hardwired I/Os are input and outputs which transmit digital or analog signals using physical cables between systems. Hardwired I/Os are used on Robot 3 to control the quick tool changers. The wiring scheme for the tool changers is shown in Figure 36.

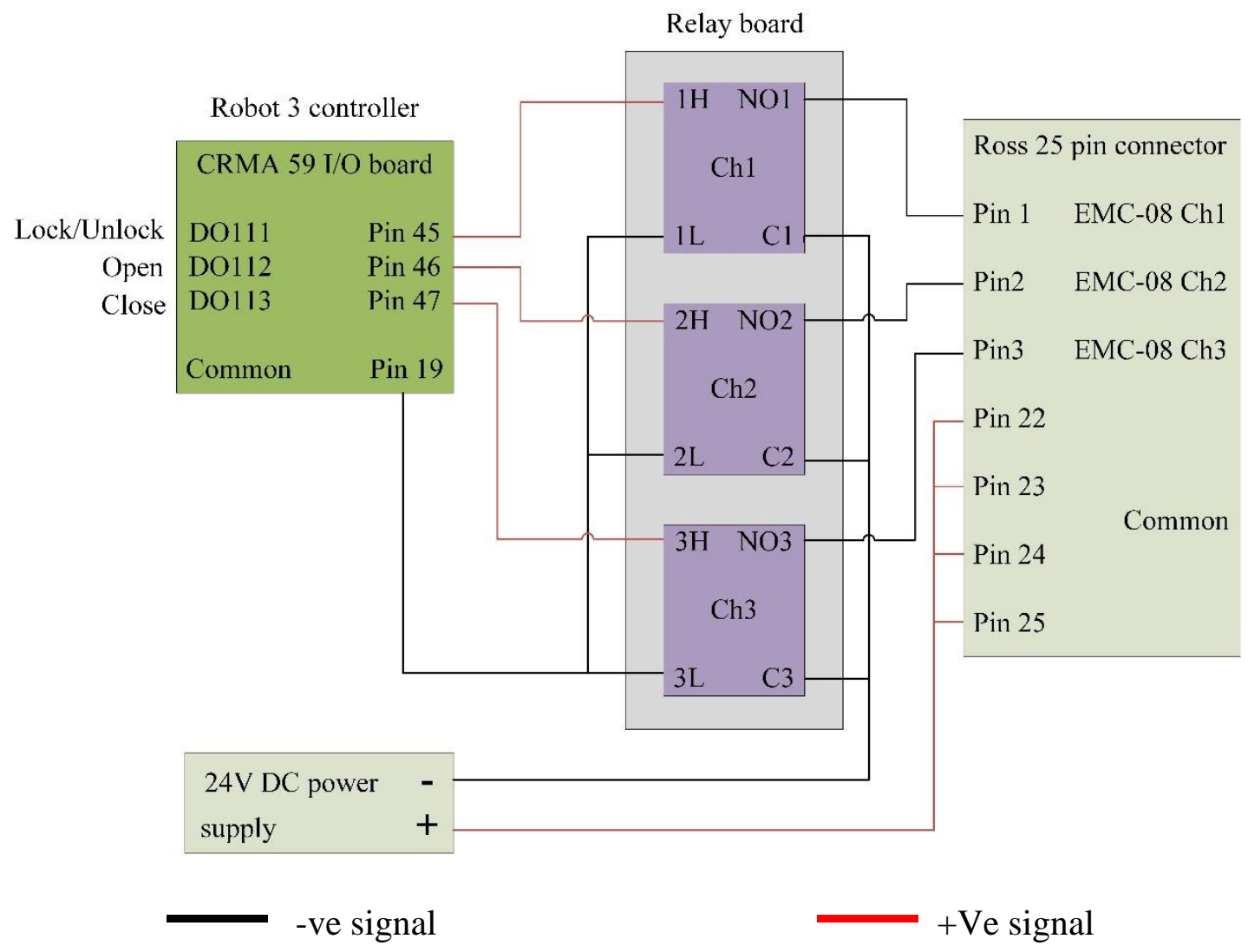

Figure 36 Wiring scheme for quick disconnect grippers

Robot 3s digital outputs are wired to the robot CRMA59 I/O board on pin nos. 45, 46, 47, and pin 19 as a signal ground/common. These pins are linked to digital output channel numbers DO111, DO112, DO113 for lock/unlock, open and close function on the teach pendant. These channels on Robot 3 control the relay coils on the relay board, and a 24VDC signal is passed to the Ross 25 pin connector via the relay contacts. 


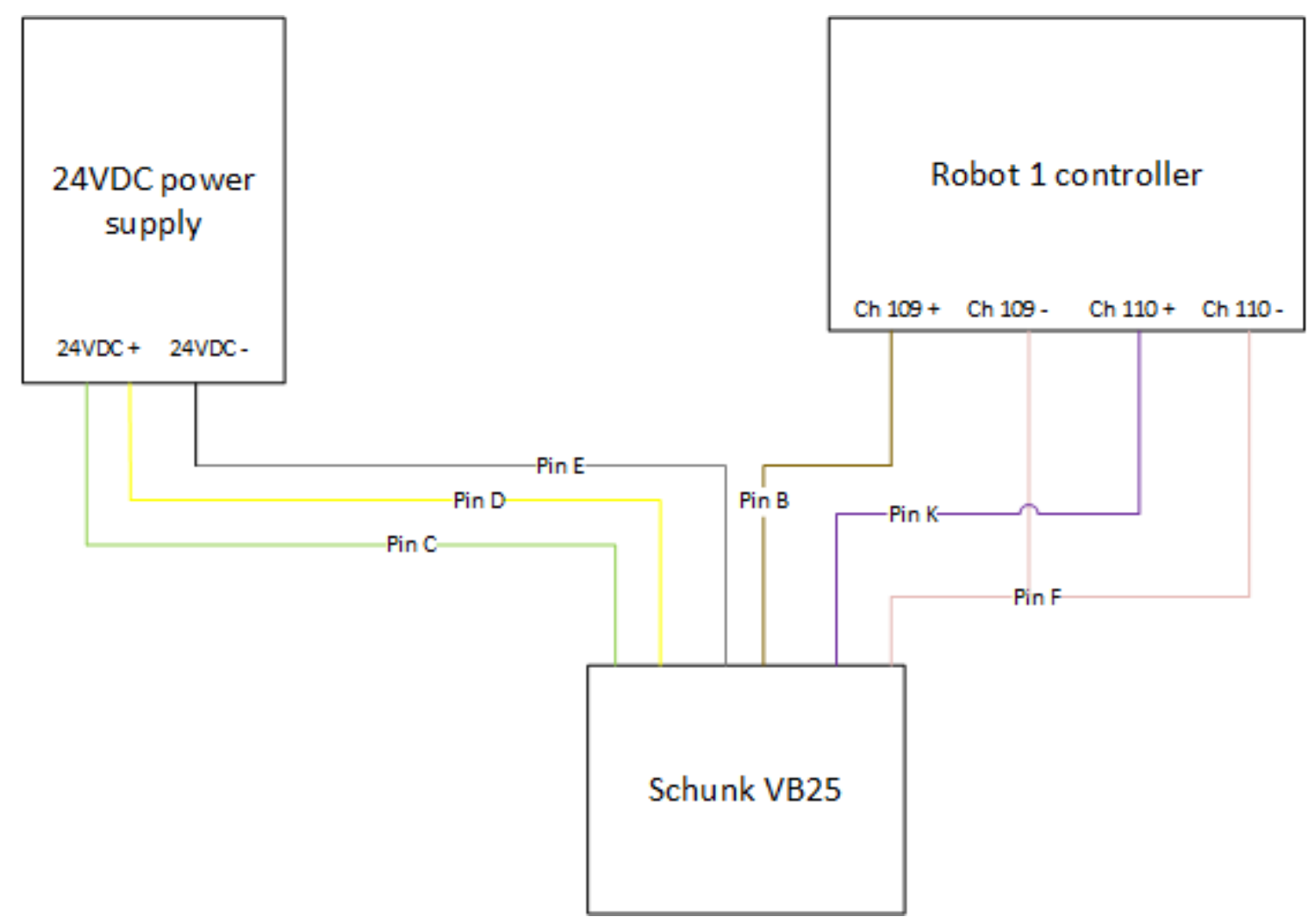

Figure 37 Schunk VB25 wiring scheme

Robot 1 uses a vacuum gripper as the end of the arm tooling with a Schunk Ventiblock VB25 vacuum manifold controlling the suction. The wiring scheme for the manifold is as shown in Figure 37. A 24VDC power supply powers the manifold, and Robot 1 uses a twelve-pin circular connector to control the manifold.

\subsection{Configuring Donald Engineering station I/Os}

The master PLC controls the Donald Engineering station with the Unitronics PLC configured to accept control signals from the master PLC over EthernetIP.

To configure I/Os over EthernetIP, the Unitronics PLC is set as an adapter to accept commands and the master PLC as a scanner to give commands by following the steps below.

1. Open Unilogic programming software and create a new project with the hardware on the Donald Engineering station.

2. Access Structs through the global database window. Create EIP_input_struct and EIP_output_struct having 16 bits each to read/write information, as shown in Figure 38 


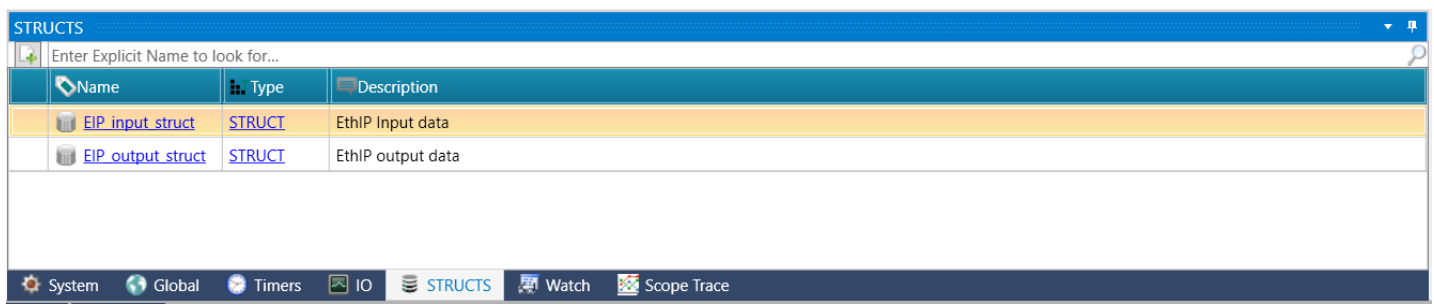

Figure 38 Unitronics Struct configuration

3. Go to Project $>$ PLC communications $>$ Protocols $>$ Ethernet IP $>$ Adapter $>$ Add new adapter node to access adapter settings and enter the values as shown in Figure 39.

Add New Adapter Node

\begin{tabular}{|c|c|c|c|c|c|c|}
\hline Node Name & O2T Assembly Ins & Input & Input Size (Bytes) & T20 Assembly Ins & Output & Output Size (Byte \\
\hline AB_PLC & 100 & EIP_Input & 2 & 101 & EIP_output & 2 \\
\hline
\end{tabular}

Figure 39 Unitronics adapter settings

4. Commands from the master PLC are written in the EIP_input_struct, and commands from the Unitronics PLC are transmitted to the master PLC using the EIP_Output_struct.

\subsection{Configuring the master PLC I/Os}

The Rockwell Automation Allen Bradley L32E PLC is the master controller in the system that manages inputs from the subsystems and controls the process. The PLC uses I/Os configured on EthernetIP and hardwire connections to fetch data from all subsystems.

\subsubsection{Setting EthernetIP I/Os on the master PLC}

The master PLC uses I/Os over EthernetIP to control Robots 1,2,3, and the Donald Engineering station by reading and writing data into data registers. The data registers are configured on RSLogix5000 programming software using generic ethernet modules for each device, as shown in Figure 40. Generic ethernet modules are configured by following the steps below,

1. Open RSLgoix 5000 software 
2. From the Controller organizer window, right-click on Ethernet as shown in Figure 41 and select the new module option. Search for generic ethernet module and click OK to open a window shown in Figure 40

3. Enter the Name, IP address, and input, output data register size for Robot 1, Robot 2, Robot 3, and the Donald Engineering station as shown in Figure 40. The data register size on the master PLC matches the register size on each subsystem.
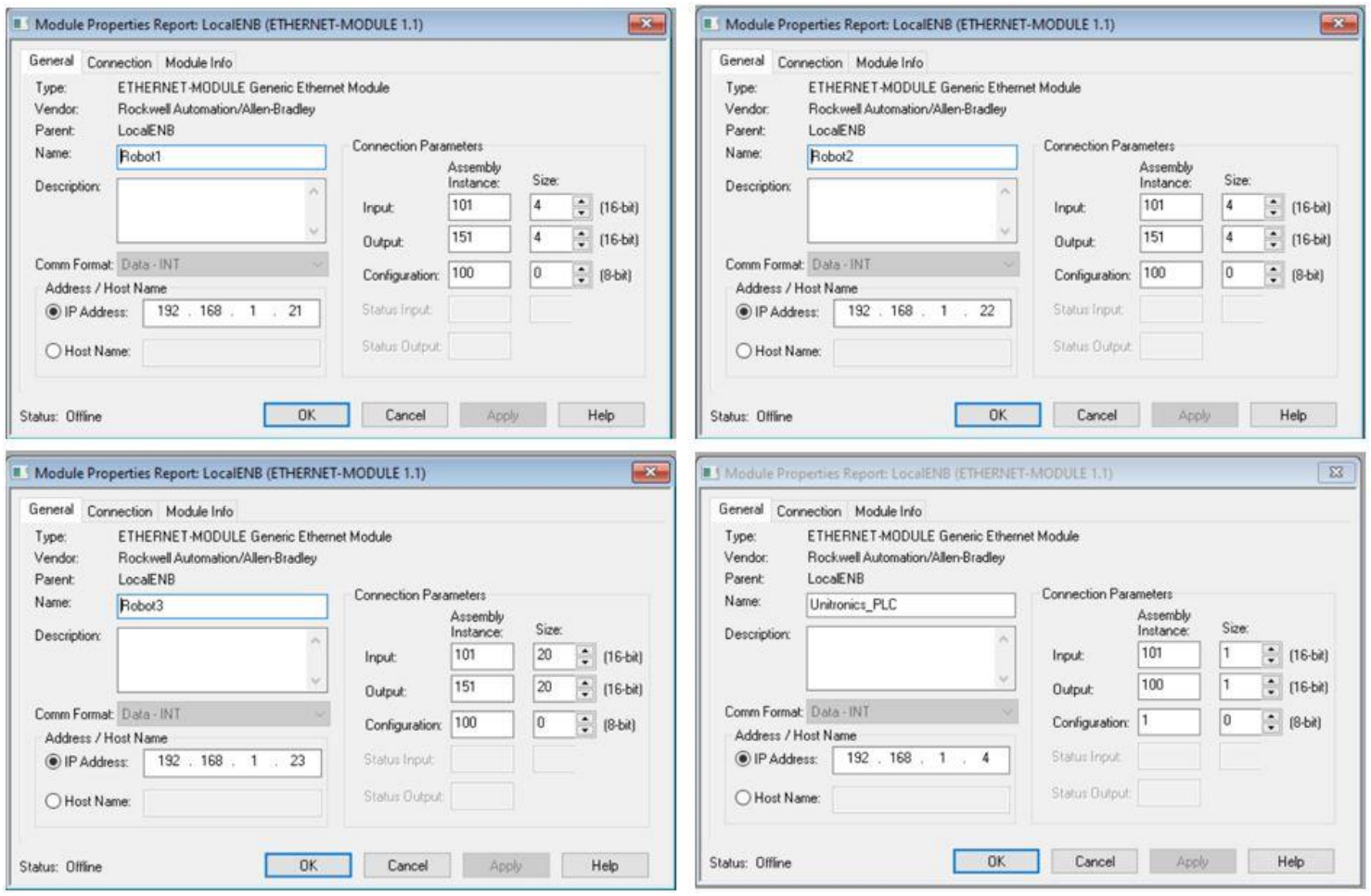

Figure 40 Generic ethernet modules for devices

4. After entering desired values, click OK to finalize settings and download the program to the master PLC

5. After a successful configuration, the devices show up on the device tree shown in Figure 41

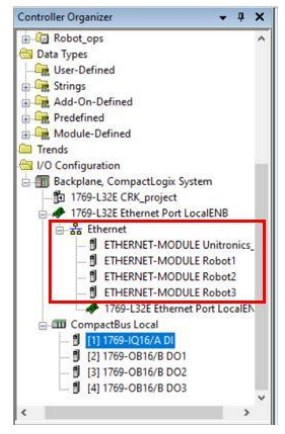

Figure 41 Generic ethernet module in the device tree 


\subsubsection{Setting hardwired I/Os on the master PLC}

The master PLC uses hardwired I/Os to control the belt conveyor, Vacuum, Dremel tooling and get feedback from the photoelectric sensors and the inductive proximity switches. The hardwired I/Os use PLC I/O cards to transmit the +24VDC control command or receive +24VDC feedbacks. The PLC has a 1769-IQ16 digital input card and three 1769-OB16 digital output cards from Rockwell Automation's compact I/O series, which attach to the side of the PLC, as shown in Figure 42.

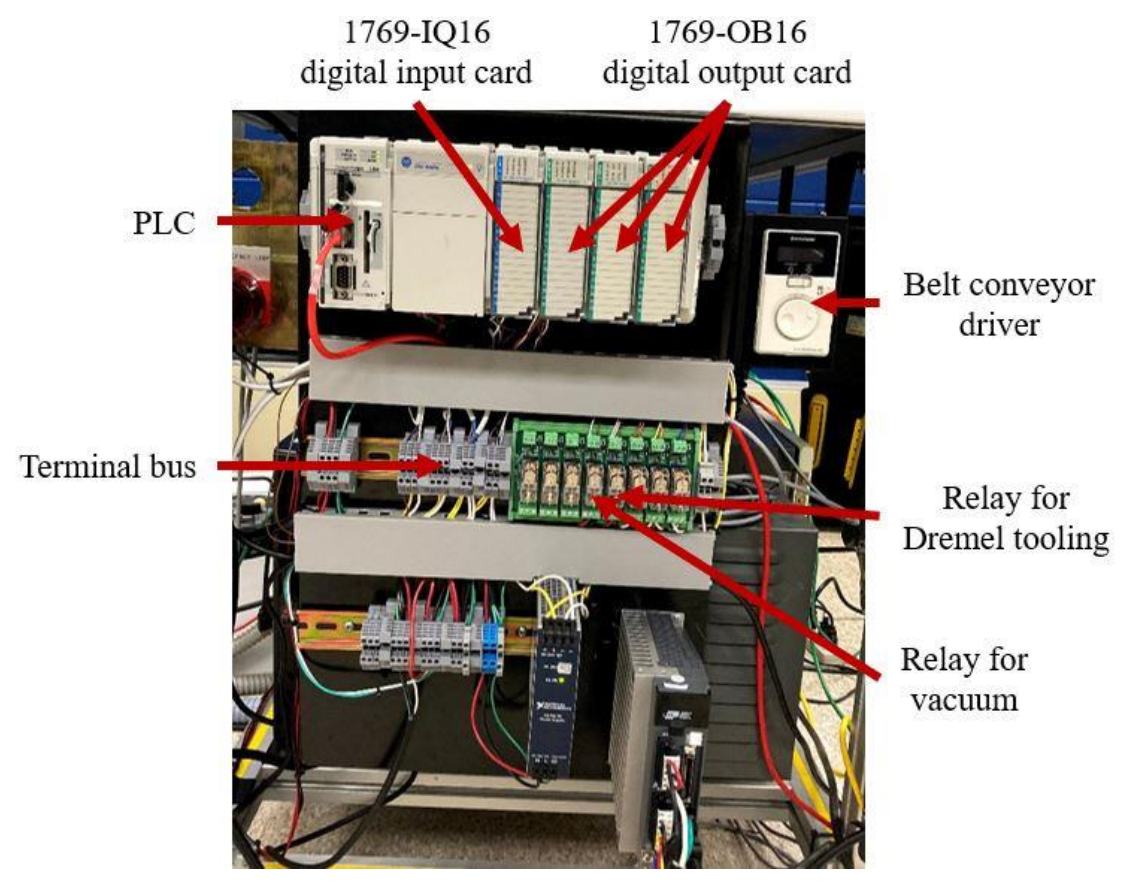

Figure 42 Master PLC hardwired I/O specification

The photoelectric sensors and inductive proximity switches are wired to the digital input card via the terminal bus. The relays controlling the Dremel tooling and vacuum, the belt conveyor driver are all wired to the first digital output card. Figure 43 shows the channel configuration for the hardwire I/O cards on the master PLC.
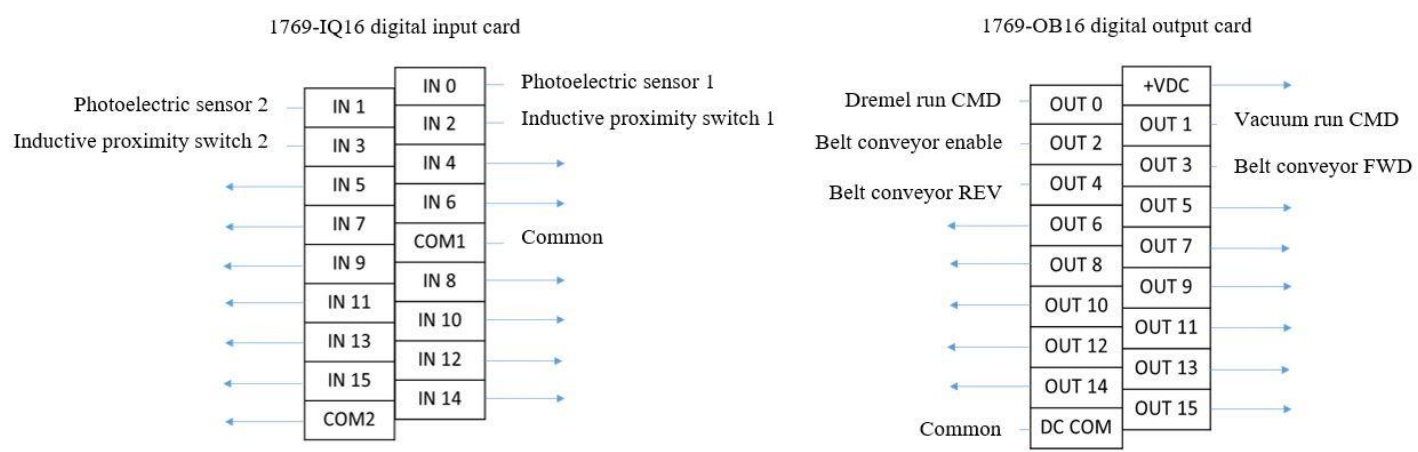

Figure 43 Master PLC I/O card channel configuration 


\section{Programming the subsystems}

The previous chapters cover hardware configuration for the process and the communication settings to transfer data between the subsystems. This chapter covers the programming of the subsystem to follow a series of software-coded instructions to control the process.

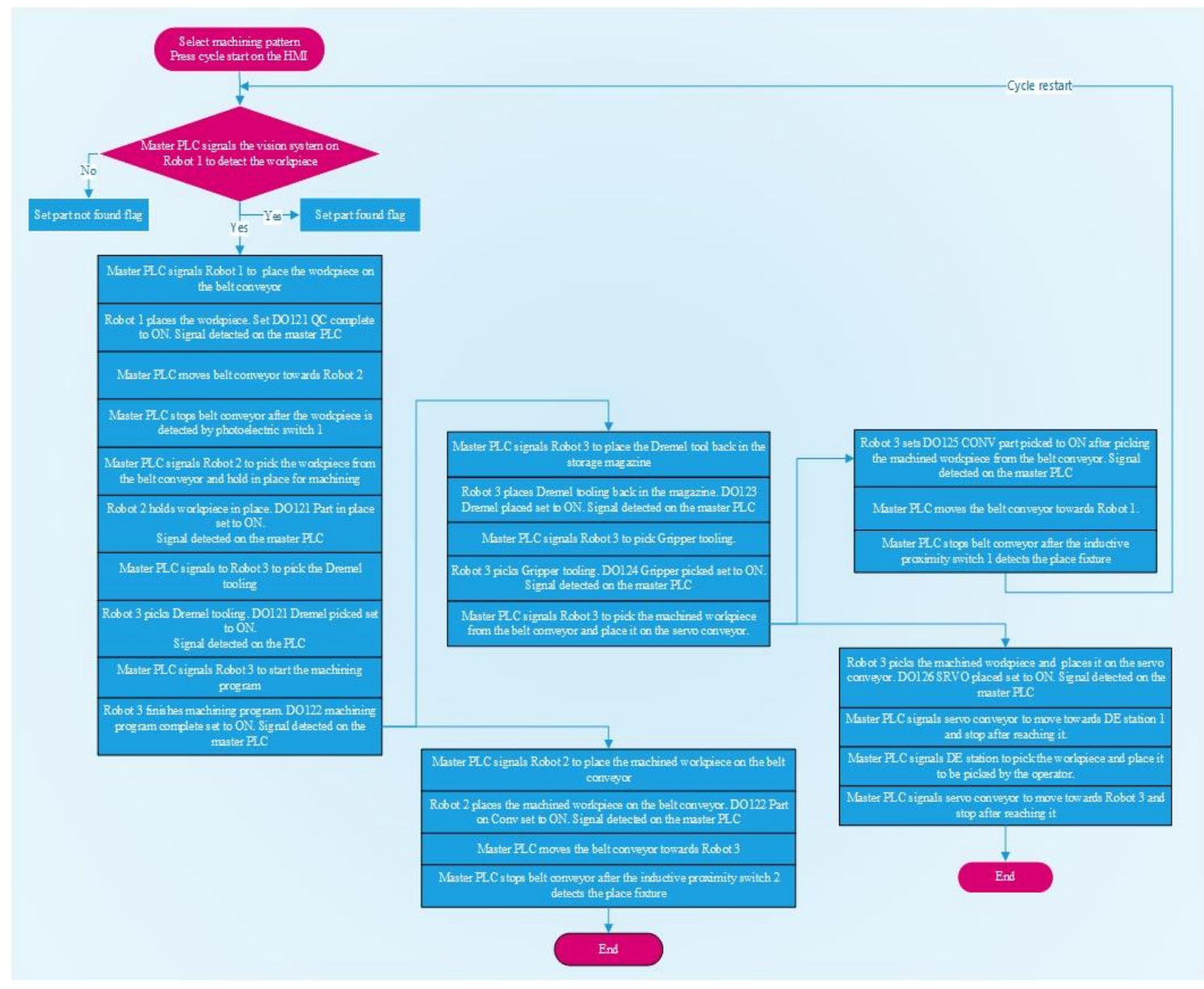

Figure 44 Process algorithm

Subsystems Robots 1,2,3 and the Donald engineering stations are programmable and store programs that follow the process algorithm shown in Figure 44. The master PLC calls programs on the subsystems over EthernetIP depending on the I/O status to follow the process algorithm.

\subsection{Programming of the Robots}

The program on the robots follows a set of motion instructions to move the robots. The FANUC robots are programmed with a teach pendant or FANUC Roboguide simulation software. 


\subsubsection{Robot 1}

Robot 1 performs a quality check using the FANUC 2D iRVision system to detect the correct workpiece and is programmed using the teach pendant according to the algorithm shown in Figure 45.

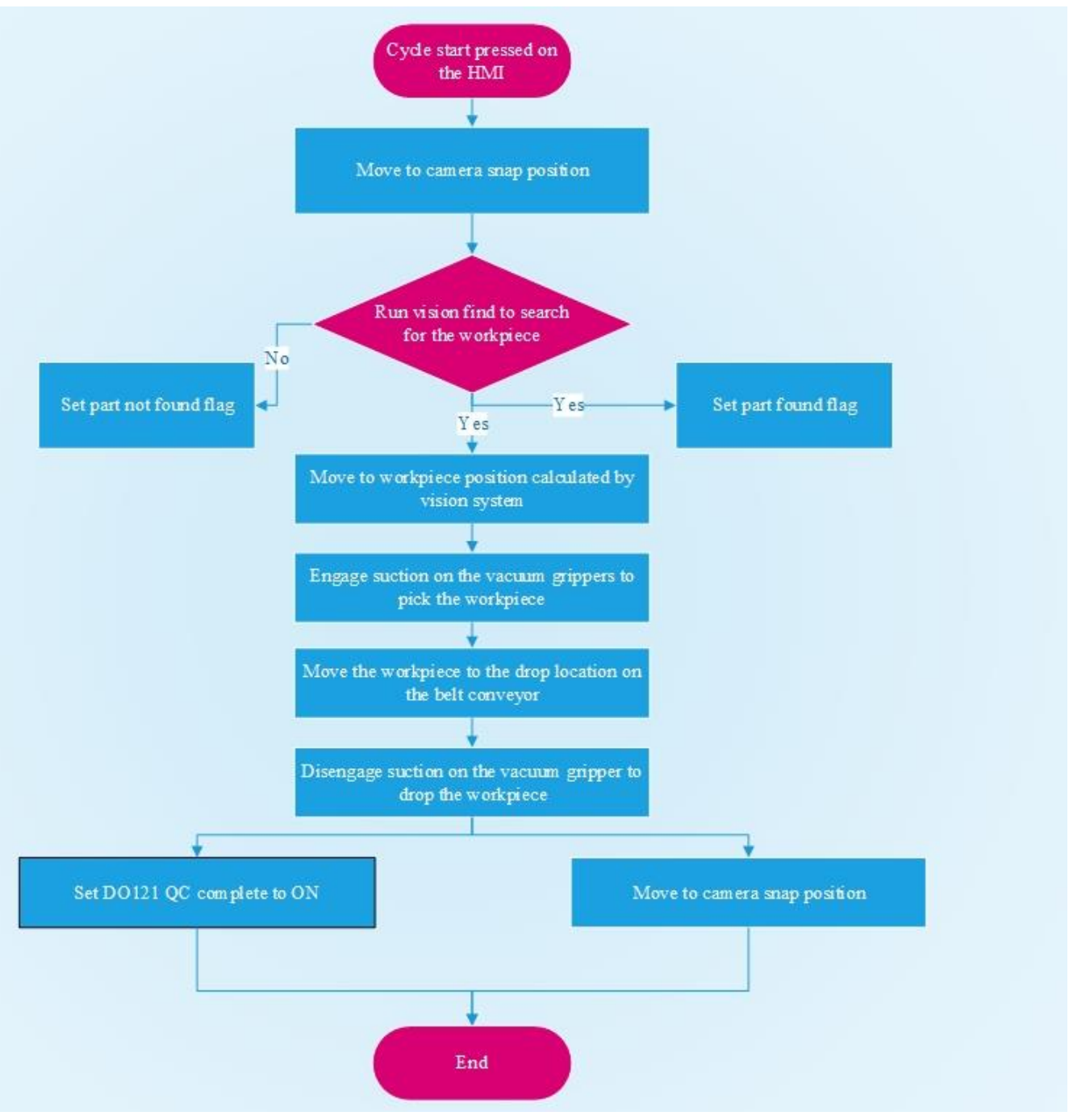

Figure 45 Robot 1 program algorithm

When the cycle start push button is pressed on the HMI, the master PLC signals Robot 1 to execute the program called PNS0001, which signals the vision system to find the workpiece. If the workpiece dimensions are a ninety-eight percent match to the desired length and width of the workpiece, the workpiece is placed on the belt conveyor.

A workpiece with dimensions below ninety-eight percent of the expected value is not picked, and the operator has to manually replace it with another workpiece. After placing the workpiece on the belt conveyor, Robot 1 sets DO121 ON, and the master PLC commands the belt conveyor to move towards Robot 2 . 


\subsubsection{Robot 2}

Robot 2 picks the workpiece, holds it in place for machining, and drops the workpiece back on the belt conveyor after the machining process is completed.

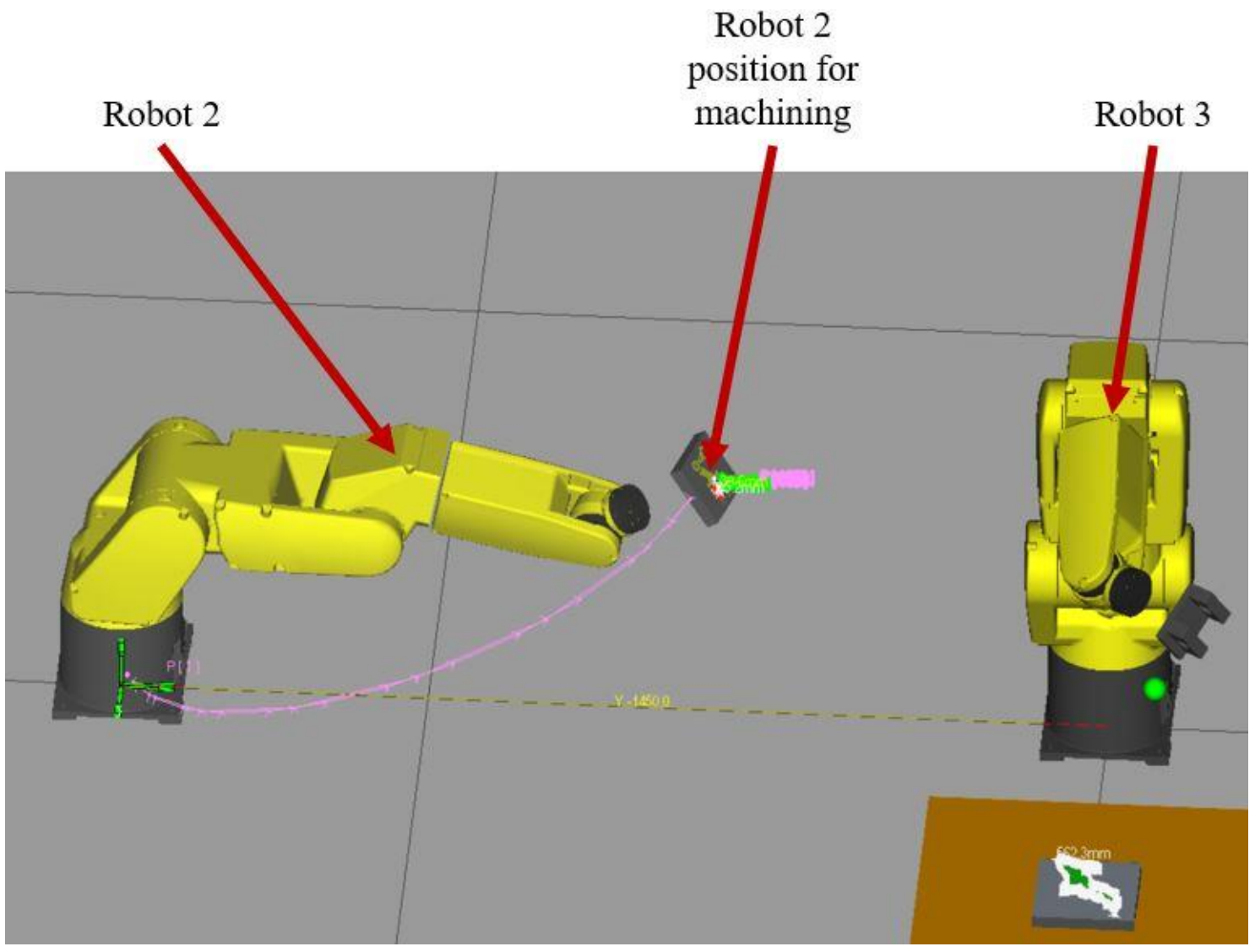

Figure 46 FANUC Roboguide showing Robot 2s position

Robot 2 is programmed using the teach pendant and has the positions calculated using FANUC Roboguide software shown in Figure 46. The positions at which Robot 2 holds the workpiece for machining are calculated in Roboguide. Robot 3 uses these positions to orient the Dremel tooling to the surface of the workpiece for generating the machining program.

After the positions are calculated, Robot 2 is programmed according to the algorithm shown in Figure 47. The master PLC selects the program and signals Robot 2 to execute the selected program. The master PLC selects PNS0001 on Robot 2 to pick the workpiece from the belt conveyor and hold it in the calculated position to be machined by Robot 3 . Robot 2 sets DO121 to ON to signal the workpiece is in place to the master PLC which starts the machining program on Robot 3. 


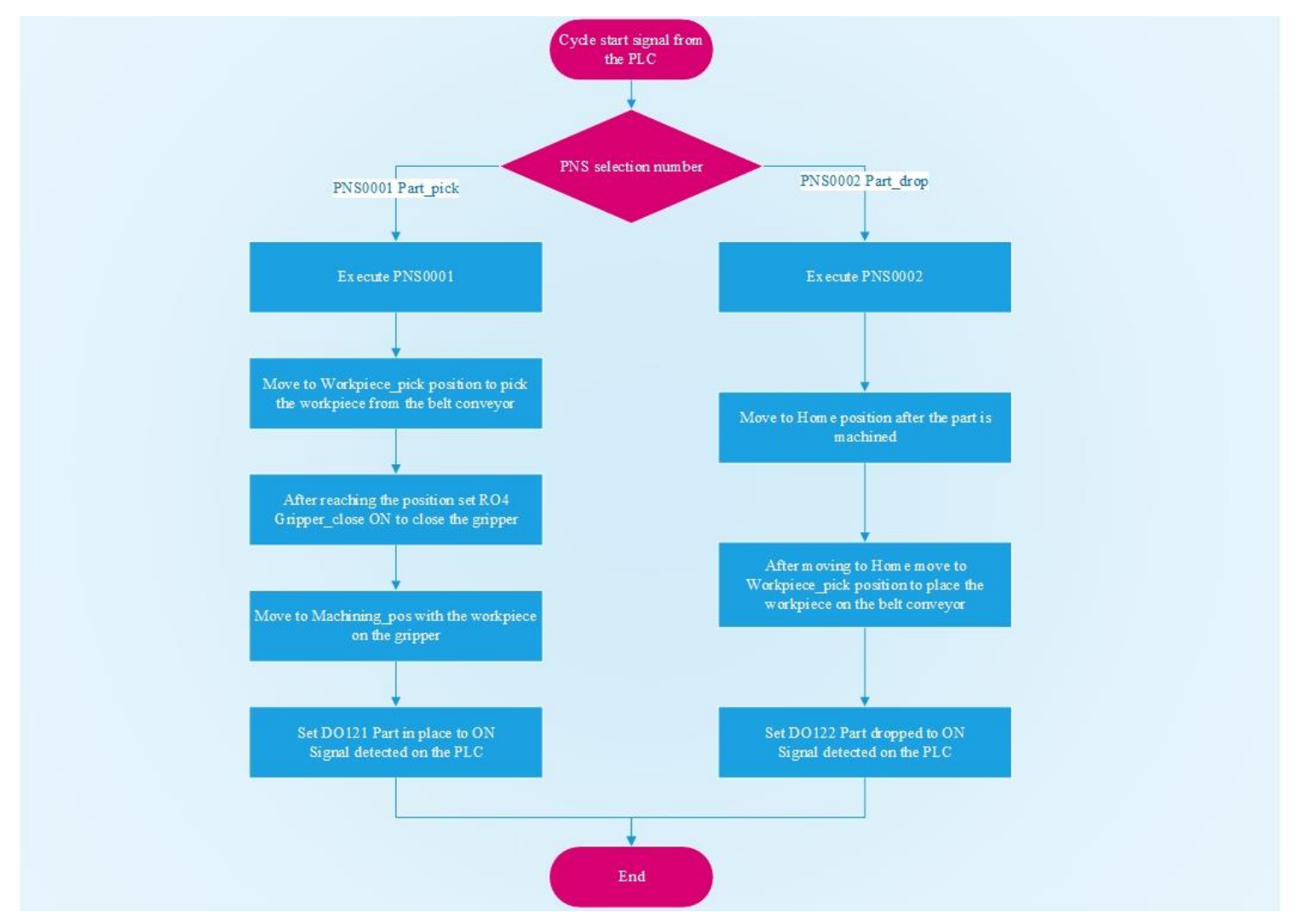

Figure 47 Robot 2 program algorithm

After the workpiece is machined, the master PLC selects PNS0002 to drop the workpiece back on the belt conveyor. Robot 2 sets DO122 to ON to signal the master PLC to move the belt conveyor to Robot 3 .

\subsubsection{Robot 3}

When working as a part of the machining process, Robot 3 uses the algorithm shown in Figure 48. The master PLC selects to execute a program from the seven available programs depending on the process shown in Figure 44. After executing each program, Robot 3 sets a digital output to $\mathrm{ON}$ to confirm the completion of the program to the master PLC to schedule the next program in the process. 


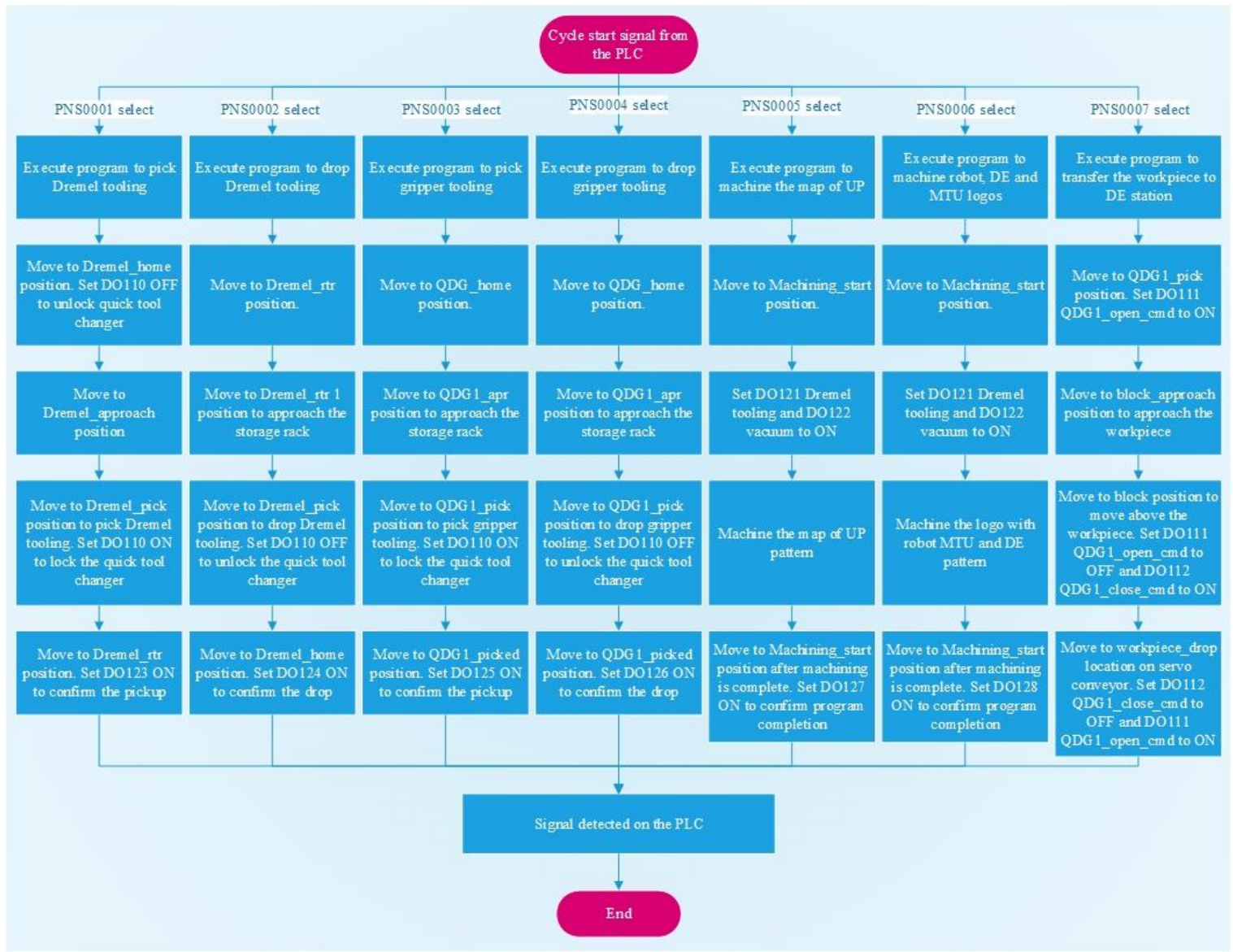

Figure 48 Robot 3 program algorithm

Robot 3 machines one of the two available patterns on the workpiece. The operator selects the desired pattern from the HMI before starting the machining process. The patterns are coded into Robot 3 using FANUC Rogoguide simulation software. The program path is created using the CAD to path feature on Roboguide, which creates motion instructions for the robot using outlines drawn on a CAD model shown in Figure 49.

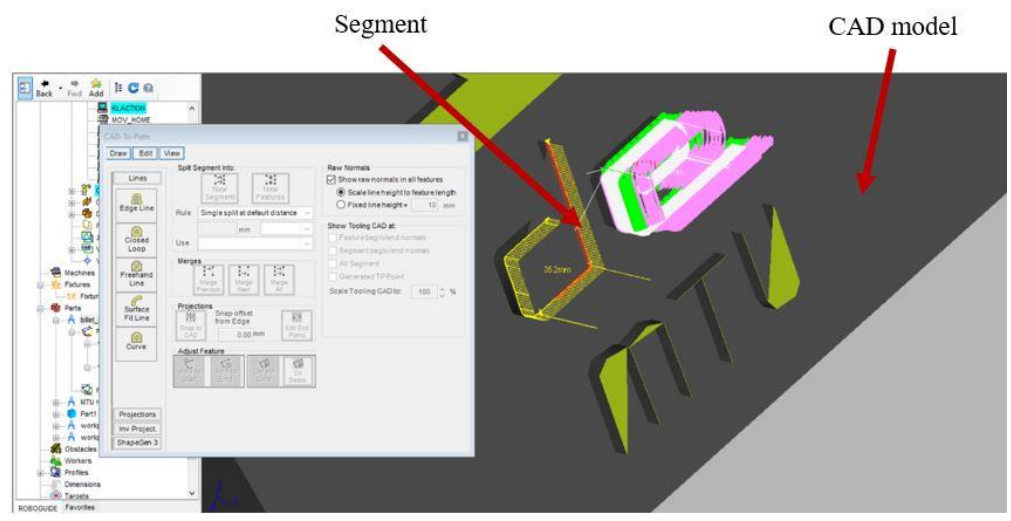

Figure 49 CAD to path on Roboguide 
After importing a CAD STEP file in Roboguide, a feature outline is drawn as a segment on the CAD model using the edge line option shown in Figure 49. After outlining the desired features, the segment is programmed into a path using program settings on the segment property window shown in Figure 50.

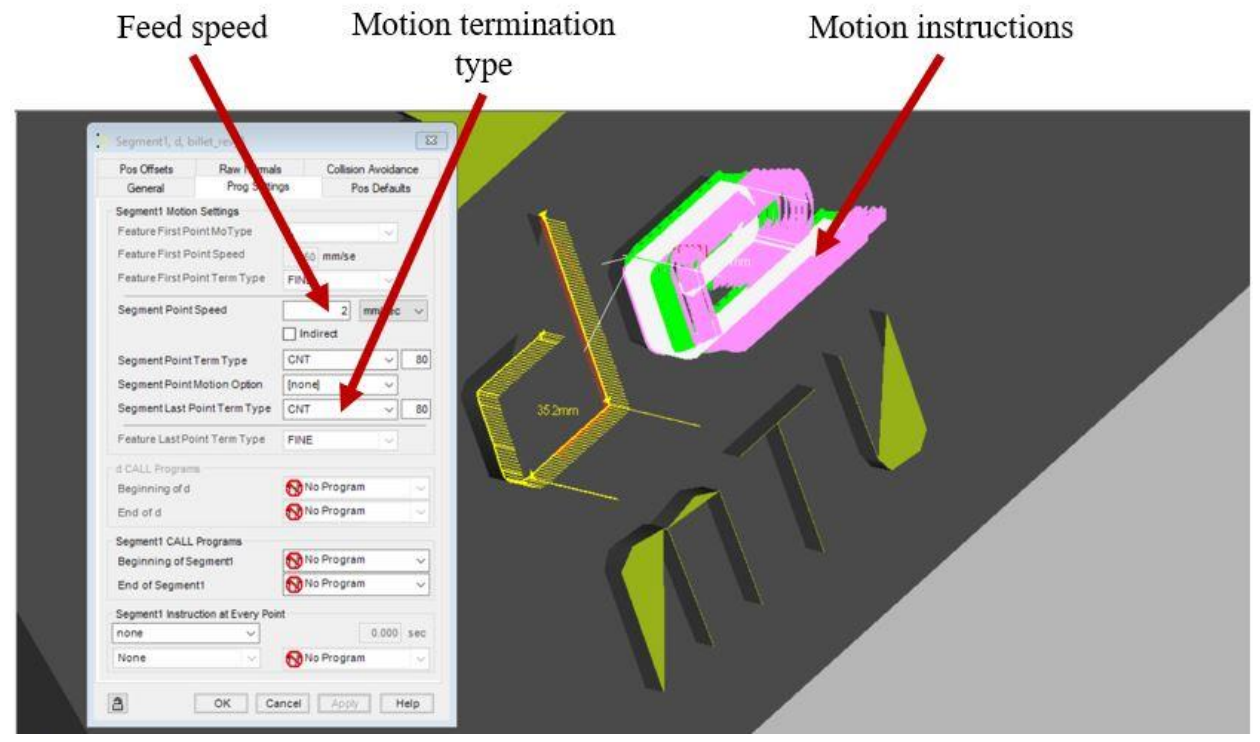

Figure 50 Segment programming

The feed speed is $2 \mathrm{~mm} / \mathrm{sec}$, and the motion termination type is CNT80 to get the best results. After the program settings are done, the tool orientation and path generation are set through the pos default menu. The tool is oriented to move along the -X-axis and perpendicular to -Z-axis, and the points are programmed to be generated every $0.250 \mathrm{~mm}$ shown in Figure 51.

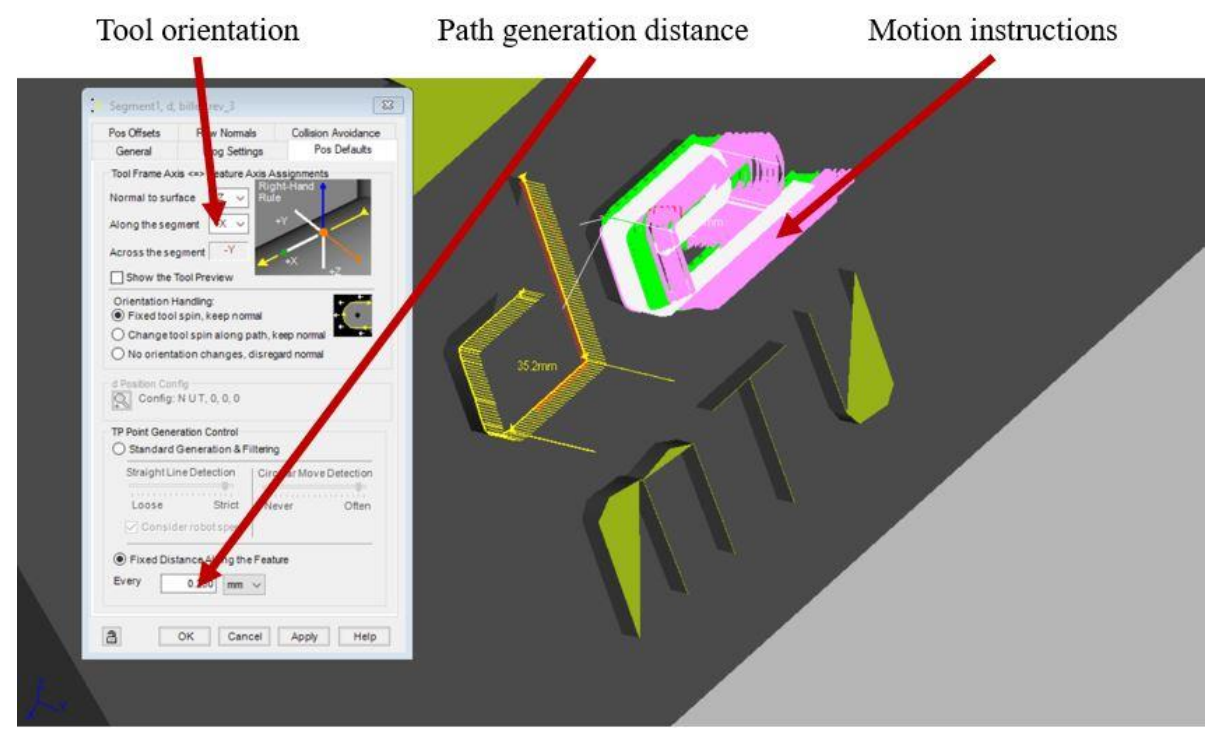

Figure 51 Tool orientation for Roboguide 
Roboguide uses Robot Neighbourhood to download offline programs and simulations to the physical robot over Ethernet. After the program is created on Roboguide, the Robot Neighbourhood is configured to read the target IP address of Robot 3, shown in Figure 52.

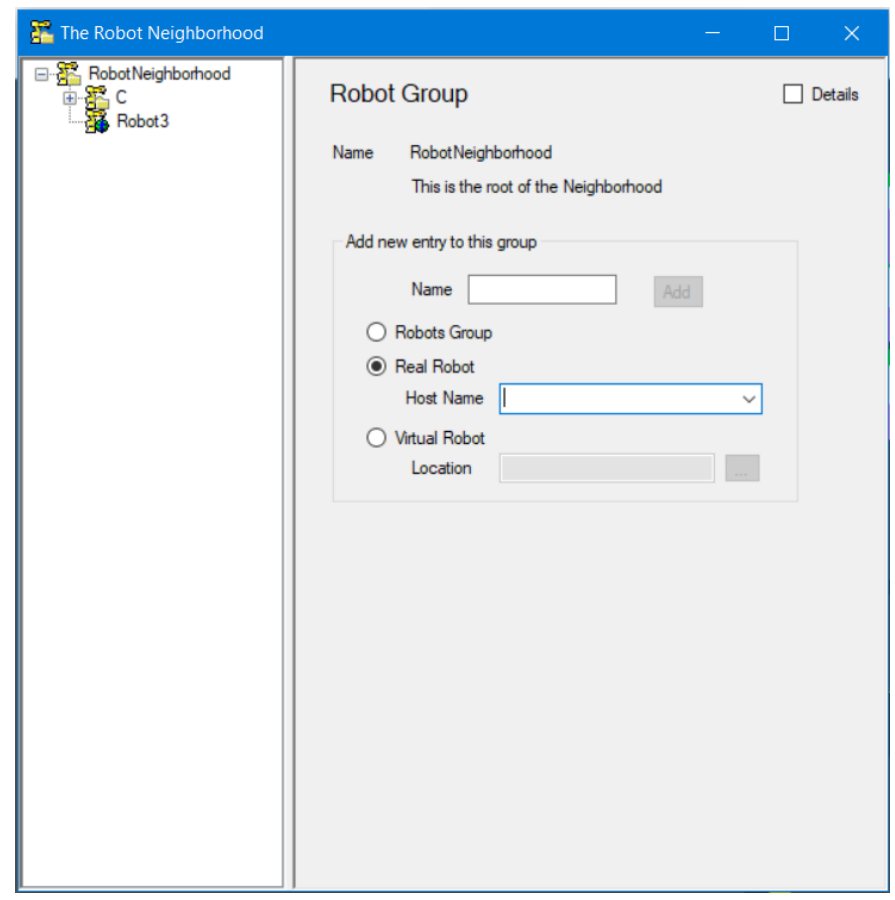

Figure 52 Robot neighborhood

After the robot IP is added the program is downloaded by going to Roboguide > Toolbar $>$ Project $>$ Export $>$ Edit List $>$ select the program.

After selecting the program it can be downloaded through Project $>$ Export $>$ To Robot $>$ Select project file $>$ Select Robot $3>$ Click Export. If the download the successful, the program is seen on the Robot 3 s teach pendant.

\subsection{Donald Engineering station}

The Unitronics PLC and HMI control the Donald Engineering station for independent operation, and the master PLC controls the Donald Engineering station for coordinated operation with the system. The master PLC reads and writes commands to the Unitronics PLC through the EIP_input_struct and EIP_output_struct registers configured in the previous section.

Figure 53 shows the ladder logic for writing position feedbacks from the actuators to the master PLC. The feedback input state is written to a bit on the 16-bit EIP_output_struct, which is read on the master PLC via EthernetIP.

The Donald Engineering station uses ladder logic programming on the Unitronics PLC to control the actuators in local mode with the Unitronics HMI's inputs shown in Figure 54. 


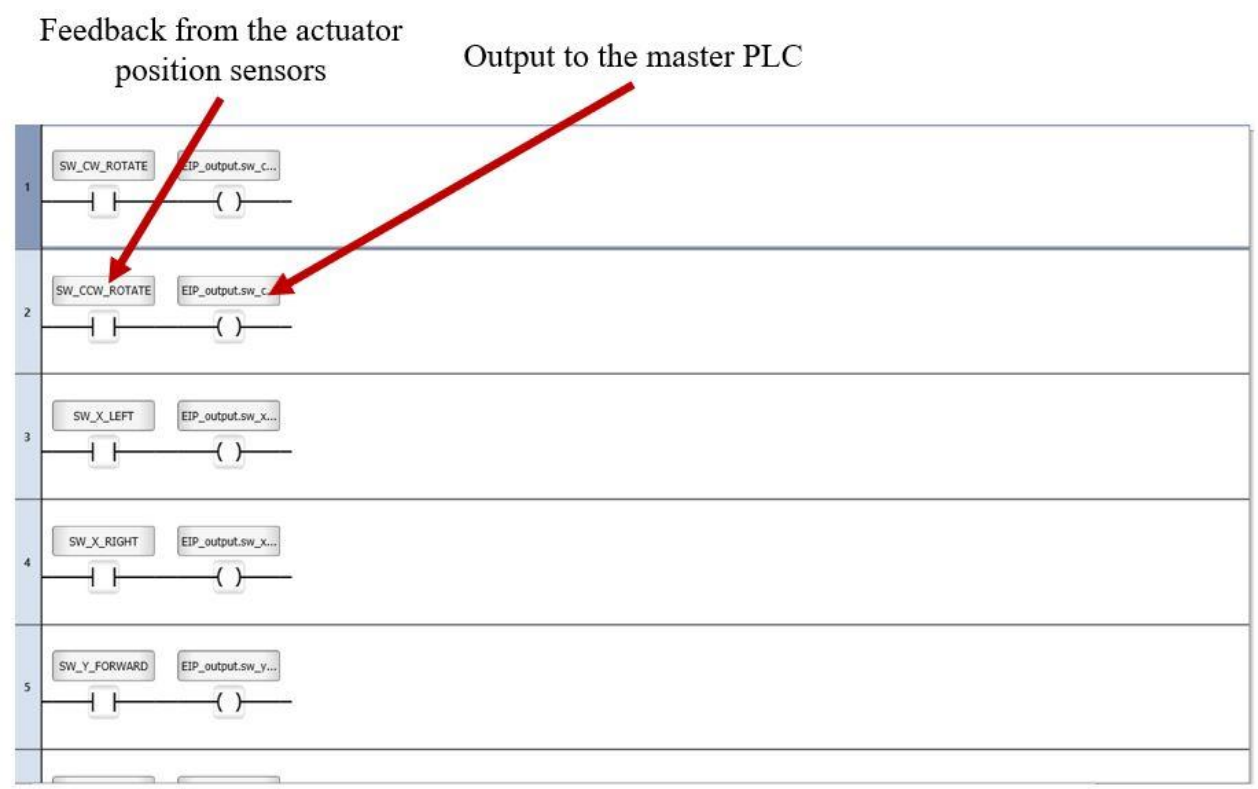

Figure 53 Output commands from Unitronics PLC to the master PLC

The master PLC controls the Donald Engineering station in remote mode through ladder logic by writing commands on the 16-bit EIP_input_struct shown in Figure 54. The commands from the master PLC are interlocked through a remote mode enable bit to inhibit commands from the master PLC when the station is working in local mode.

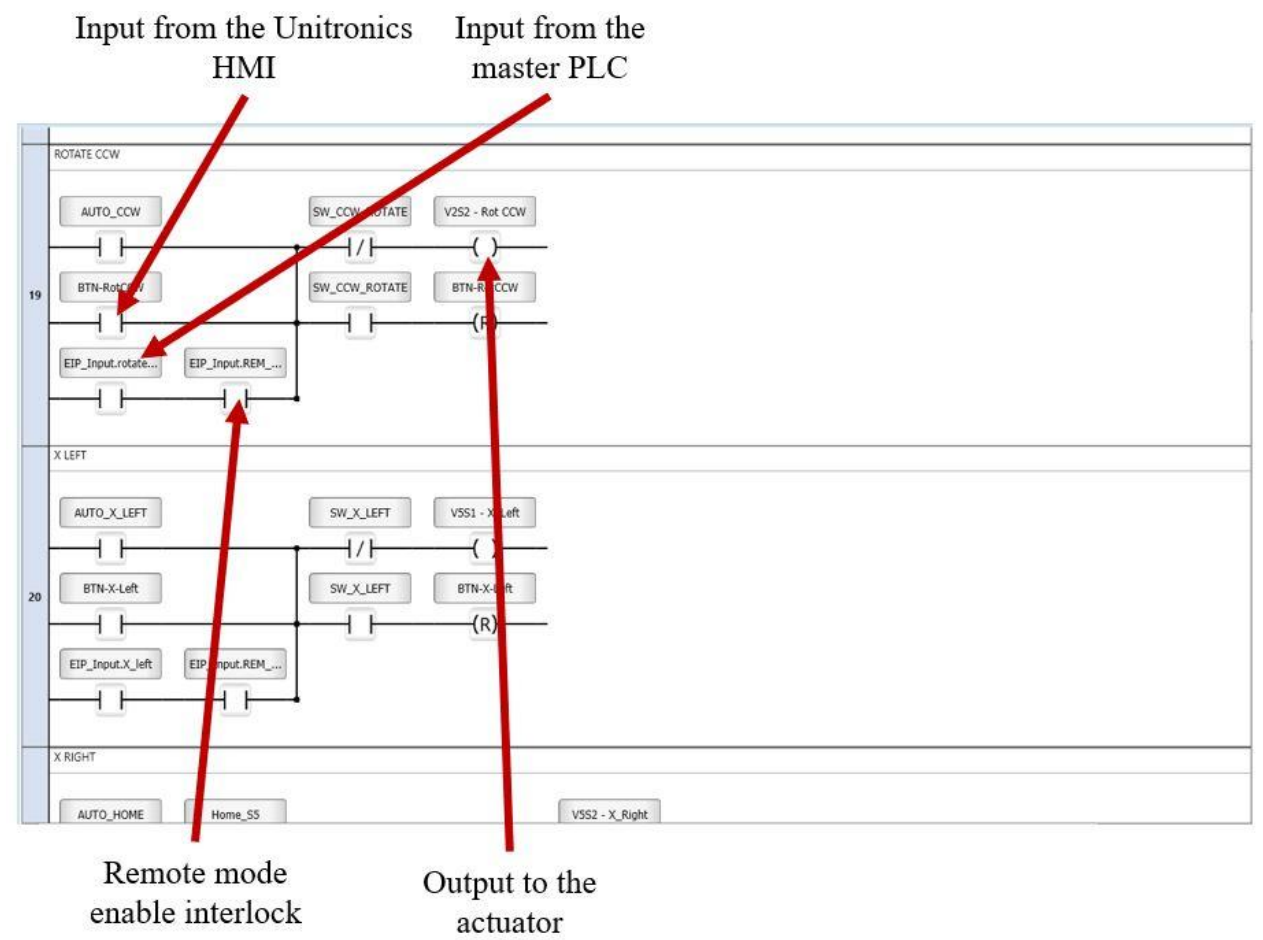

Figure 54 Actuator control logic on the Unitronics PLC 


\subsection{Master PLC}

The master PLC controls Robots 1,2,3, the Donald Engineering station, and the servo conveyor by writing and reading commands over EthernetIP and the belt conveyor using hardwired connections.

Add-On-Instruction is a feature on Rockwell Automation PLCs to program repetitive logic as a standardized control algorithm block. The master PLC uses Add-On-instructions (AOIs) to control the Robots, servo conveyor, and the Donald Engineering station shown in Figure 55.

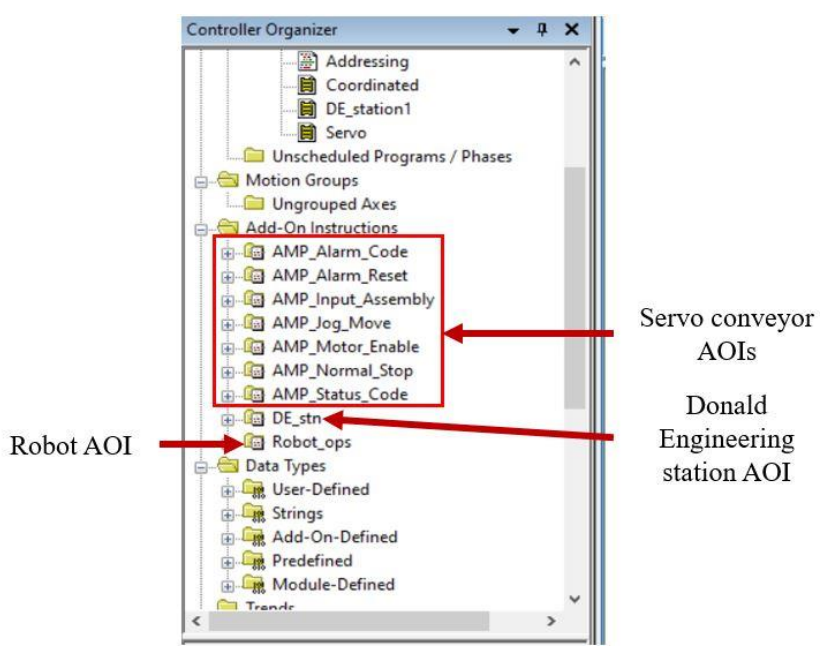

Figure 55 Add-On-Instructions

Robots 1,2,3, being FANUC robots with identical controllers, use the same control algorithm and ladder code for control from the master PLC and is standardized using AddOn-Instructions. Figure 56 shows an AOI block called Robot_ops gets called in the main program and executes the same control algorithm for different robots.

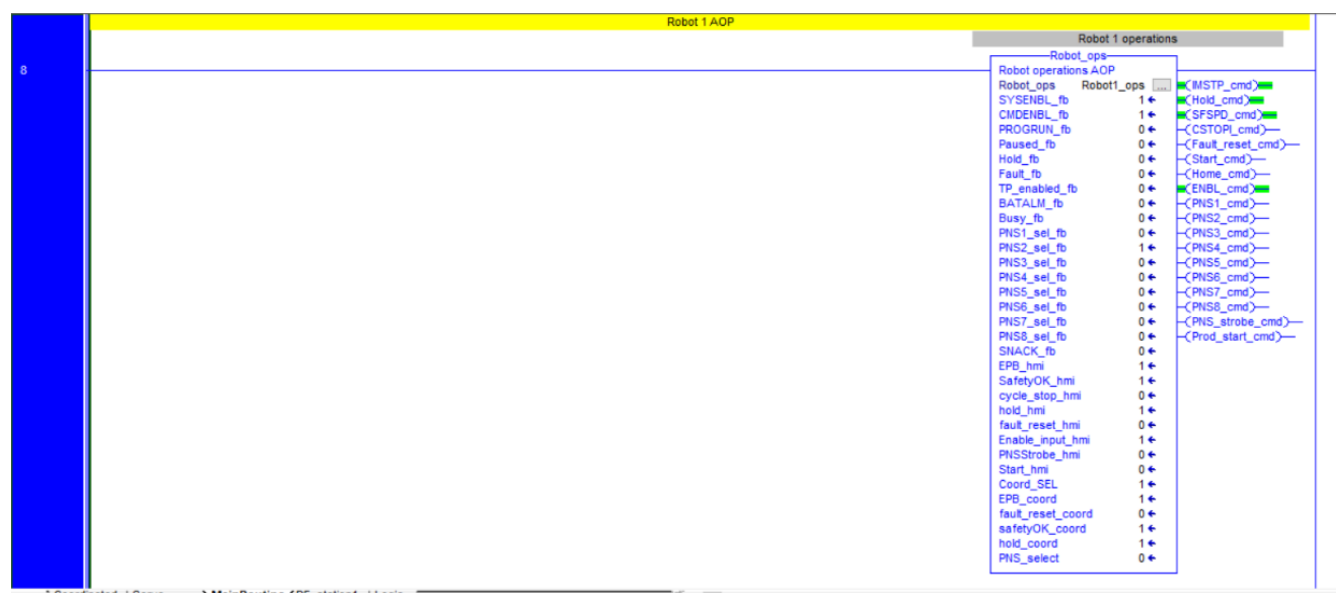

Figure 56 Robot_ops Add-On-instruction called in the program for Robot 1 
The Robot_ops AOI controls the User Operator Panel inputs on the robots to control the selection and execution of programs to follow the algorithm shown in Table 2.

Table 2 Robot UOP input functions

\begin{tabular}{|c|c|c|c|}
\hline UOP input & Input description & Input function & $\begin{array}{l}\text { Data } \\
\text { type }\end{array}$ \\
\hline UI[1] IMSTP & $\begin{array}{l}\text { Emergency stop } \\
\text { signal }\end{array}$ & $\begin{array}{l}\text { When OFF cuts power to } \\
\text { servos }\end{array}$ & Boolean \\
\hline UI[2] Hold & $\begin{array}{l}\text { Program hold } \\
\text { command }\end{array}$ & $\begin{array}{l}\text { When OFF slow motion to } \\
\text { controlled stopping a robot }\end{array}$ & Boolean \\
\hline UI[3] SFSPD & Safety signal & $\begin{array}{c}\text { When OFF inhibits a cycle } \\
\text { start }\end{array}$ & Boolean \\
\hline UI[4] CSTOPI & Cycle stop input & $\begin{array}{l}\text { Aborts current program and } \\
\text { clears program queue }\end{array}$ & Boolean \\
\hline UI[5] FAULT_RESET & $\begin{array}{c}\text { Fault reset } \\
\text { command for the } \\
\text { robots }\end{array}$ & Clearing robot error status & Boolean \\
\hline UI[6] START & Start command & $\begin{array}{l}\text { Resume a paused program } \\
\text { or start from cursor position }\end{array}$ & Boolean \\
\hline UI[8] ENBL & $\begin{array}{l}\text { Robot enable } \\
\text { command }\end{array}$ & Enable control of the robot & Boolean \\
\hline $\begin{array}{l}\mathrm{UI}[9]-\mathrm{UI}[16] \\
\text { PNS000xx }\end{array}$ & $\begin{array}{c}\text { Program number } \\
\text { select }\end{array}$ & $\begin{array}{c}\text { Selection of program } \\
\text { number to execute }\end{array}$ & SINT \\
\hline UI[17]PNSTROBE & $\begin{array}{l}\text { Program number } \\
\text { select strobe }\end{array}$ & $\begin{array}{l}\text { Two-second pulse signal to } \\
\text { conform program selection }\end{array}$ & Boolean \\
\hline UI[18]PROD_START & $\begin{array}{l}\text { Production start } \\
\text { command }\end{array}$ & $\begin{array}{c}\text { Used with PNSSTROBE } \\
\text { and executes selected } \\
\text { program }\end{array}$ & Boolean \\
\hline
\end{tabular}

The servo conveyor uses AOIs shown in Table 3 to control the servo conveyor and is downloaded from the Applied Motion website. The master PLC program writes commands to the AOIs from Table 3 to control the servo conveyor.

Table 3 Servo conveyor Add-On-Instructions

\begin{tabular}{cc}
\hline AOI name & AOI function \\
\hline AMP_Alarm_Code & Fetching servo alarm codes from the servo \\
\hline AMP_Alarm_Reset & Resetting alarm and clearing alarm codes on the servo \\
\hline AMP_Input_Assembly & Fetching Voltage, Current and Encoder values on the PLC \\
\hline AMP_Jog_Move & Transmit jog commands from the PLC to the servo conveyor \\
\hline AMP_Motor_Enable & Motor enable command from the PLC \\
\hline AMP_Normal_Stop & Stopping the jog of the conveyor \\
\hline AMP_Status_Code & Fetching status codes from the servo to PLC \\
\hline
\end{tabular}


The Donald Engineering station uses the DE_stn AOI to control the actuators when the station is in remote mode. The AOI is programmed to pass command inputs from the HMI to the Unitronics PLC on the Donald Engineering station shown in Figure 57.

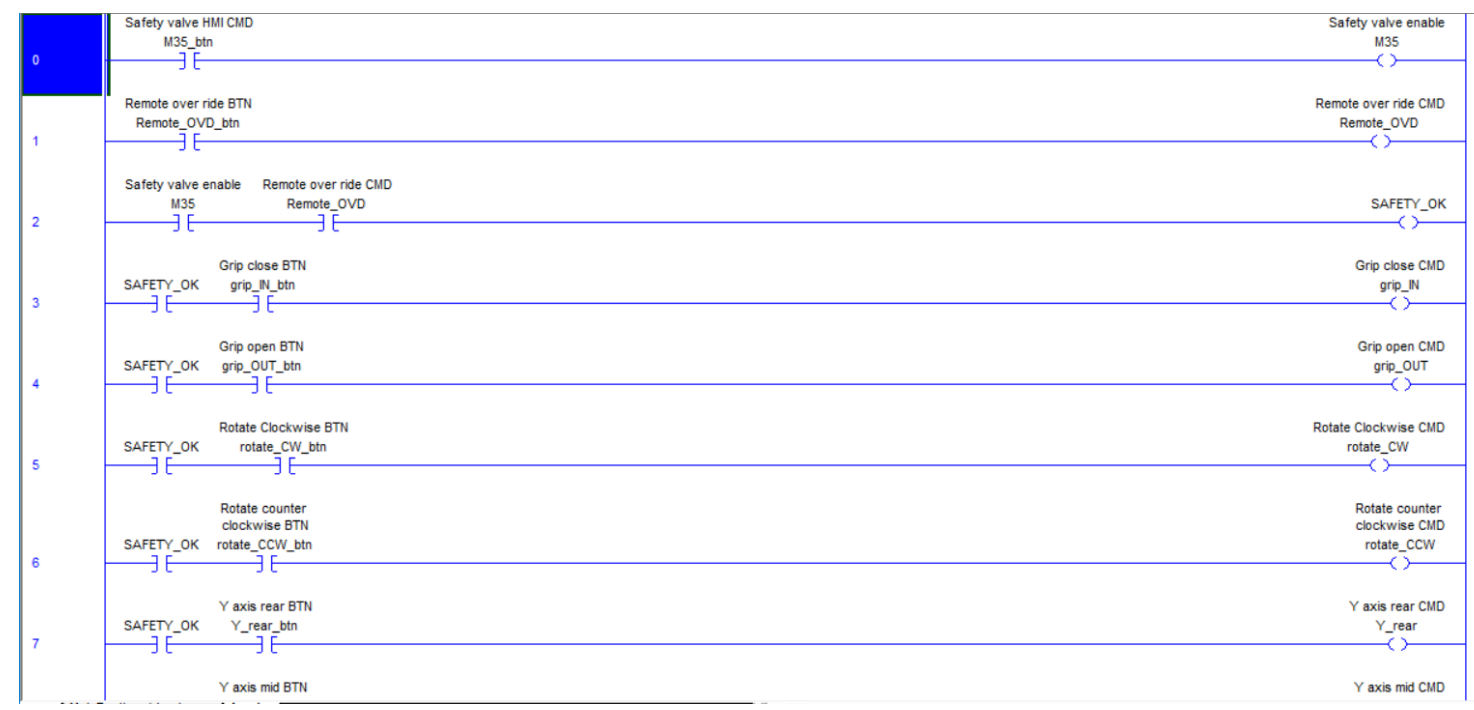

Figure 57 DE Station Add-On-Instruction

When working as a part of the machining process, the Donald Engineering stations follow the algorithm from Figure 58 to pick and place the part on the chute.

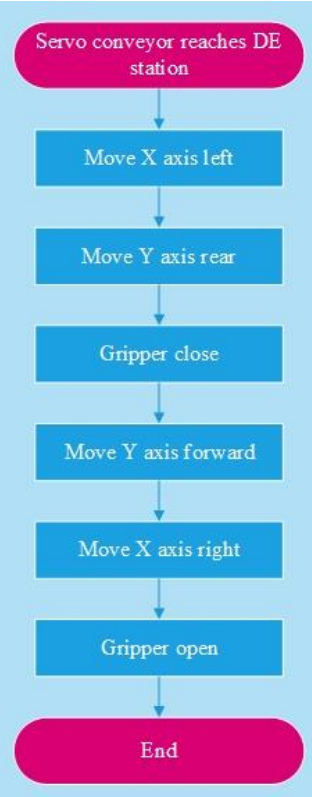

Figure 58 Algorithm for Donald Engineering station machining operations 


\section{Human Machine Interface}

The Human Machine Interface (HMI) is the medium through which a person can interact with the system. An HMI is deployable on any screen or computer and communicates with the controller providing inputs from the operator.

In the proposed system, the HMI is deployed on the Laptop using Rockwell Automation FactoryTalk View Studio Machine Edition software shown in Figure 59. It communicates with the master PLC over EthernetIP to control the process and subsystems.

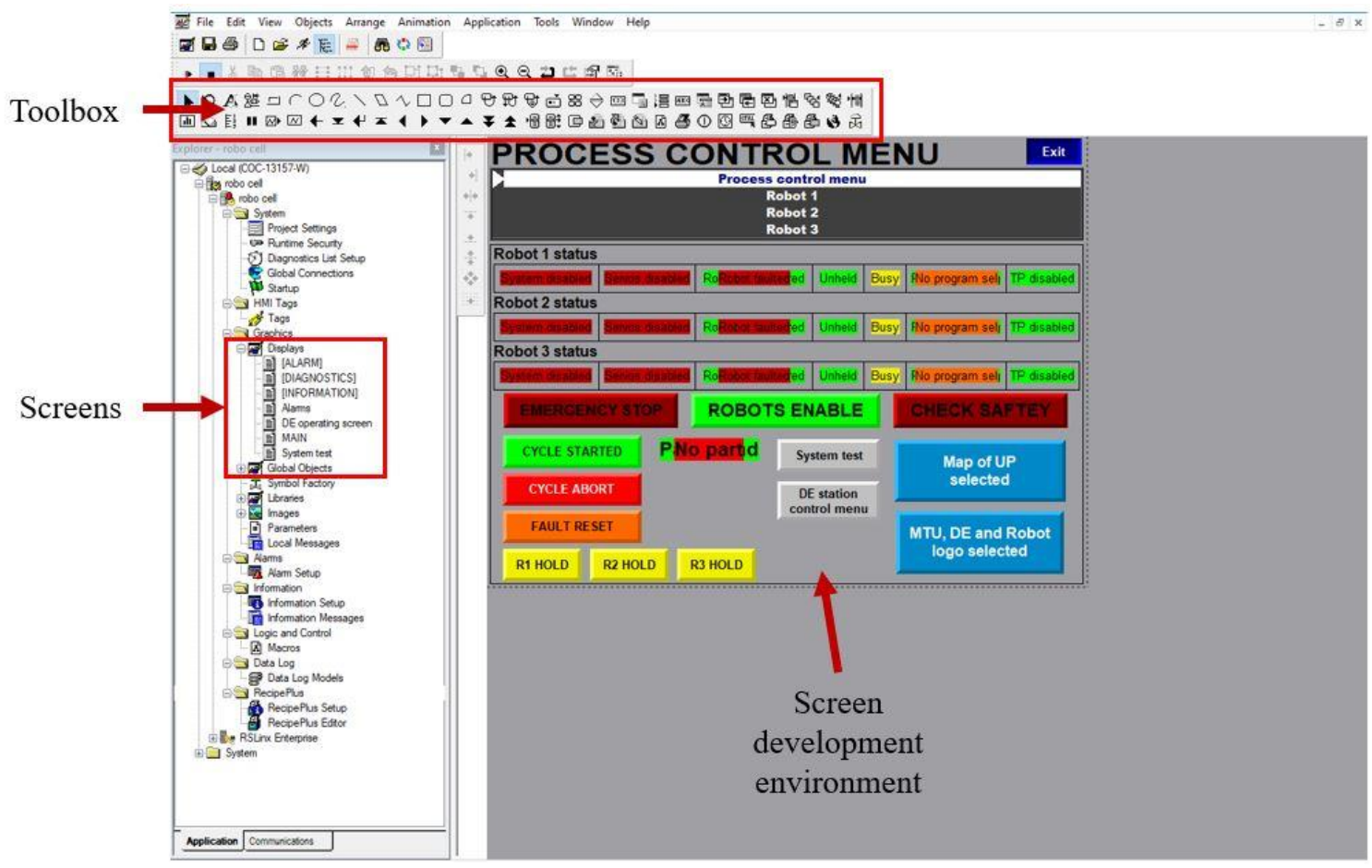

Figure 59 FactoryTalk View Studio interface

The software gives the programmer an option to create multiple screens with graphical user interfaces on the screen development environment. Toolbox objects as flashing indicators, pushbuttons, numeric entry panels, and selection lists provide control and status information to the operator.

\subsection{Robot control interface}

HMI interface allows the operator to control a single robot or run the entire machining process with all subsystems. 


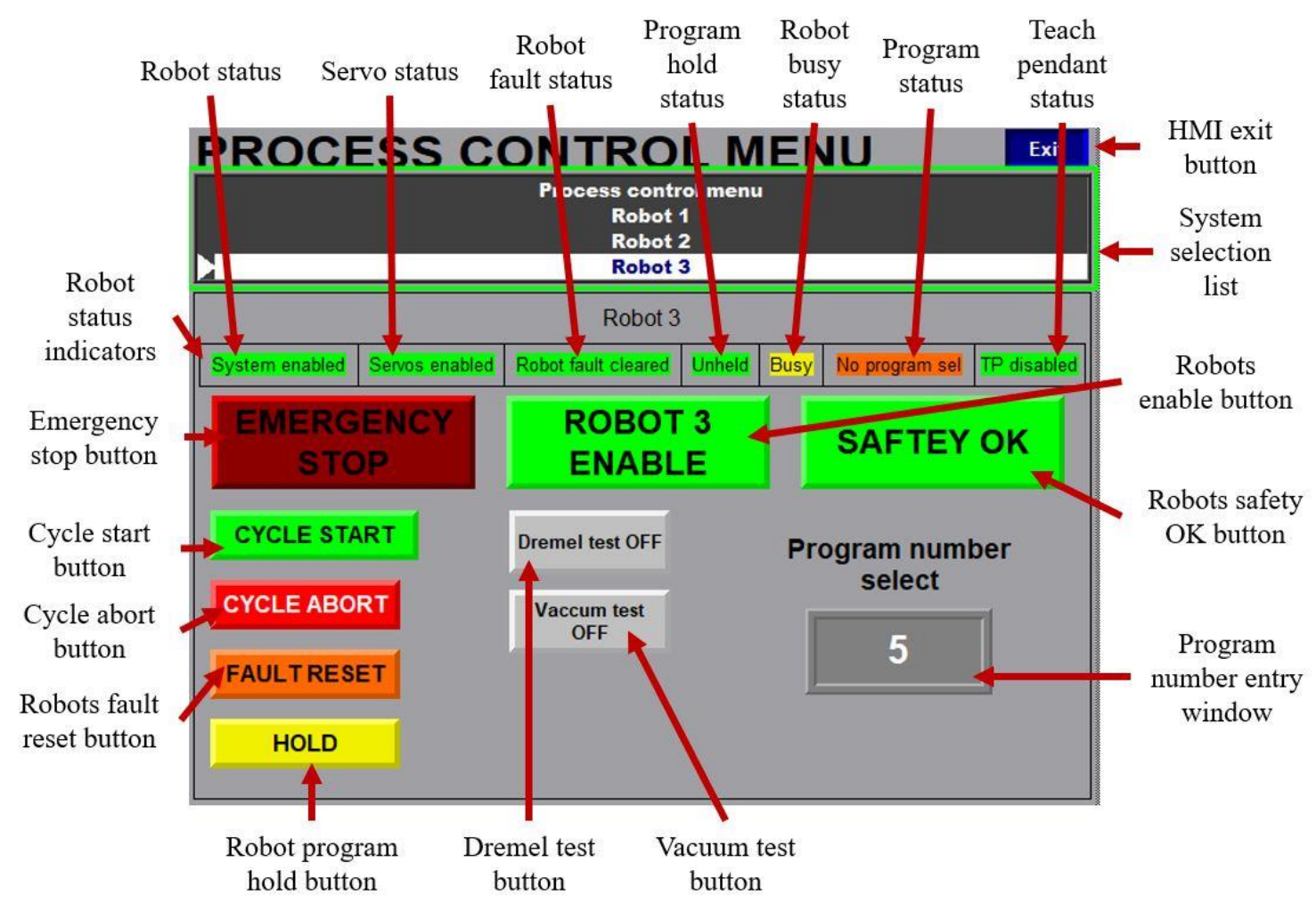

Figure 60 Robot control interface

The operator selects the program number, start, abort, hold the program and reset faults on the robot through the robot control interface shown in Figure 60. The operator unlatches the emergency stop, enables the robot and safety OK buttons before executing the selected program. The robot status indicators show various robot states, as shown in Figure 60. The Dremel and vacuum test buttons are exclusive to Robot 3 and are used to test their operation before starting a program.

\subsection{System test menu}

All systems are individually tested to avoid disruptions during the machining process and ensure correct functionality. For example, testing of the Dremel tooling, Vacuum, the belt conveyor, and the servo conveyor is executed from the system test menu shown in Figure 61. 


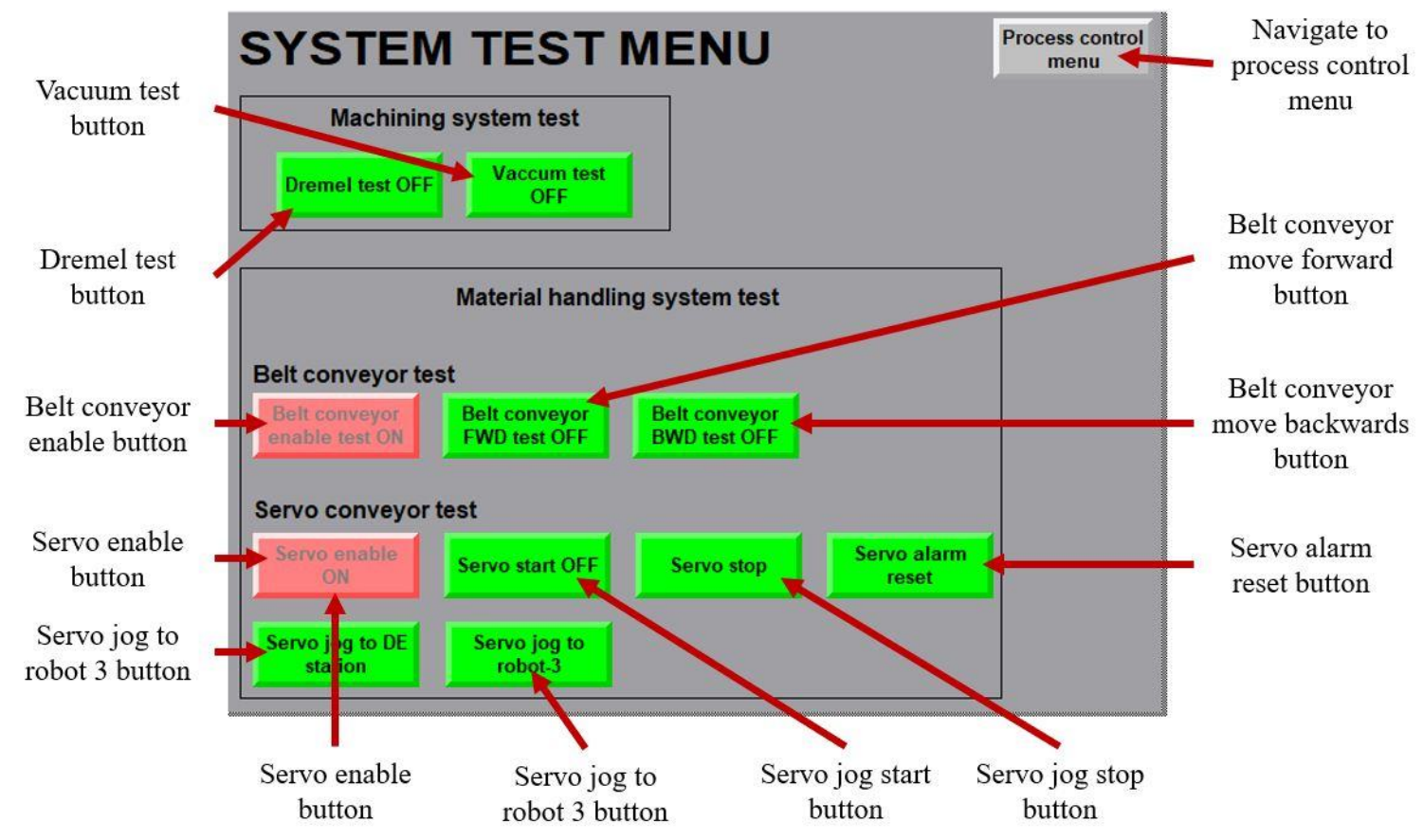

Figure 61 System test menu

\subsection{Donald Engineering station control menu}

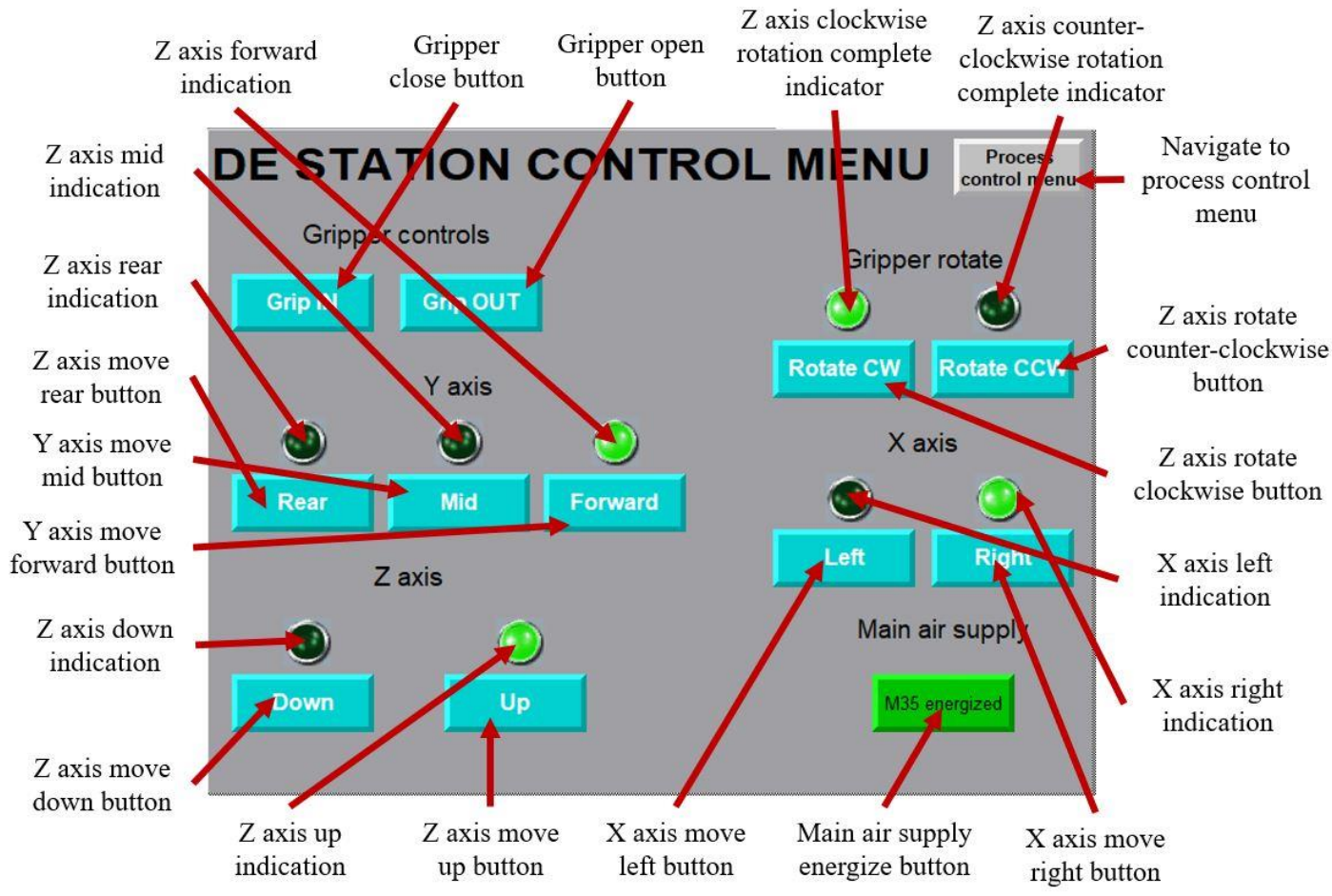

Figure 62 Donald Engineering station control menu 
The Donald Engineering station is operated from the master PLC when in remote mode as a part of the machining process and using the Unitronics HMI when in local mode.

The station can also be operated from the master PLC from the Donald Engineering station control menu shown in Figure 62 to test each actuator's functionality and serves as a backup control interface if the Unitronics HMI fails.

\subsection{Process control menu}

The process control menu, shown in Figure 63, is the interface for controlling the machining process.

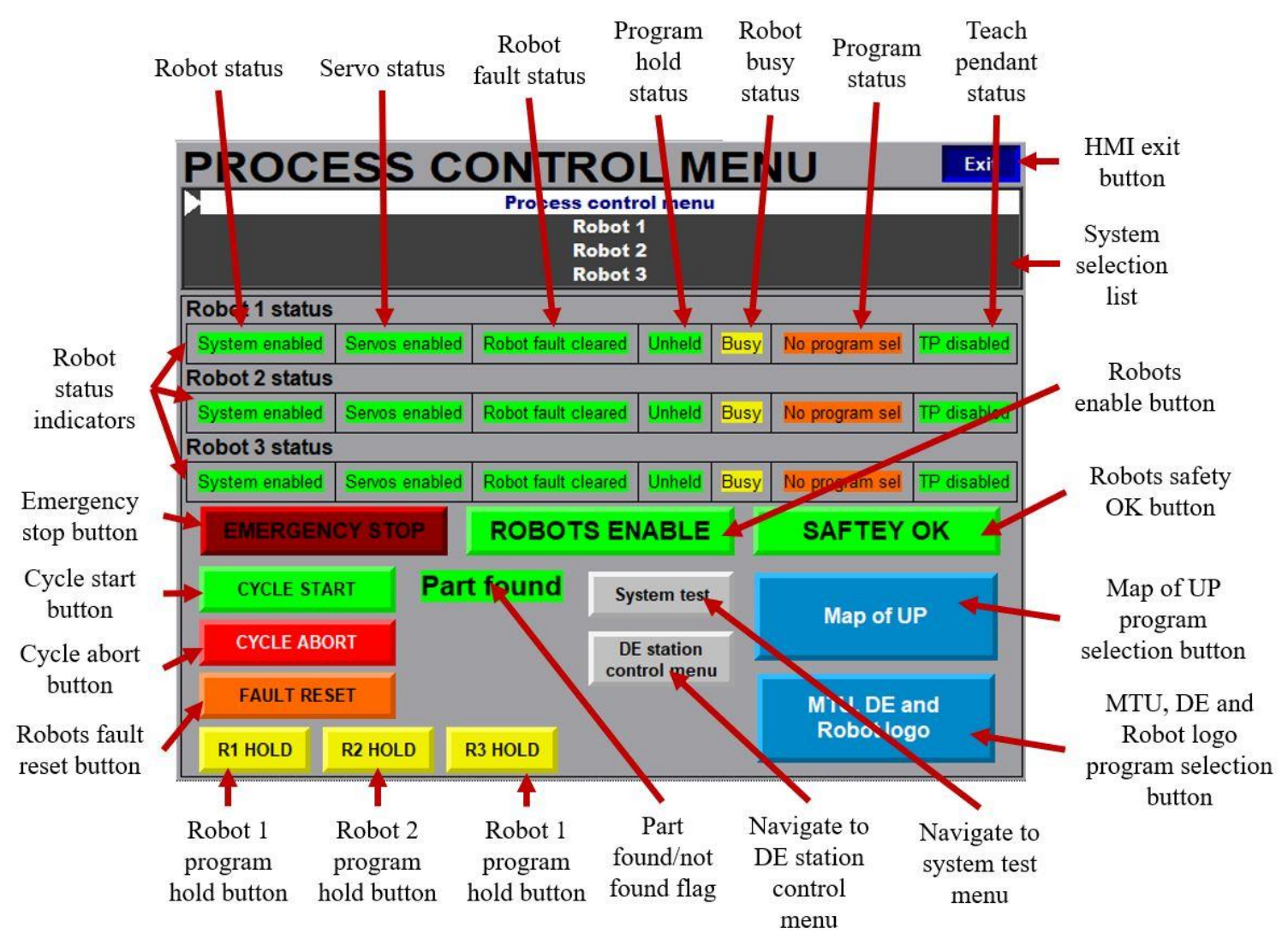

Figure 63 Proces control menu

The method for selecting, starting, aborting, holding a program, and resetting faults for the process control menu is similar to the robot control menu but is executed for all three robots simultaneously.

The robot status indicators show the current state of all three robots, and the "Part found" flag indicates if the workpiece is detected. The system test and DE station control menus are also accessed from the process control menu screen shown in Figure 63. 


\section{Final results}

Due to the complexity of the system and the number of components involved, the project achieved multiple results as follows:

\subsection{Achieving systems integration}

The final system results from the integration of multiple and different-task carrying subsystems into one fully functional process.

To integrate these fragmented components, the robots, Donald Engineering station, servo conveyor, and belt conveyor were configured to communicate over EthernetIP and hardwired connections with the overall goal to replicate the industrial machining operation. The robots were configured to use quick tool changers to increase the flexibility and reconfigurability of the processes. A master PLC and an HMI interface were designed and integrated into the system to provide a central control function.

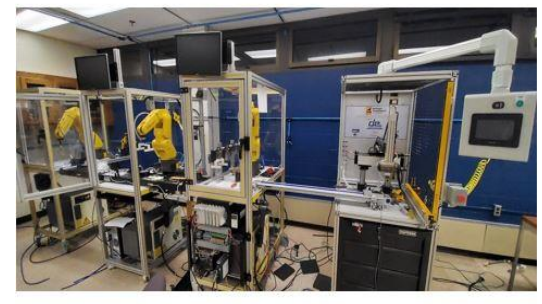

Overall system
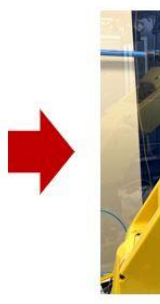

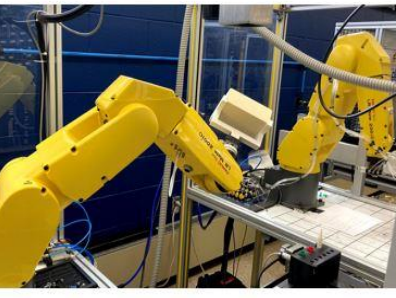

Subsystem (ex machining subsystem)
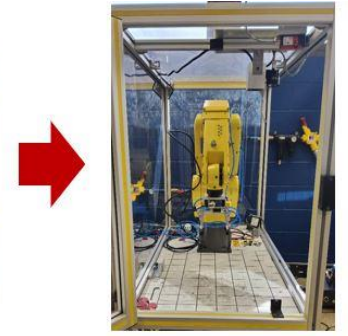

Component (ex Robot 2 training cart)

Figure 64 Final system integration process

The capability of an interconnected system has been demonstrated by a process developed to inspect and machine a workpiece involving all the components. If the overall system for the process is assumed from a top-down approach, then the final system consists of interconnected subsystems, and the subsystems are comprised of various components carrying specific functions. This top-down approach is demonstrated in Figure 64.

\subsection{Perfecting a communication architecture}

The communication between subsystems is an essential part of systems integration. The system uses modern communication protocols EthernetIP, enabling larger data sizes to be transferred at high speeds.

Connecting the subsystems in a star topology through an unmanaged Ethernet switch allows for simpler network architecture and ease in future expansion. The Ethernet-based communications on the robots and the PLCs unlock potential applications related to data acquisition and analytics. 


\subsection{Standardization and reconfigurability}

In the final system, the master PLC acts as a master controller for the robots, Donald Engineering station, and the servo conveyor utilizing the control algorithms deployed using the developed Add-On-Instructions. The benefit of programing with Add-On-Instructions is creating a block of code that can be used repeatedly in any system having a similar control algorithm leading to standardization. This enables the programmer to quickly deploy control algorithms for similar systems and create control interfaces that reduce operator training time.

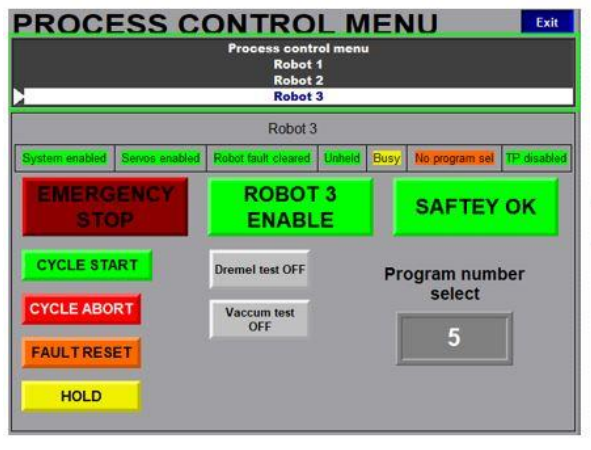

HMI screens

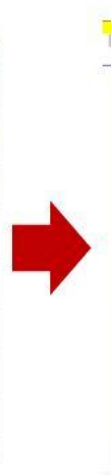

Robot Add-On-Instruction

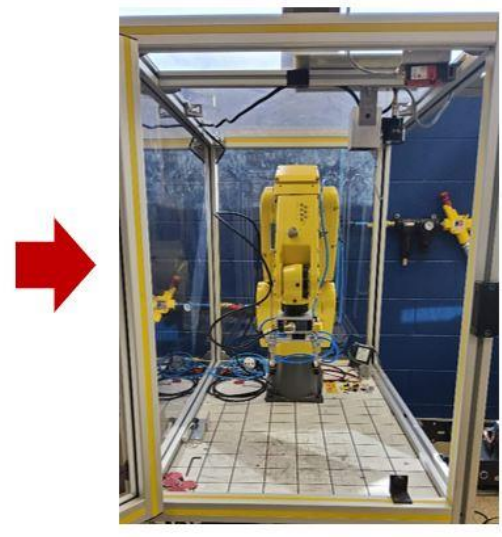

Robot

Figure 65 Standardization of robot control interface and algorithm

In addition to mentioned above benefits, the standardization of control interfaces and algorithms leads to reconfigurability in the system. The existing system can be broken down into components and assembled into a new system as a part of any future projects while utilizing previously developed HMI screens and Add-On-Instructions to control the subsystems.

\subsection{Validating the accuracy of robotic machining}

The inspection and machining process outputs a machined workpiece having a repeatable and accurate machining pattern. The FANUC robots used to machine the workpiece are highly repeatable while maintaining the accuracy in microns, ensuring that the next produced workpiece is a replica of the previously made workpiece.

The accuracy of the production process is validated by the cross-correlation function implemented in Matlab. The correlation program uses four machined workpieces photographed in grayscale, shown in Figure 67, and correlates its features utilizing the algorithm demonstrated in Figure 66. The images are lined up using holes drilled by the robot on four recorded positions. The output of the $2 \mathrm{D}$ correlation ranges from -1.0 to 1.0, with 1.0 being a perfect match and -1.0 perfectly inverted image. 


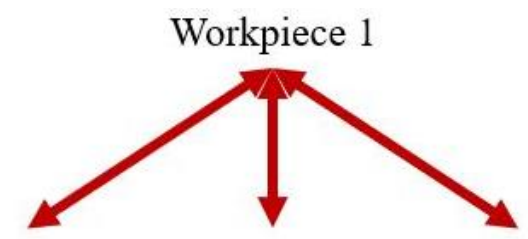

Workpiece 2 Workpiece 3 Workpiece 4

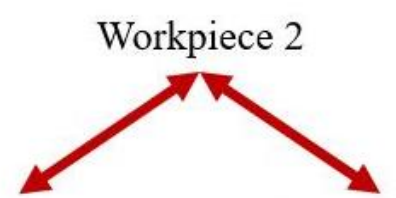

Workpiece 3

Workpiece 4

Figure 66 Correlation program algorithm
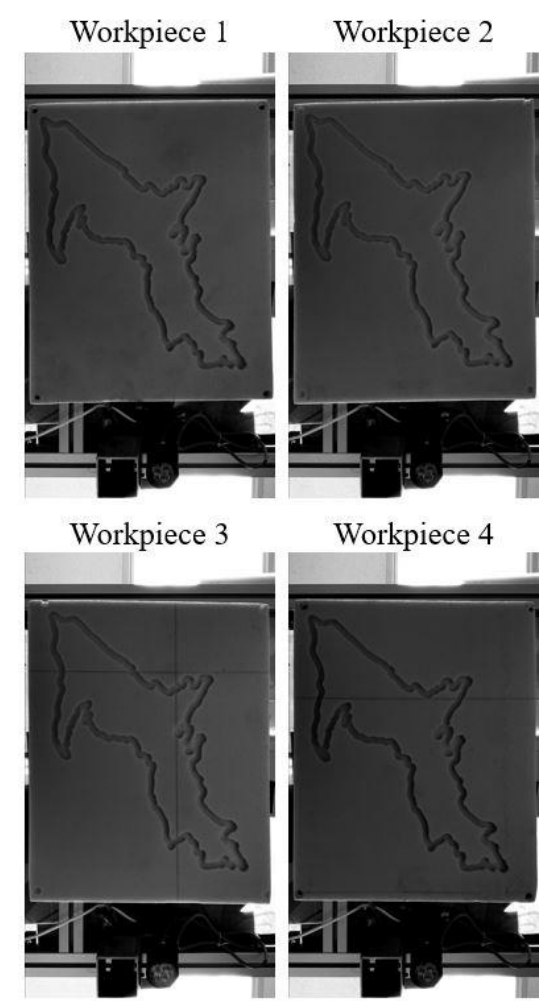

Figure 67 Machined workpieces

Figure 68 shows the correlation program and the program results for the machined workpieces. The result shows the correlation values ranging from 0.9559 to 0.9806 , indicating the machined pattern's accuracy and repeatability. The error can be accounted towards minor dimensional inaccuracies of the raw workpiece and vibrations due to the wheel-mounted FANUC training cart. 


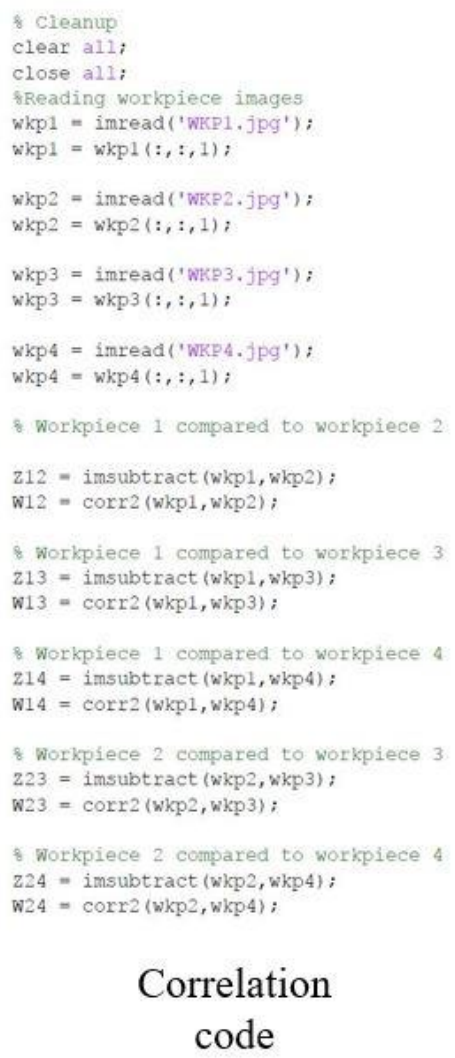

Correlation code

MATLAB Workspace

Mar 29, 2021

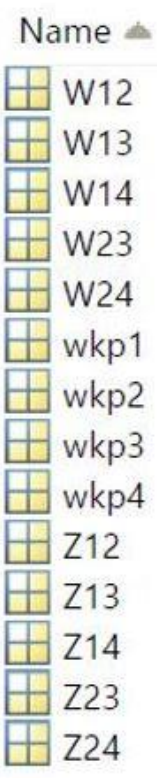

Correlation
Value

0.9616

0.9564

0.9559

0.9706

0.9806

$3088 \times 1737$ uint8

$3088 \times 1737$ uint 8

$3088 \times 1737$ uint8

$3088 \times 1737$ uint8

$3088 \times 1737$ uint8

$3088 \times 1737$ uint 8

$3088 \times 1737$ uint8

$3088 \times 1737$ uint8

$3088 \times 1737$ uint8

Figure 68 Correlation program results

\subsection{Educational impact}

The final system serves as a great example of various subsystems integration using different communication protocols, control algorithms, and hardware commonly used in the industry. The information generated from this project is being used to create educational materials and the lab exercises related but not limited to the following areas of educational competencies:

1. Configuration of EthernetIP communication on Rockwell Automation PLCs, FANUC robots, and Donald Engineering stations for data exchange

2. Design and integration of Add-On-Instructions to control program selection and execution on the robots

3. Design and control of quick tool changers for various applications

4. Design and control of vacuum grippers for different size and orientation of objects

5. Control and applications of electro-pneumatic robots

6. Motion control using variable speed drives and servos

7. Controlling material handling systems to transfer parts between stations 


\section{System Limitations}

The proposed project is an excellent example of systems integration with flexibility and reconfigurability designed into it, which is the primary goal of this research project. With the current hardware, tooling, and payload of the robots, the system is designed to work with soft materials such as low to medium density $\mathrm{CNC}$ foam for machining applications. Higher density materials may require redesigning some of the 3D printed end-effectors and tool brackets. The accuracy and repeatability of the machined workpieces are evaluated using a cross-correlation function implemented in MATLAB. With the current settings and hardware used, the results demonstrated high accuracy and repeatability of the produced parts. However, the use of higher density materials may require using more sturdy mounts and end-effectors for compensating additional torque induced by the Dremel tooling. One solution to accounting for different densities of the used materials is to clamp the workpiece to a fixture instead of being held on a robot. Also, more data needs to be collected to fully understand the impact of using the materials with different densities on the produced results. 


\section{Reference List}

[1] J. Antony, B. Mahato, S. Sharma and G. Chitranshi, "A Web PLC Using Distributed Web Servers for Data Acquisition and Control: Wed Based PLC," 2011 International Conference on Information Science and Applications, Jeju Island, 2011, pp. 1-4, doi: 10.1109/ICISA.2011.5772427.

[2] H. S. Jeong, S. H. Ji, H. S. Jung and J. C. Koo, "Design of SW architecture for PLC integrated robot," 2017 14th International Conference on Ubiquitous Robots and Ambient Intelligence (URAI), Jeju, 2017, pp. 874-876, doi: 10.1109/URAI.2017.7992851.

[3] B. A. Brandin, "The real-time supervisory control of an experimental manufacturing cell," in IEEE Transactions on Robotics and Automation, vol. 12, no. 1, pp. 1-14, Feb. 1996, doi: 10.1109/70.481746.

[4] B. B. Malakanagouda and S. K. Harisha, "PLC based automation of test rig for brake actuators," 2017 International Conference on Energy, Communication, Data Analytics and Soft Computing (ICECDS), Chennai, 2017, pp. 1238-1243, doi: 10.1109/ICECDS.2017.8389639.

[5] T. Kubela, A. Pochyly and V. Singule, "Assessment of industrial robots accuracy in relation to accuracy improvement in machining processes," 2016 IEEE International Power Electronics and Motion Control Conference (PEMC), Varna, 2016, pp. 720-725, doi: 10.1109/EPEPEMC.2016.7752083.

[6] J. Lee, G. Park, J. Shin and J. Woo, "Industrial robot calibration method using denavit - Hatenberg parameters," 2017 17th International Conference on Control, Automation and Systems (ICCAS), Jeju, 2017, pp. 1834-1837, doi: 10.23919/ICCAS.2017.8204265.

[7] M. S. Abderrahmane, A. M. Djuric, W. Chen and C. P. Yeh, "Study and validation of singularities for a Fanuc LR Mate 200iC robot," IEEE International Conference on Electro/Information Technology, Milwaukee, WI, 2014, pp. 432-437, doi: 10.1109/EIT.2014.6871803.

[8] Chan Woo Moon, Beom Hee Lee and M. S. Kim, "PLC based coordination schemes for a multi-robot system," Proceedings 2001 ICRA. IEEE International Conference on Robotics and Automation (Cat. No.01CH37164), Seoul, South Korea, 2001, pp. 3109-3114 vol.3, doi: 10.1109/ROBOT.2001.933095.

[9] L. Bassi, "Industry 4.0: Hope, hype or revolution?," 2017 IEEE 3rd International Forum on Research and Technologies for Society and Industry (RTSI), Modena, 2017, pp. 1-6, doi: 10.1109/RTSI.2017.8065927.

[10] Unitronics PLC communication configuration for Rockwell Automation PLCs https://support.unitronics.com/index.php?/selfhelp/view-article/EIP-with-Allen-Bradley 
[11] Quick tool changer manifold wiring diagram - https://clippard.com/part/EMC-08-24$\underline{20}$

[12] Photoelectric sensor wiring diagram https://www.automationdirect.com/adc/shopping/catalog/sensors _-z_encoders/photoelectric_sensors/18mm_round___nonmetal/through_beam_(ss_z-_fa_-zfb_series)/ssr-0p-4a

[13] Servo conveyor driver AOIs and wiring data - https://www.appliedmotion.com/products/servo-drives/sv2a3-ip-de

[14] Belt conveyor driver manual https://www.orientalmotor.com/products/pdfs/opmanuals/HM-5171-3E.pdf

[15] FANUC R30iA programming manual

[16] FANUC LRMate 200iC operating manual

[17] FANUC 2DiR Vision manual 


\section{A Appendix}

\section{A.1 Vision system hardware details}

Table A.1. Vision system hardware specifications

\begin{tabular}{|c|c|c|}
\hline $\begin{array}{l}\text { Sr. } \\
\text { No. }\end{array}$ & Name & Description \\
\hline 1 & Camera & $\begin{array}{l}\text { SONY XC-56 } \\
\text { Black \& white CCD camera, } 659 \text { X } 494 \text { pixel array } \\
\text { running at } 30 \text { frames/sec, VGA resolution }\end{array}$ \\
\hline 2 & Spotlight & $\begin{array}{l}\text { Model LEDWS50L20-XQ } \\
\text { High intensity LED White spotlight, } 3 \text { LED's }\end{array}$ \\
\hline
\end{tabular}

\section{A.2 Donald Engineering station PLC hardware details}

Table A.2. Donald Engineering station PLC hardware specifications

\begin{tabular}{lll}
\hline $\begin{array}{c}\text { Sr. } \\
\text { No. }\end{array}$ & \multicolumn{1}{c}{ Name } & \multicolumn{1}{c}{ Description } \\
\hline 1 & USC-B5-B1 & Unitronics UniStream series PLC \\
& Qty - 1 & $\begin{array}{l}\text { 1MB storage, 24VDC power supply, 2 } \\
\text { Ethernet ports }\end{array}$ \\
\hline 2 & UID- & Unitronics Uni-I/O \\
& W1616R & $\begin{array}{l}\text { 16 24VDC isolated inputs, 16 isolated relay } \\
\text { outputs }\end{array}$ \\
\hline 3 & Qty - 1 & Unitronics Uni-I/O \\
& Qty - 1 & 16 24VDC isolated inputs \\
\hline 4 & UID-0016R & Unitronics Uni-I/O \\
& Qty - 1 & 16 isolated relay outputs \\
\hline
\end{tabular}




\section{A.3 Master PLC hardware details}

Table A.3. Master PLC hardware specifications

\begin{tabular}{cll}
\hline $\begin{array}{c}\text { Sr. } \\
\text { No. }\end{array}$ & Name & \multicolumn{1}{c}{ Description } \\
\hline 1 & 1769 L32E & Allen Bradley CompactLogix series controller \\
& Qty - 1 & $\begin{array}{l}\text { 750KB storage, 1 port each for EthernetIP, } \\
\text { RS232 }\end{array}$ \\
\hline 2 & 1769-PA2 & $\begin{array}{l}\text { Allen Bradley 1769 series compact power } \\
\text { supply }\end{array}$ \\
& Qty - 1 & Selectable 120/220V, 2A, 85-256VAC input \\
\hline 3 & $1769-$ IQ16 & Allen Bradley 1769 series compact IO's \\
& Qty - 1 & 16 point sink/source 24VDC input module \\
\hline 4 & $1769-$ & Allen Bradley 1769 series compact IO's \\
& OB16 & 16 point discrete 24VDC output module \\
& Qty - 3 & \\
\end{tabular}

\section{A.4 Robot 2 end of the arm tooling details}

Table A.4. Robot 2 end of the arm tooling gripper specifications

\begin{tabular}{cll}
\hline $\begin{array}{c}\text { Sr. } \\
\text { No. }\end{array}$ & Name & \multicolumn{1}{c}{ Description } \\
\hline 1 & PGN- & Schunk PGN-40 \\
& 40 & 6 Bar operating pressure, 6mm stroke per jaw, \\
& Qty - 2 & 110N opening force \\
\hline
\end{tabular}




\section{A.5 Quick tool changer hardware details}

Table A.5. Quick tool changer hardware specifications

\begin{tabular}{|c|c|c|}
\hline $\begin{array}{l}\text { Sr. } \\
\text { No. }\end{array}$ & Name & Description \\
\hline \multirow[t]{3}{*}{1} & PGN Plus 64- & Schunk PGN plus size 64 \\
\hline & & 6 Bar operating pressure, $6 \mathrm{~mm}$ stroke per jaw, \\
\hline & Qty - 4 & $360 \mathrm{~N}$ opening force \\
\hline \multirow[t]{2}{*}{2} & $\begin{array}{l}\text { SWA-011- } \\
000-000\end{array}$ & $\begin{array}{l}\text { Schunk SWA quick-change system with ZK } \\
\text { master and ZA adapters }\end{array}$ \\
\hline & Qty - 4 & $\begin{array}{l}6 \text { pneumatic feed through's, } 16 \mathrm{Kg} \text { handling } \\
\text { weight }\end{array}$ \\
\hline \multirow[t]{3}{*}{3} & $\begin{array}{l}\text { SWO-EM8- } \\
011-A\end{array}$ & \\
\hline & & 8 pin feedthrough, $1.5 \mathrm{~A}$ nominal current \\
\hline & Qty - 4 & \\
\hline \multirow[t]{2}{*}{4} & SWS-S & Schunk modular storage magazine \\
\hline & Qty - 4 & 3 alignment pins \\
\hline
\end{tabular}

\section{A.6 Dremel tooling specifications}

Table A.6. Dremel tooling hardware specifications

\begin{tabular}{lll}
\hline $\begin{array}{c}\text { Sr. } \\
\text { No. }\end{array}$ & Name & \multicolumn{1}{c}{ Description } \\
\hline 1 & Dremel & Dremel 4000-4/34 series. \\
& & 120V corded single-phase supply, 5000-35,000 rpm \\
& & selectable speed \\
\hline 2 & Flex & $\begin{array}{l}\text { Dremel 225-01 rotary attachment.36" length, 1/8" } \\
\text { shank, 2 flute 1/8" HSS drill bit }\end{array}$ \\
& &
\end{tabular}




\section{A.7 Belt conveyor hardware details}

Table A.7. Belt conveyor specifications

\begin{tabular}{lll}
\hline $\begin{array}{c}\text { Sr. } \\
\text { No. }\end{array}$ & Name & \multicolumn{1}{c}{ Description } \\
\hline 1 & Conveyor & $\begin{array}{l}\text { Mini Mover Conveyors, LITE series model } \\
\text { Width }-10 ", \text { Length }-84 ”, \text { Speed 3-43 } \\
\text { feet/min }\end{array}$ \\
\hline 2 & $\begin{array}{l}\text { Conveyor } \\
\text { frame }\end{array}$ & $\begin{array}{l}\text { Hard black anodized aluminum frame with } \\
\text { integral .70" high side guides }\end{array}$ \\
\hline 3 & Belt & $\begin{array}{l}\text { Black, PVC (polyvinyl chloride, low friction } \\
\text { cover) }\end{array}$ \\
\hline 4 & Geared & $\begin{array}{l}\text { Oriental Motor Co. Ltd., Brushless DC Motor, } \\
\text { motor } \\
\text { 100-120 V, Output Power - 60 W W }\end{array}$ \\
\hline 5 & $\begin{array}{l}\text { Variable } \\
\text { Speed Drive }\end{array}$ & $\begin{array}{l}\text { Oriental Motor Co. Ltd., 115/60 VAC input } \\
\text { variable speed Digital Display, Model No. } \\
\text { BMUD60-A2 }\end{array}$ \\
& &
\end{tabular}

\section{A.8 Photoelectric sensors specifications}

Table A.8. Photoelectric sensor specifications

\begin{tabular}{lll}
\hline $\begin{array}{l}\text { Sr. } \\
\text { No. }\end{array}$ & \multicolumn{1}{c}{ Name } & \multicolumn{1}{c}{ Description } \\
\hline 1 & $\begin{array}{l}\text { Photoelectric sensor } \\
\text { receiver }\end{array}$ & Automation Direct SSR-0P-4A \\
& & $\begin{array}{l}24 \mathrm{~V} \text { DC, PNP type, Normally } \\
\text { Open operation. }\end{array}$ \\
\hline 2 & $\begin{array}{l}\text { Photoelectric sensor } \\
\text { emitter }\end{array}$ & Automation Direct SSE-00-4A \\
& & 24V DC, PNP type \\
\hline
\end{tabular}




\section{A.9 Servo conveyor hardware details}

Table A.9. Photoelectric sensor specifications

\begin{tabular}{cll}
\hline $\begin{array}{c}\text { Sr. } \\
\text { No. }\end{array}$ & \multicolumn{1}{c}{ Name } & \multicolumn{1}{c}{ Description } \\
\hline 1 & $\begin{array}{l}\text { Linear } \\
\text { actuator }\end{array}$ & Schunk Delta D90 ZRS \\
& & $\begin{array}{l}\text { 4000mm length, 800Nm max force, BL } \\
\text { series fastening element }\end{array}$ \\
\hline 2 & $\begin{array}{l}\text { Servo } \\
\text { driver }\end{array}$ & Applied motion SV2A3-IP-DE \\
\hline
\end{tabular}

\section{A.10 Inductive proximity sensor hardware details}

Table A.10. Inductive proximity sensor specifications

\begin{tabular}{lll}
\hline \multicolumn{1}{c}{$\begin{array}{c}\text { Sr. } \\
\text { No. }\end{array}$} & \multicolumn{1}{c}{ Name } & \multicolumn{1}{c}{ Description } \\
\hline 1 & Inductive proximity & Kissling L408 \\
& switch & PNP switching, 3 wire 24V \\
& & DC 0.2A \\
\hline
\end{tabular}

